Aus der Abteilung Unfallchirurgie, Plastische und Wiederherstellungschirurgie (Prof. Dr. med. K. M. Stürmer) im Zentrum Chirurgie der Medizinischen Fakultät der Universität Göttingen

\title{
Die Arthrodese an den Sprunggelenken
}

\author{
INAUGURAL - DISSERTATION \\ zur Erlangung des Doktorgrades \\ der Medizinischen Fakultät \\ der Georg-August-Universität zu Göttingen
}

vorgelegt von

Jan-Michael Anger

aus

Lübeck

Göttingen 2010 
Dekan: Prof. Dr. med. C. Frömmel

I. Berichterstatter: Prof. Dr. med. K. Dresing

II. Berichterstatterlin:

III. Berichterstatter/in:

Tag der mündlichen Prüfung: 


\section{Inhaltsverzeichnis}

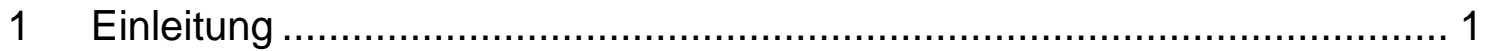

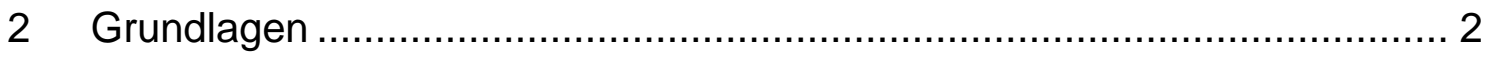

2.1 Topographische und funktionelle Anatomie der Sprunggelenke ........... 2

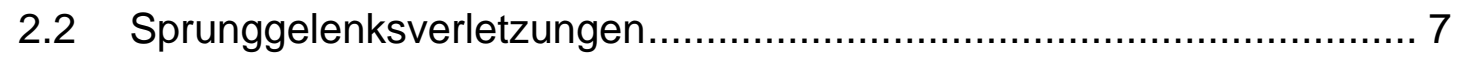

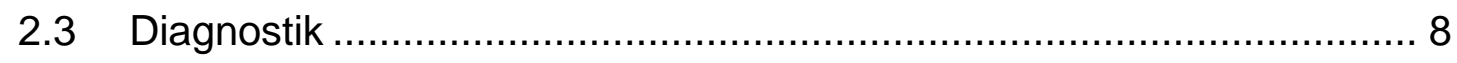

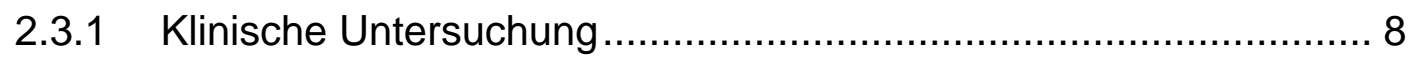

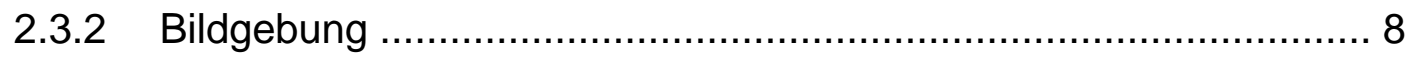

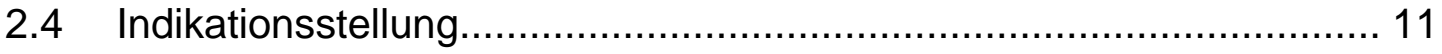

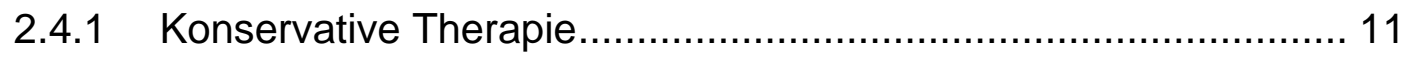

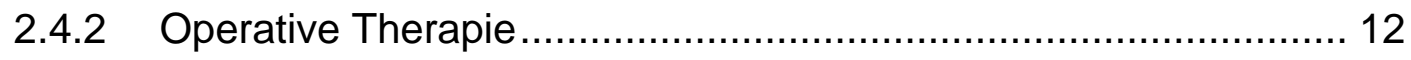

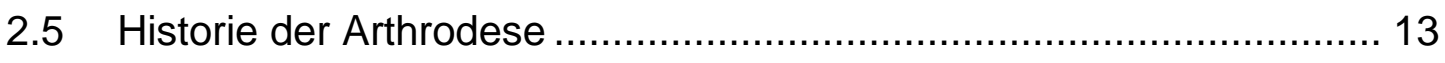

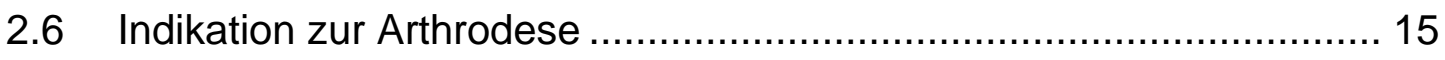

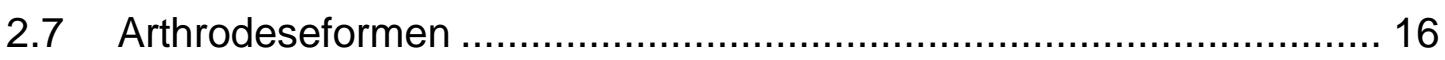

2.7.1 Die OSG-Arthrodese …..................................................... 17

2.7.2 Die USG-Arthrodese............................................................. 24

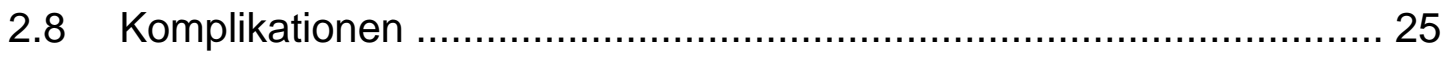

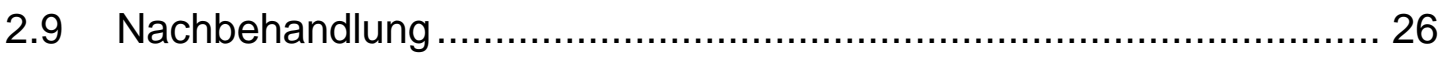

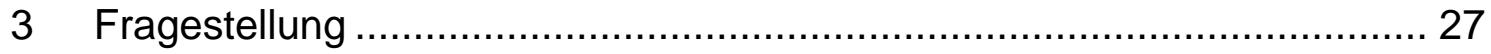

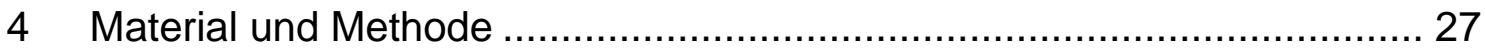

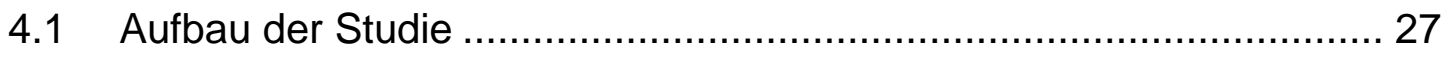

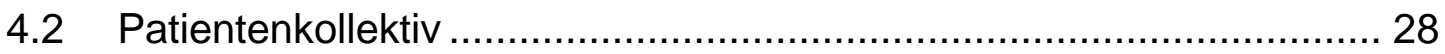

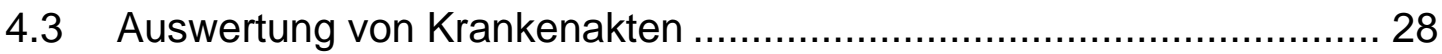

4.4 Erstellen und Versenden des Fragebogens .................................... 29

4.5 Klinische Untersuchung der Patienten ........................................... 30

4.6 Score 


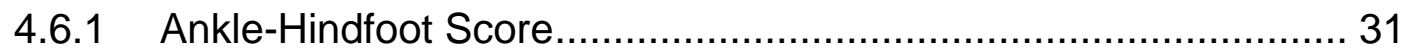

4.6.2 Mazur Score .............................................................. 33

4.6.3 McGuire et al. Score ...................................................... 33

4.7 Dokumentation, Statistik und Auswertung .................................. 34

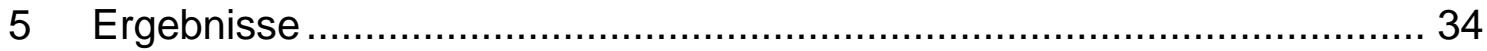

5.1 KDA versus Kompressionsarthrodese unabhängig vom Gelenk........ 34

$5.1 .1 \quad$ Altersverteilung .......................................................... 35

$5.1 .2 \quad$ Score-Ergebnisdarstellung .............................................. 36

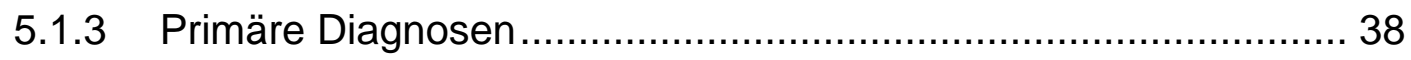

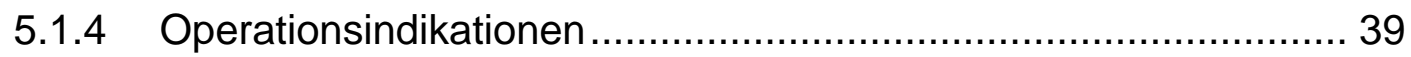

5.1.5 Perioperative Komplikationen und Spätfolgen ....................... 40

5.1.6 Dauerhafte Schmerzen nach OP ......................................... 42

5.1.7 Belastungsabhängige Schmerzen nach OP ........................ 43

5.1.8 Dauerhafte Schmerzmitteleinnahme nach OP ........................ 44

5.1.9 Schmerzfreie Gehstrecke nach OP .................................... 45

5.1.10 Subjektive Zufriedenheit........................................... 46

5.2 KDA am OSG versus KDA am USG …................................. 47

5.2.1 Darstellung des KDA-Kollektives ...................................... 47

5.2.2 Altersverteilung ............................................................ 48

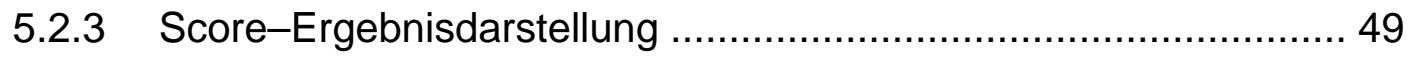

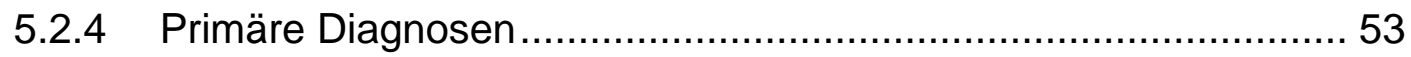

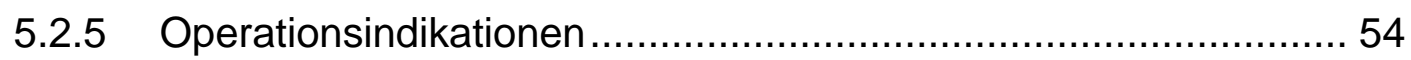

5.2.6 Perioperative Komplikationen und Spätfolgen......................... 55

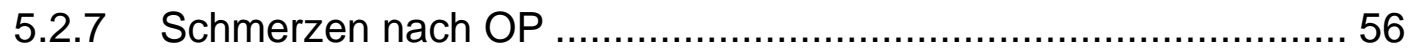

5.2.8 Belastungsabhängige Schmerzen nach OP .......................... 57

5.2.9 Dauerhafte Schmerzmitteleinnahme nach OP ........................ 58 
5.2.10 Schmerzfreie Gehstrecke nach OP .................................. 59

5.2.11 Subjektive Zufriedenheit............................................. 60

5.3 KDA am OSG versus Kompressionsarthrodese am OSG ................. 61

5.3.1 Darstellung des OSG-Kollektives ...................................... 61

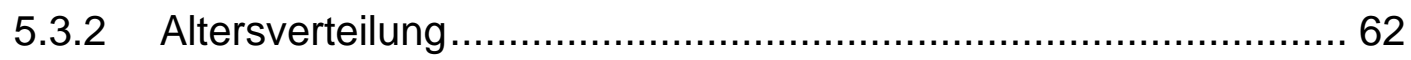

5.3.3 Score-Ergebnisdarstellung ............................................... 63

5.3.4 Primäre Diagnosen .................................................. 65

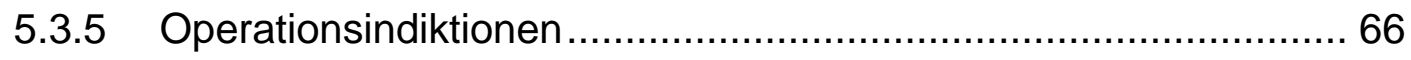

5.3.6 Perioperative Komplikationen und Spätfolgen......................... 67

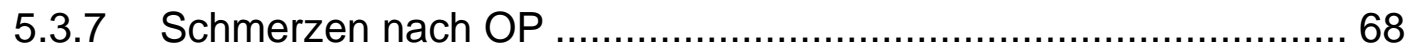

5.3.8 Belastungsabhängige Schmerzen nach OP ......................... 69

5.3.9 Dauerhafte Schmerzmitteleinnahme nach OP ....................... 70

5.3.10 Schmerzfreie Gehstrecke nach OP .................................. 71

5.3.11 Subjektive Zufriedenheit........................................... 72

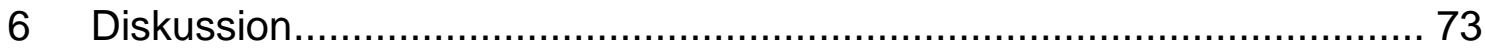

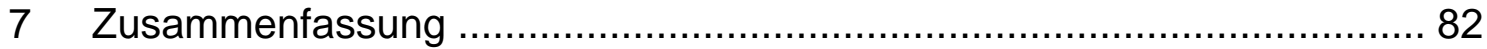

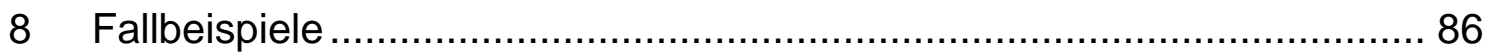

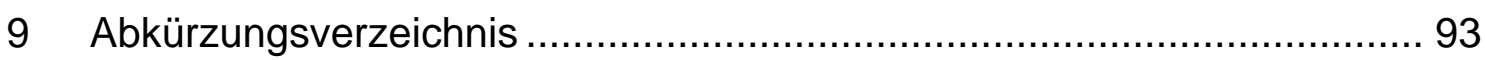

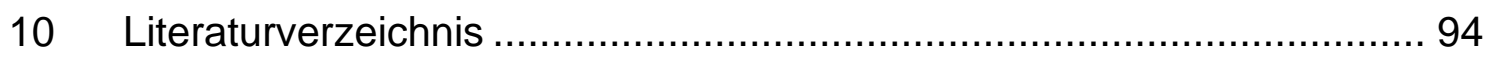

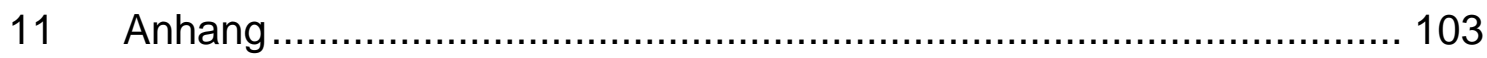

11.1 Traumatologische Klassifikationen ........................................ 103

11.1.1 Klassifikation nach Lauge-Hansen ............................... 103

11.1.2 Klassifikation nach Dennis Weber ................................. 109

11.1.3 AO-Klassifikation ................................................. 110

11.1.4 Klassifikation und Pathogenese der Kalkaneusfrakturen ........ 114

11.1.5 Klassifikation nach Sanders ................................... 115

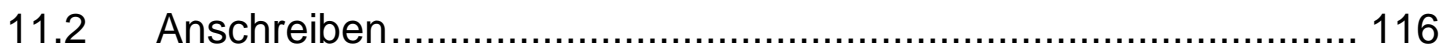


11.3 Patientenfragebogen ......................................................... 117

$11.4 \quad$ Patienteneinwilligung ................................................... 121 


\section{Einleitung}

In der Unfallchirurgie spielen Verletzungen der unteren Extremität, insbesondere des Unterschenkels, des oberen und unteren Sprunggelenks sowie des Fußes, eine wichtige Rolle.

In einem Zeitalter hoher beruflicher und privater Mobilität kommt es zu einem Ansteigen des Verkehrsaufkommens. Damit verbunden ist eine Zunahme von Unfällen mit einer entsprechenden Anzahl an Verletzten und Verletzungen der unteren Extremität.

Weitere Ursachen sind Unfälle in der Freizeit, im Haushalt und Arbeitsunfälle, die zur Einlieferung von Verletzten in unfallchirurgische Kliniken führen. Oftmals kommen die Patienten mit einer Vielzahl von Verletzungen und sind gerade nach Verkehrsunfällen polytraumatisiert.

Für einen komplizierten posttraumatischen Verlauf sind, bezogen auf die Sprunggelenke, vor allem höher gradig offene Frakturen und Gelenkfrakturen des Pilon-tibiale, des Kalkaneus und des Talus von Bedeutung. Gerade diese Verletzungen sind es, die in der Unfallchirurgie den Großteil der Patienten ausmachen, die später an einer posttraumatischen Arthrose leiden.

Die persistierende und therapierefraktäre posttraumatische Arthrose ist Hauptindikationsstellung zur Arthrodese.

Es ist trotz ausgereifter Osteosyntheseverfahren mit exakter Rekonstruktion der Gelenkflächen nicht immer möglich, eine posttraumatische Arthrose zu verhindern (Thermann et al. 1999).

Bei einer sekundären posttraumatischen Gelenkveränderung steht v.a. die subjektive Beschwerdesymptomatik des Patienten im Vordergrund. Dies geht mit einer entsprechenden Einschränkung im privaten und beruflichen Leben einher.

Der Leidensdruck für den Patienten ist hierbei oftmals sehr hoch und belastend.

Da es heute oft noch keine überzeugenden Behandlungsalternativen gibt, stellt die Arthrodese in den genannten Fällen die Therapie der Wahl dar. 
Das Ziel dieser Behandlung ist dabei eindeutig: größt mögliche Schmerzreduktion, zusammen mit dem Wiedererlangen von Stabilität und Sicherheit. Dabei sollte eine ausreichende Mobilität bei möglichst geringer Gehbehinderung erzielt werden (Rehart et al. 1999).

Dies ist ein subjektives Ziel, das jeder Patient individuell für sich definiert.

Der alternativ zur Verfügung stehende endoprothetische Gelenkersatz stellt meist keine gute Alternative dar (Kitaoka und Patzer 1996).

Für einen endoprothetischen Gelenkersatz sind eine gut erhaltene Funktion, Gelenkkontur- und Strukturerhalt Voraussetzung. Da dies selten gegeben ist, stehen nichttraumatische Indikationen für die Endoprothetik im Vordergrund (Horn 2003).

\section{Grundlagen}

\subsection{Topographische und funktionelle Anatomie der Sprunggelenke}

Das Sprunggelenk des Fußes besteht aus einem oberen (OSG, Abb.1/2) (Articulatio talocruralis) und einem unteren Sprunggelenk (USG). Das untere Sprunggelenk kann weiter in die Articulatio subtalaris und die Articulatio talocalcaneonavicularis unterteilt werden.

Die Gelenkfläche des oberen Sprunggelenkes wird durch die Malleolengabel auf der Trochlea tali gebildet. Die Malleolengabel wird durch den funktionellen Zusammenschluss von der Facies articularis inferior tibiae und dem Malleolus medialis der Tibia zusammen mit der Facies articularis malleoli lateralis der Fibula gebildet (Platzer 2003). Der Malleolus lateralis steht etwa $1 \mathrm{~cm}$ tiefer, als der Malleolus medialis der Tibia und bietet somit ein Gleitlager für die Peronealsehnen (Rammelt et al. 2004). An der lateralen Seite der distalen Tibia befindet sich die Incisura fibularis, wo die medial konvexe Seite der distalen 
Fibula liegt. Für den Zusammenhalt der beiden Unterschenkelknochen sorgen die jeweils anterior und posterior gelegenen tibiofibularen Bänder. Zusätzlich gibt es einen straffen Bindegewebszug direkt zwischen distaler Tibia und Fibula, der Ligamentum tibiofibulare interosseum genannt wird. Dieses ist für einen Zusammenhalt der Malleolengabel sehr wichtig und kann als eine nach distal gehende Verstärkung der Membrana interossea verstanden werden (Boldin et al. 2004; Rammelt et al. 2004). Verstärkend wirkt die Syndesmosis tibiofibularis und die Membrana interossea. Diese Bandstrukturen wirken auf axiale Belastungen, die die Malleolengabel auseinanderdrücken würden, ausgleichend und federnd (Müller-Gerbl 2001; Platzer 2003; Rammelt et al. 2004; Rammelt et al. 2008). Die Malleolengabel, als konkaver Gelenkanteil des Unterschenkels, stülpt sich über die allerdings hierzu inkongruente und konvexe Talusrolle (Seiler 1999; Koebke 2004). Die Talusrolle ist ventral ca. 4-5 mm breiter als dorsal und ist auf ihrer fibularen Seite konvex geformt. So entsteht an der lateralen Talusfläche eine Ellipse, wohingegen die mediale Gelenkfacette eine Kegelfläche besitzt. Somit lässt sich eine Pseudorotation bei Dorsalextension bzw. Plantarflexion des Gelenkes erklären (Rammelt et al. 2008). Weiter kann festgestellt werden, dass die tibiale Gelenkfläche etwas flacher als die Talusrolle ist (Müller-Gerbl 2001; Koebke 2004; Rammelt et al. 2008).

Starke mediale und laterale Bänder schützen das Gelenk vor Luxationsverletzungen. Medial befindet sich das am stärksten ausgeprägte Band, das sogenannte Ligamentum deltoideum, was auch Ligamentum collaterale mediale genannt wird. Dieses besteht aus vier Anteilen, der Pars tibionavicularis, einer Pars tibiocalcanea und einer Pars tibiotalaris anterior und posterior (McRae 1995; Seiler 1999; Platzer 2003). Dieses mediale Außenband beschränkt eine Pronationsbewegung im USG und verhindert ein Aufklappen des OSG (Müller-Gerbl 2001). Auf der lateralen Seite des OSG verlaufen drei jeweils getrennte Ligamenta, die als funktionelle Einheit verstanden werden. Das laterale Außenband, auch Ligamentum collaterale laterale genannt, gliedert sich in das Lig. talofibulare anterius und posterius und das Lig. calcaneofibulare. 
Funktionsaufnahmen haben gezeigt, dass bei Dorsalextension hauptsächlich das Lig. talofibulare posterius angespannt wird, während bei der Plantarflexion vor allem eine Spannung in dem Lig. calcaneofibulare stattfindet. Das sich in der Mitte von beiden befindliche Lig. talofibulare anterius wird bei beiden Bewegungen etwas, hauptsächlich jedoch bei Varusstress belastet.

Das Lig. deltoideum wird hauptsächlich bei Valgusstress belastet und schützt vor einem medialen Abknicken. Statische Belastungen stehen hier im Vordergrund. Die lateralen Bänder begrenzen die Rotation der Fibula und werden bei Valgusbelastung gedehnt (McRae 1995; Seiler 1999; Müller-Gerbl 2001).
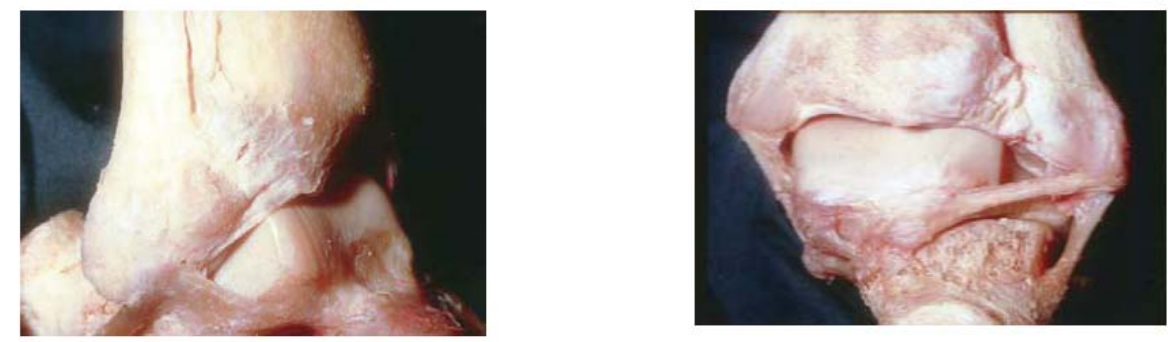

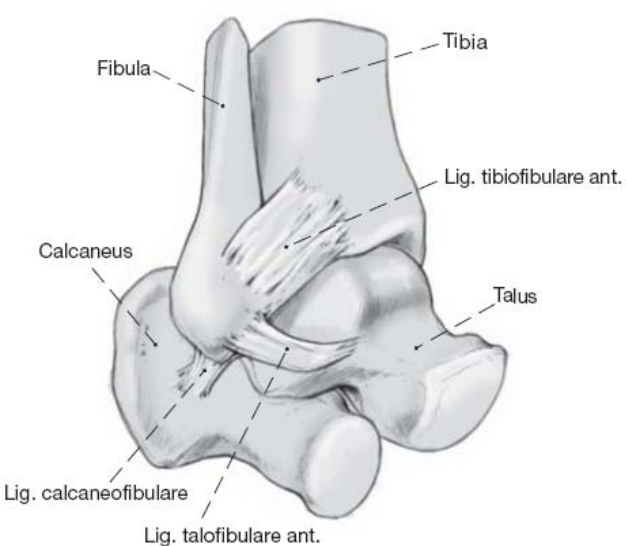

Abb.1 Ansicht der Anatomie des OSG von lateral

(Boldin et al. 2004, S.434)

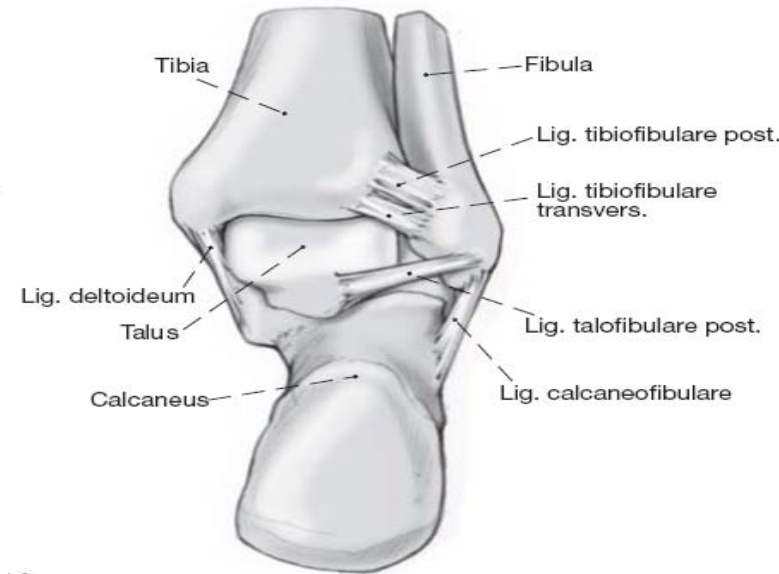

Abb.2 Ansicht der Anatomie des OSG von dorsal

(Boldin et al. 2004, S.434) 
Es ist möglich, mit dem oberen Sprunggelenk eine Dorsalextension bzw. eine Plantarflexion mit einem maximalen Bewegungsumfang von bis $\mathrm{zu} 70^{\circ}$ durchzuführen.

Das untere Sprunggelenk besteht, wie oben beschrieben, aus zwei funktionellen Einheiten. Zunächst bildet die Art. subtalaris den Anteil zwischen Talus und Kalkaneus. Die Art. talocalcaneonavicularis ist das zur Fußwurzel gerichtete Gelenk und verbindet drei Gelenkkörper miteinander und ist ein funktionelles Kugelgelenk (Pisani 1998).

Der Kalkaneus besteht aus mehreren Anteilen. Ein nach dorsal ausgerichteter starker Fortsatz, der Tuber calcanei, nimmt die Triceps- surae- Sehne auf. Am Processus medialis tuberis calcanei ist der Ansatz der Plantaraponeurose. Das Sustentaculum ist von seiner Trabekelstruktur her der anatomisch und biomechanisch stabilste Teil. Es dient dem Talus als medialer Abstützpfeiler. Mit dem Talus ist es fest durch die Ligg. talocalcanea mediale und calcaneotibiale verbunden. Bei einem Unfall bleibt der Talus meist mit dem Kalkaneus verbunden, wobei der Kalkaneus höchstens varisch abkippen kann.

Der Processus anterior calcanei bietet über die Ligg. calcaneocuboidale dorsale und bifurcatum eine feste ligamentäre Verbindung zum Os cuboideum und Os naviculare. Für die beiden Peronealsehnen bietet die Trochlea peronealis auf der lateralen Seite des Fersenbeins als kleinster Fortsatz zusammen mit dem Retinaculum peroneale diatale und dem Sulcus peronealis eine feste Führung (Zwipp und Grass 2005; Zwipp et al. 2005). Der Talus trifft im USG auf den Kalkaneus und das Os naviculare. Das Lig. calcaneonaviculare plantare bildet eine weitere, von Faserknorpel bedeckte Gelenkfläche. Dieses Pfannenband bildet zusammen mit dem Os naviculare eine Art Pfanne für die facies articularis des Kalkaneus (Platzer 2003).

Das durch den Sinus tarsi verlaufende Lig. talocalcaneum interosseum trennt das vordere von dem hinteren unteren Sprunggelenk. Ein kräftiges Lig. bifurcatum verstärkt die Kapsel und verbindet Kalkaneus, Os naviculare und das Os cuboideum (Pisani 1998; Platzer 2003). Zwischen dem Kalkaneus und dem ventral davon liegenden Os cuboideum verläuft die Chopart'sche Gelenklinie, die sich nach medial zwischen dem Talus und dem Os naviculare 
fortsetzt (Platzer 2003). Insgesamt ist das untere Sprunggelenk ein Zapfengelenk (Art. trochoidea), das vor allem die Pro- und Supinationsbewegung zulässt. Der Gesamtbetrag des Bewegungsumfanges beträgt in etwa $60^{\circ}$ zwischen den Extremstellungen (Platzer 2003).

Für einen sicheren Stand auf verschiedenen Untergründen ist eine eingeschränkte USG- Beweglichkeit erforderlich (Pisani 1998).

Die oben beschriebene Supinationsbewegung des Fußes kann als ein kombinierter Bewegungsablauf aus Innenrotation, Inversion, Plantarflexion und Adduktion verstanden werden.

Die Inversion an sich stellt eine Art Ausgleichsbewegung dar. Es beschreibt eine Überlagerung von Supination, Adduktion und Plantarflexion. Praktisch geschieht dies, wenn das Subtalargelenk in Varusstellung gelangt. Daraufhin folgt der Fuß mit einer Adduktion und Plantarflexion. Genauso kann die Pronation des Fußes als Außenrotation, Eversion, Dorsalextension und Abduktion verstanden werden (Debrunner und Hepp 1994; McRae 1995; Rehart et al. 1999; Rammelt et al. 2004).

Die Eversion setzt sich aus Dorsalextension und Abduktion zusammen. Das sieht praktisch so aus, dass, wenn im Subtalargelenk eine Valgisierung herbeigeführt wird, der Fuß mit einer Dorsalextension und Abduktion folgt (Debrunner und Hepp 1994; McRae 1995; Rammelt et al. 2004).

Die Wölbung des Fußes kann in eine mediale und eine laterale eingeteilt werden. Die laterale Seite ist hierbei eine konstant belastete Stützwölbung. Medialseitig besteht eine variabel belastete Bewegungswölbung, die bei Supination zunimmt, bzw. bei Pronation abnimmt.

Diese Wölbung wird unter inaktiven Bedingungen durch die Ligamenta, die Plantaraponeurose, sowie durch die Bindegewebssepten des Fußes einerseits und durch die langen und kurzen Fußmuskeln andererseits unterstützt (Pisani 1998).

Das untere und obere Sprunggelenk sind somit eine funktionelle Einheit (Koebke 2004).

Die Belastung wird so über das obere Sprunggelenk bzw. den Talus aufgenommen und daraufhin über das USG auf den restlichen Fuß verteilt 
(McRae 1995). Die Kräfte fokussieren sich dabei im OSG hauptsächlich auf die Facies superior der Trochlea tali und die distale Tibiagelenkfläche. Die Möglichkeit der Dorsalextension bzw. Plantarflexion ist für den biomechanischen Ablauf der Fußbewegung von eminent wichtiger Bedeutung. Ein Verlust dieser Funktion, beispielsweise durch eine Arthrodese, kann durch andere Gelenke nicht ausgeglichen werden (Koebke 2004).

\subsection{Sprunggelenksverletzungen}

Bei einem Unfall sind die Sprunggelenke bzw. der distale Unterschenkel häufig mit verletzt. Eine genaue Kenntnis der Anatomie, des Unfallhergangs und des Ausmaßes der Verletzung sind daher Voraussetzung für eine präzise Indikationsstellung. Einfache Frakturen des medialen oder lateralen Außenknöchels, sowie mediale oder laterale Bandverletzungen können unter bestimmten Voraussetzungen funktionell-konservativ behandelt werden. Dafür muss jedoch sichergestellt sein, dass es sich nicht um eine Luxationsverletzung handelt. Die anatomischen Strukturen müssen noch physiologisch präzise zueinander stehen, so dass die Malleolengabel keine Instabilität aufweist. Es darf keinesfalls im Bereich der Gelenke zu einer wenn auch nur minimalen Stufenbildung kommen. Diese würde das Risiko einer posttraumatischen Arthrose drastisch erhöhen (Paul et al. 1968). Eine Luxationsfraktur stellt eine unmittelbare Notfallindikation zum Reponieren unter Analgesie dar. Dadurch können schwere Weichteilschäden verhindert werden. Eine exakte operative Reposition ist entscheidend, für die Minimierung des postoperativen Risikos, eine posttraumatische Arthrose zu entwickeln. Die anatomisch präzise Wiederherstellung der Malleolengabel steht gerade bei distalen Syndesmosenverletzungen mit der Rekonstruktion der distalen Fibula in die Inzisur der Tibia im Vordergrund. Es muss jedoch klar gesagt werden, dass

eine ungenügende Wiederherstellung der Anatomie bzw. eine zu späte Versorgung oder das Vorliegen von multiplen Verletzungen das Risiko für 
schlechte Ergebnisse erhöhen (Rammelt et al. 2008). Für eine Einsicht der Klassifikationen mit Pathogenese der einzelnen Frakturtypen, siehe Anhang.

\subsection{Diagnostik}

\subsubsection{Klinische Untersuchung}

Bei Malleolarfrakturen zeigt sich bei der klinischen Untersuchung meist ein druckschmerzhaftes geschwollenes Gelenk. Wenn eine erhebliche Schwellung vorliegt, muss immer an ein Kompartmentsyndrom gedacht werden und u.U. eine Druckmessung durchgeführt werden. Die Untersuchung von peripherer Durchblutung, Motorik und Sensibilität sollte dabei immer durchgeführt werden. Die Beweglichkeit ist in jedem Fall eingeschränkt, oft sogar durch Dislokation und Schmerz etc. unmöglich. Axiale Fehlstellungen und prominente Knochenfragmente sind bei der Luxationsfraktur nahezu obligat. Um einen Maisonneuve-Fraktur-Verdacht auszuschließen, sollte die Fibula in ihrer ganzen Länge palpiert werden. Ist keine Druckschmerzhaftigkeit vorhanden, so ist dies jedoch kein Ausschlusskriterium. Ergänzend sollten die Innen- und Außenknöchel palpiert werden. Dabei deuten Schmerzen oder Fehlstellungen auf Frakturen hin (Zwipp et al. 2005; Rammelt et al. 2008).

\subsubsection{Bildgebung}

Die konventionelle Röntgenuntersuchung des OSG erfolgt mindestens in 2 Ebenen. Hierbei erfolgt eine Aufnahme exakt von der Seite. Die andere Aufnahme wird mit einem anteroposterioren Strahlengang bei einem um $20^{\circ}$ nach innen gedrehten Unterschenkel angefertigt. Dadurch kann der Gelenkspalt und die Knöchelgabel frei dargestellt werden. Hierbei ist auf einen gleichmäßig weiten medialen und lateralen Gelenkspalt und auf den Abstand zwischen Fibula und Tibia $1 \mathrm{~cm}$ proximal des Gelenkspaltes zu achten. Dieser tibiofibulare Abstand wird „ligne claire“ ( oder espace claire) nach Chaput oder 
„tibiofibular clear space“ genannt (Abb.3). Des Weiteren ist auf die tibiofibulare Überlappung zu achten. Als Hinweis auf eine Syndesmosenverletzung oder eine chronische Insuffizienz gilt eine „ligne claire“ größer als $5 \mathrm{~mm}$. Wenn eine chronische Syndesmoseninsuffizienz besteht, wird häufig zusätzlich eine arthrotische Veränderung und/ oder Ossifikation auf Höhe der Syndesmose gesehen. Bei fraglicher Syndesmoseninsuffizienz sollte eine Aufnahme der Gegenseite gemacht werden. Bei einer Differenz von min. $2 \mathrm{~mm}$ wird dies als Syndesmoseninstabilität aufgefasst (Xenos et al. 1995; Grass et al. 2000). Das Maß der tibiofibularen Überlappung kann als Korrelat zur Stabilität der Syndesmose betrachtet werden. Dieser "Overlap" sollte bei $20^{\circ}$ Innenrotation min. $1 \mathrm{~mm}$, bei a.-p. Strahlengang min. $6 \mathrm{~mm}$ oder 40\% der Tibiabreite sein. Der Verdacht auf eine Subluxation des Talus kann durch einen erweiterten "medial clear space" (Erweiterung des medialen Gelenkspaltes) im Vergleich zum oberen Gelenkspalt entstehen. Diese mediale Gelenkspaltweite sollte im Normalfall kleiner als $4 \mathrm{~mm}$ sein (Xenos et al. 1995).

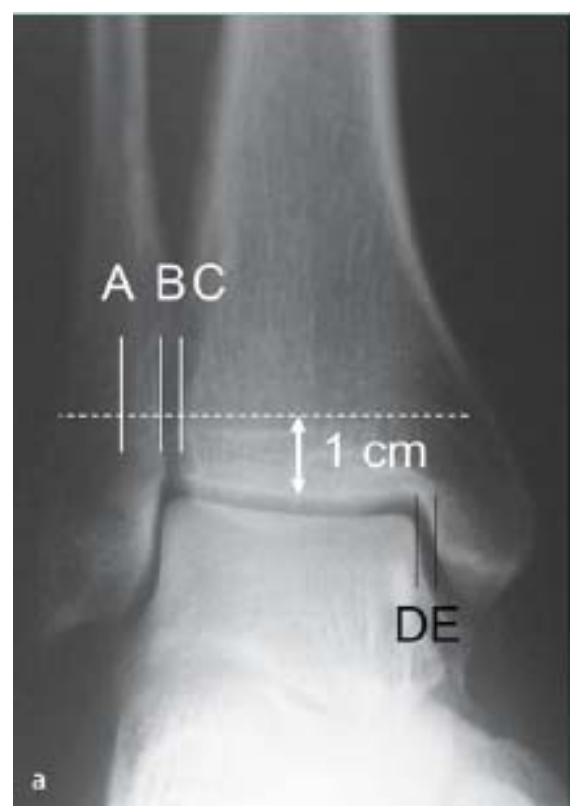

Abb.3 OSG im a.-p. Strahlengang mit $20^{\circ}$ Innenrotation (Rammelt et al. 2008 S.427)

A- tibiofibulare Überlappung

B-C: „ligne claire“ tibiofibulare Distanz

D-E: Gelenkspaltweite medial 
Die Syndesmose kann dynamisch unter dem Bildwandler mit dem Frick-Test untersucht werden. Hierbei fasst eine Hand des Untersuchers den Unterschenkel proximal des USG von lateral an. Mit der anderen Hand wird der Fuß von medial umfasst und nach lateral gedrückt. Wenn die Syndesmose insuffizient ist, kommt es zu einer deutliche Aufweitung des medialen Gelenkspaltes (Grass et al. 2000; Rammelt et al. 2003). Wenn weitere Strukturen wie Talus oder USG verletzt sind, oder der Verdacht auf eine Pilontibiale Fraktur besteht bzw. schwer zu klassifizierende Frakturen, sollte zur Sicherheit ein CT durchgeführt werden. Zur OP-Planung von Syndesmosenstabilisierungen sollte ein $\mathrm{CT}$ in sagittaler Ebene durchgeführt werden (Rammelt et al. 2003). Gerade bei der Klärung der Frage nach einer instabilen oder verletzten Syndesmose ist die CT- Untersuchung die sensitivste Methode (Xenos et al. 1995). Um eine Subluxationsstellung des Talus nach lateral am besten objektivieren $\mathrm{zu}$ können, hat sich eine vergleichende Beurteilung des Syndesmosenspaltes in einer frontalen Rekonstruktion (im CT) bewährt. Dies ermöglicht dann eine gute Indikationsstellung für eine operative Korrektur (Grass et al. 2000). Das Interesse der Kernspintomographie an der Darstellung von Syndesmosenverletzungen hat in letzter Zeit zugenommen. Dieses Verfahren kann suffizient die ligamentären Strukturen des distalen Unterschenkels darstellen (Muhle et al. 1998). Da das MRT im Vergleich zum CT keine therapierelevanten Zusatzinformationen liefert, ist es für die Routinediagnostik nicht zu empfehlen (Zwipp et al. 2005). Um USG-Frakturen auszuschließen, wird in 3 Ebenen geröngt: Kalkaneus lateral, axial und Fuß dorsoplantar (Zwipp et al. 2005). Eine suffiziente Beurteilung des Frakturverlaufs bei USG- Verletzungen kann jedoch nur durch das CT erfolgen. Denn nur im CT ist eine genaue Analyse der Frakturmorphologie und damit eine präzise OP- Planung möglich (Zwipp et al. 2005; Reiser et al. 2006). 


\subsection{Indikationsstellung}

\subsubsection{Konservative Therapie}

Bei stabilen nicht dislozierten Innen- und Außenknöchelfrakturen ist eine konservative Therapie möglich. Hierzu zählen unter anderem folgende:

- Nach AO Typ-A-1, (Supinations-Adduktionstraumata): einfache infrasyndesmale Fibulafrakturen

- Nach AO Typ-B-1, (Supinations-Eversionstraumata): stabile transsyndesmale Fibulafrakturen ohne eine Syndesmoseninstabilität

- Pronations-Eversionstraumata: stabile, nicht dislozierte Innenknöchelfrakturen

(Rammelt et al. 2007).

Bei extraartikulären, nicht dislozierten Frakturen des Fersenbeins kann primär konservativ vorgegangen werden. Es gibt jedoch zwei als instabil anzusehende Frakturen, die sich nach Zwipp et al. (2005) sekundär nahezu regelhaft dislozieren und daher operativ angegangen werden sollten. Dieses sind die 2Teile-Scherfrakturen und die isolierten Sustentakulumfrakturen (Wenzl et al. 2001; Zwipp et al. 2005). Eine Konservative Behandlung wird empfohlen bei:

- stark kompromittierten Weichteilen (Kontusionen, Blasenbildungen, Infekte)

- insulinpflichtigem Diabetes mellitus

- peripherer arterieller Verschlusskrankheit

- Polyneuropathie

- Alkohol- und Drogensucht

- Immunsuppressiver Behandlung

- exzessivem Nikotinabusus (nach Sanders 2000)

(Zwipp et al. 2005). 
Vor einer konservativen Therapie müssen Schäden, die eine Instabilität hervorrufen, ausgeschlossen werden. Hierzu gehören ligamentäre Schäden wie die Syndesmosen-Verletzungen und ein knöcherner Ausriss des Tuberculum tibiae posterior. Ein besonderes Augenmerk sollte auf das mediale Kompartiment gerichtet werden, um sicher zu sein, dass das Deltaband oder der mediale Knöchel unverletzt sind. Bewährt hat sich im Zweifelsfall der manuelle Stresstest unter dem Bildwandler. Die klinische Erscheinung von Strukturverletzungen der medialen Seite wie Schwellung, Ekchymosen oder Schmerz liefern keine sichere Aussage zur Stabilitätsfrage (Egol et al. 2004; McConnell et al. 2004). Wenn unter dem Bildwandler die Verschieblichkeit auf der lateralen Seite mehr als $2 \mathrm{~mm}$ beträgt, so stellt dies ein Instabilitätskriterium dar (Richter et al. 1999). Es ist möglich, die Stabilitätsprüfung unter leichtem Außenrotationsstress bei fixiertem Unterschenkel durchzuführen. Auf eine instabile Knöchelgabel lässt sich schließen, wenn der mediale Gelenkspalt breiter als $4 \mathrm{~mm}$ wird, oder aber der mediale Gelenkspalt im Gegensatz zur Gegenseite um mehr als $1 \mathrm{~mm}$ breiter wird (Phillips et al. 1985; McConnell et al. 2004).

\subsubsection{Operative Therapie}

Für alle instabilen und dislozierten Verletzungen ist die operative Reposition und Osteosynthese die Therapie der Wahl. Ziel ist es, durch eine präzise Wiederherstellung der Gelenkkongruenz und der Stabilität das Risiko für eine potentielle posttraumatische Arthrose zu minimieren (Rammelt et al. 2007).

Heute ist ein Lateralversatz, eine Verkürzung der Fibula um mehr als $2 \mathrm{~mm}$ und eine Rotationsfehlstellung von mehr als $5^{\circ} \mathrm{im}$ Vergleich zur gesunden Gegenseite als Operationsindikation anzusehen. Ohne Operation würde das Risiko einer Arthrose zu stark steigen (Thordarson et al. 1997).

Eine operative Versorgung sollte ebenfalls angestrebt werden, wenn bei Kalkaneusfrakturen intraartikuläre Beteiligungen mit Gelenkstufen von mehr als $1 \mathrm{~mm}$ an der posterioren, medialen oder kuboidalen Facette oder extraartikuläre 
Frakturen mit Rückfußfehlstellungen varisch $>5^{\circ}$ oder valgisch $>10^{\circ}$ auftreten (Zwipp et al. 2005).

\subsection{Historie der Arthrodese}

Der Begriff Arthrodese kommt aus dem Griechischen und steht für eine feste Gelenkbindung (Jerosch et al. 2006)

Die erste Arthrodese wurde von dem Innsbrucker Chirurgen Eduard Albert (1879) beschrieben, der bei einem 17 jährigen Jungen mit postinfektiöser, spitzwinkliger Kniekontraktur eine Kniegelenksresektion mit vollständiger Naht und primärer Vereinigung durchführte. Damals verband Albert (1879) Tibia und Femur mit einer Silberdrahtnaht. Der Begriff der Arthrodese wurde erst später durch einen seiner Mitarbeiter geprägt. Dies war der Anstoß für weitere operative Gelenkversteifungen. Das erste Sprunggelenk (OSG) versteifte er 1879 bei einem 14 jährigen Mädchen mit poliomyelitischer Lähmung mit Fußdeformität, was früher die vornehmliche Indikation für eine operative Gelenkversteifung darstellte. Es sind vielfältige Techniken und Varianten der Arthrodese entwickelt worden, beispielsweise werden über 50 Arthrodesevarianten beschrieben. Die Varianten der Arthrodese können in externe und interne osteosynthetische Verfahren unterteilt werden (Baciu und Filibiu 1979; Scranton 1985; Russotti et al. 1988; Moeckel et al. 1991; Myerson und Quill 1991; Scranton 1991; Jerosch et al. 1996; Bhan und Malhotra 1998; Rowan und Davey 1999; Thermann et al. 1999; Jerosch und Vollmert 2005). Es konnte biomechanisch in Bezug auf Torsions- und Biegesteifigkeit gezeigt werden, dass die internen gegenüber den externen Operationsverfahren im Vorteil sind (Moeckel et al. 1991; Thordarson et al. 1992; Zwipp et al. 1999; Buchner und Sabo 2003 b).

Erst ab ca. 1951 wurden Fortschritte bei dem Problem der Fixation der Resektionsflächen gemacht.

Zunächst wurde hier die Kompressionsarthrodese mittels Spanbügel eingeführt. 
Charnley (1951) hat die Kompressionsarthrodese mittels Fixateur Extern fortentwickelt. In der Praxis wies dieses Verfahren noch viele Schwächen auf, v.a. eine hohe Rate an Infektionen. Charnley (1951) konnte jedoch das erste Mal eine gewisse Kompression der Knochenflächen mittels Einbringen von zwei Drähten proximal und distal des Arthrodesespaltes erzeugen. Gute Erfolge mit diesem Kompressionsverfahren ermutigten Charnley (1951), dieses am Sprunggelenk zu versuchen. Die Tatsache, dass die Patienten schneller wieder mobilisiert werden konnten, bestärkte Charnley (1951) in seiner Vorstellung, eine gute Technik entwickelt zu haben. Als Weiterentwicklung durch Zuhilfenahme von interner Fixation führte Allgöwer die Spongiosazugschrauben ein (Jerosch und Vollmert 2005). Eine Weiterentwicklung fand Anfang der 1970er Jahre statt. Hier wurden erstmalig offiziell Kompressionswinkelplatten von Mittelmeier et al. (1975) eingesetzt.

Zur gleichen Zeit begründete Zimmermann die Zugschraubenosteosynthese (Stuhler 1994).

In einer Weiterentwicklung konnte diese OP-Methode für die arthroskopisch assistierte Arthrodese (AAA) gut verwendet werden (Coester et al. 2001; Jerosch und Vollmert 2005). Die AAA ist speziellen Indikationen vorbehalten. So darf keine manifeste Fehlstellung oder ein Substanzdefekt der Gelenkpartner vorliegen. Des Weiteren sollten Patienten mit neurogenen Arthropathien nicht mit dieser Methode operiert werden. So wird dieses Verfahren als eine „in-situ“-Fixation der zerstörten Gelenkpartner angesehen (Jerosch 2003).

In den 90er Jahren hat sich die tibiotalare Arthrodese durch intramedulläre Kompressionsnagelung etabliert. Die Vorteile liegen in einem geringen Weichteilschaden auf Sprunggelenkhöhe, der frühen axialen Belastbarkeit und einer Nachbehandlung ohne Gipsruhigstellung (Muckley et al. 2003).

Auf die neue Knochen-Dübel-Arthrodese (KDA) in press-fit-Technik wird auf Seite 40 und folgenden detailliert eingegangen. Der Arthrodese kommt somit wegen noch fehlender besserer Therapien eine wichtige Rolle in der Behandlung von sekundär posttraumatischen Gelenkveränderungen mit therapierefraktärer Schmerzsymptomatik zu. 


\subsection{Indikation zur Arthrodese}

Es ist heute trotz sehr ausgereifter Osteosyntheseverfahren nicht immer möglich, eine posttraumatisch symptomatische Arthrose zu vermeiden.

Der Patient steht dabei meist unter einem erheblichen Leidensdruck, der inn beruflich wie privat im ausgeprägten Maße einschränkt. Dabei stehen die meist therapierefraktären Schmerzen mit an oberer Stelle.

Die operative Gelenkversteifung spielt dabei aufgrund von fehlenden Alternativen eine wichtige Rolle (Müller E et al. 1999; Rehart et al. 1999; Thermann et al. 1999; Horn 2003).

Das Ziel der Arthrodese ist primär die Schmerzreduktion, die Wiederherstellung der Stabilität und die Sicherung einer ausreichenden Mobilität bei möglichst geringer Gehbehinderung (Mann et al. 1991; Chen et al. 1996; Müller et al. 1999; Rehart et al. 1999; Bonnaire et al. 2001).

Bei der Indikation sind Faktoren wie Alter, Gewicht, Beruf und Betätigungen im Privatleben $\mathrm{zu}$ beachten. Unabdingbar wichtig sind Faktoren wie die Beschwerdesymptomatik mit den evtl. täglichen Schmerzen. Darüber hinaus muss die Notwendigkeit Hilfsmittel in Anspruch nehmen zu müssen mit beachtet werden. Für den Patienten ist die Beeinträchtigung im Berufs- bzw. im Privatleben sehr relevant (Rehart et al. 1999; Jerosch und Vollmert 2005). Manche Autoren stellen die Indikation zur Arthrodese erst nach fehlgeschlagener konservativer (intraartikuläre Kortikoid-Injektion oder systemische NSAR-Therapie) bzw. chirurgischer Therapie mit Analgesie etc. (Scranton 1991). Die Arthrodese ist somit die chirurgische ultima ratio.

Heute wird dies meist notwendig bei symptomatisch posttraumatischer Arthrose, (nach Pilontrümmerfraktur/ Talusluxationsfraktur etc.) symptomatischen Arthrosen anderer Genese, Frakturen mit erheblicher Gelenkzerstörung, posttraumatischen Durchblutungsstörungen, chronisch posttraumatischer Gelenkentzündung (posttraumatischer Osteitis), 
rheumatischer Arthritis, Fehlstellung und Deformitäten infolge paralytischer bzw. spastischer Lähmungen und bei fehlgeschlagener Osteosynthese. Siehe folgende Abb.4 (Horn 2003):

- symptomatische posttraumatische Arthrosen

- symptomatische Arthrosen sonstiger Genese

- Frakturen mit erheblicher Gelenkflächenzerstörung

- posttraumatische Durchblutungsstörungen

- chronische posttraumatische Gelenkinfektionen

- rheumatische Arthritis

- Fehlstellungen und Deformitäten infolge paralytischer oder spastischer Lähmung.

Abb.4 vorrangige Indikationen zur operativen Sprunggelenks-Arthrodese (Horn 2003 S.179)

Vor der Durchführung einer Arthrodese ist die Infiltration des entsprechenden Gelenks mit Lokalanästhetika obligat. Eine vorübergehende Beschwerdefreiheit bzw. Minderung ist für den operativen Eingriff vorauszusetzen (Dresing und Stürmer 2000).

\subsection{Arthrodeseformen}

Zahlreiche Techniken zur Arthrodese werden beschrieben. Zu unterscheiden sind primäre und sekundäre Arthrodesen. Die primäre Arthrodese wird bei frischem Trauma mit nicht rekonstruierbaren Gelenkflächen durchgeführt. Dies gilt als sehr anspruchsvoll mit relativ schlechten Ergebnissen. Dies korreliert mit dem hier meist sehr dramatischen Krankheitsbild. Die frühe Arthrodese wird innerhalb eines halben Jahres nach dem Unfall durchgeführt. Die Spätarthrodese folgt erst viel später bei bereits manifester Arthrose. 
Eine Einteilung der Arthrodesen am Sprunggelenk wird nach der Anzahl der versteiften Gelenke vorgenommen. Die einfache oder monoartikuläre Arthrodese bezieht ein Gelenk ein, entweder das OSG oder das USG. Bei der double Arthrodese werden das OSG und USG kombiniert versteift. Darüber hinaus existiert die triple-Arthrodese, bei der folgende Gelenke versteift werden: subtalar-, talonavikular-, kalkaneokuboidal (Nieny 1905; Stuhler 1994; Horn 2003; Jerosch und Vollmert 2005). Wenn der Talus aufgrund des Traumas oder einer Durchblutungsstörung mit anschließender Nekrose entfernt werden muss, kommt es zu einer tibio- navikulo- kalkaneären Arthrodese (Horn 2003). Aus der Fülle heute einzeln existierender Arthrodeseverfahren wird ersichtlich, dass es eine optimale Methode nicht gibt (Morrey und Wiedeman 1980; Morgan et al. 1985; Moeckel et al. 1991).

\subsubsection{Die OSG-Arthrodese}

Technisches Ziel der OSG-Arthrodese ist die dauerhaft stabile Fixation des Gelenks. Dies wird erzielt durch eine Kompressionsverschraubung, die eine achsengerechte Einstellung des Fußes bzw. die Korrektur bestehender Achsenfehler erlaubt. Dafür ist eine möglichst gute Präparation vitaler spongiöser Oberflächen notwendig, damit eine möglichst gute Kompression der Arthrodeseoberflächen stattfinden kann (Müller et al. 1999; Horn 2003). Erstrebenswert ist ein möglichst anatomischer Gelenkkonturerhalt. Dafür werden die Gelenkflächen von Tibia und Talus entknorpelt und sparsam reseziert. Unter Kompression erfolgt eine gegeneinander gerichtete Fixierung und damit Stabilisierung. Bei schlechter Knochenqualität, ausgeprägter Gelenkinkongruenz und ausgeprägten Knochendefekten erfolgt eine Kombination mit einer Spongiosaplastik (Müller et al. 1999). Es gibt diverse Varianten der Präparation der Gelenkflächen. Am häufigsten wird der Knorpel reseziert und die knöchernen Strukturen belassen (Morgan et al. 1985; Holt et al. 1991). Des Weiteren ist es möglich die beiden Flächen plan-parallel abzutragen (Buck et al. 1987; Thordarson 1993). Darüber hinaus können die 
korrespondierenden Flächen in der Art einer "Chevron-Osteotomie“ V-förmig zueinander ausgerichtet werden (Marcus et al. 1983).

Aufgrund der höheren Infektionsrate entlang der Steinmann-Nägel (Pin-Infekt, schlechtere Fusionsrate) wird eine externe Fixation nur in Ausnahmefällen (z.B. beim Gelenkinfekt) verwandt (Grass und Zwipp 1998; Müller E et al. 1999; Buchner und Sabo 2003 b; Jerosch und Vollmert 2005).

Für das spätere Ergebnis ist die Stellung des Gelenks sehr wichtig (Buck et al. 1987). Als ideal wird für das OSG eine neutrale Plantar- bzw. Dorsalextionseinstellung für das OSG angesehen. Der Fuß sollte in etwa $5^{\circ}$ valgisiert und $5-10^{\circ}$ außenrotiert sein. Die Valgisierung sollte an die kontralaterale Seite angepasst werden (Morgan et al. 1985; Buck et al. 1987; Müller et al. 1999; Holz 2006). Darüber hinaus ist es von Vorteil, wenn der Fuß in Relation zur distalen Tibia nach dorsal versetzt ist. Daraus resultiert ein verkürzter Hebelarm des Fußes auf die Arthrodese und damit ein mechanisch besseres Ergebnis (Buck et al. 1987). Heute wird meist von dem Dorsalverschub des Talus Abstand genommen. Es wird sich daraus eine verbessert erhaltene Ganggeometrie versprochen, wo eine Beinverkürzung umgangen werden kann (Holz 2006; Jerosch et al. 2006). Zur Stabilisierung und Fixierung dient oft die Osteosynthese mit Kompressionsplatten (ab 1970 durch Mittelmeier) (1975) oder nach dem Prinzip der Zugschraubenosteosynthese (durch Zimmermann erstmals beschrieben) (Stuhler 1994). Des Weiteren gibt es die Möglichkeit der Verriegelungsarthrodese nach Campbell und Wilson. Sie beschreiben die Überwindung des Gelenkspaltes durch Verwendung eines Verriegelungsspans wie z.B. Knochen. Am häufigsten wird hier das Fibulatransplantat verwendet. Da die Verriegelungstechnik höhere Komplikationsraten aufweist, fällt die Wahl der Technik meist zu Gunsten der Kompressionsarthrodese, die 1951 von Charnley (1951) begründet wurde, aus (Jerosch und Vollmert 2005). Indikationen für die Verriegelungsarthrodese sind jedoch ein ausgeprägter knöcherner Defekt oder eine zu erwartenden Talusnekrose (Müller E et al. 1999). Bei der Kompressionsarthrodese gibt es verschiedene Variationen. Die Schraubenkompressionsarthrodese gilt dabei als Goldstandard (Zwipp 1994; 
Grass und Zwipp 1998). Darüber hinaus gibt es die Vier- Schrauben- Technik nach Zwipp und Grass (1998). Dabei werden über einen ventromedianen Zugang zwei Schrauben von tibial nach talar eingebracht. Eine dritte Schraube verläuft vom posteromedialen Maleolus medialis zum anterolateralen Taluskopf. Die vierte Schraube wird vom Außenknöchel zum Taluskörper gedreht (Abb.5).

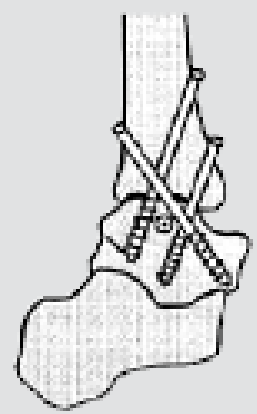

OSG

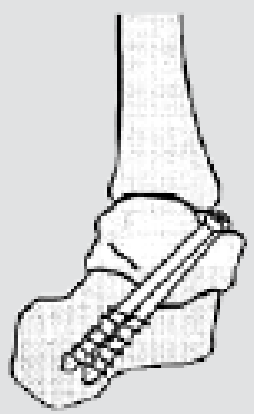

USG

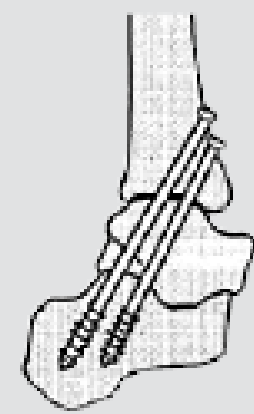

Double

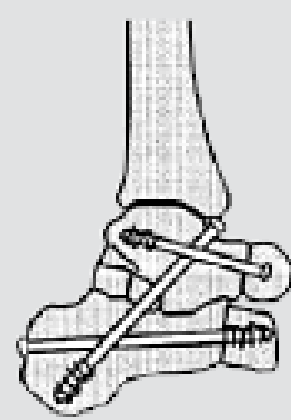

Triple

Abb. $1 \mathbf{\Delta}$ Arthrodeseformen im Sprunggelenkbereich [18]

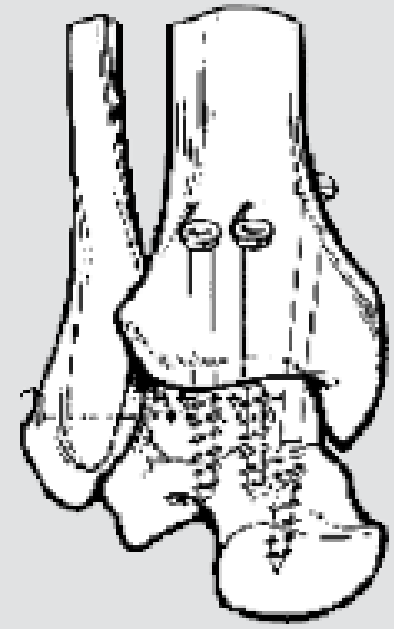

a

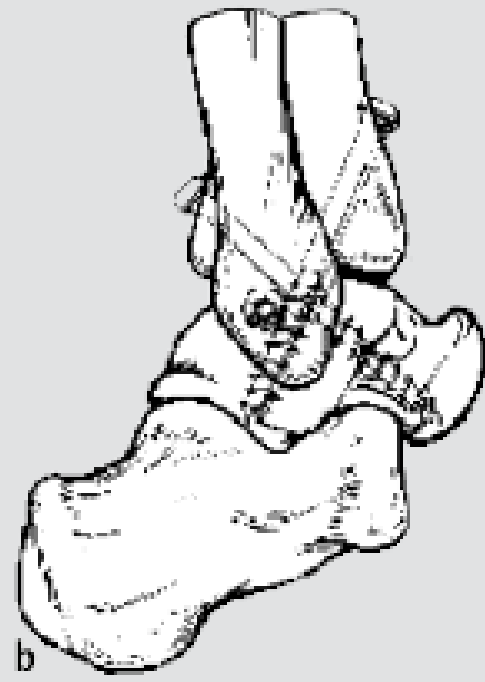

Abb. $2 \mathrm{a}, \mathrm{b}, 4$

4-Schrauben-Technik nach Zwipp [18]

Abb.5 Abb.1: Arthrodeseformen im Bereich der Sprunggelenke

Abb.2: 4- Schrauben -Technik nach Zwipp (Horn 2003 S.180)

Eine weitere Kompressionsarthrodese stellt die von Müller E (1999) beschriebene "Zwei-gekreuzte-Schrauben-Technik" mit einer optional zusätzlichen ventralen Platte dar. Hierbei wird eine Schraube auf der posterolateralen Tibiaseite von kranial nach anteromedial in den Talushals 
geschraubt. Von anteromedial nach posterolateral in die Talusrolle verläuft die zweite Schraube. Bei zweifelhafter Stabilität wird dies dann noch durch eine anteriore Platte ergänzt.

Die Kompressionsarthrodese mittels Fixateur extern sollte nur in Ausnahmefällen in Erwägung gezogen werden. Eine weitere Indikation für die externe Fixation ist eine zerstörte oder instabile lokale Anatomie. In diesem Fall würde das Einbringen von Schrauben bzw. Platten keine ausreichende Stabilität und Kompression erbringen (Zwipp 1994; Grass und Zwipp 1998; Jerosch und Vollmert 2005). Die Arthrodese des OSG mit anterogradem oder retrogradem Kompressionsmarknagel ist eine Methode mit einem lokal relativ geringen Zugangstrauma. Zusätzlich wird für dieses Verfahren ein kleiner Zugang an der proximal-ventralen Tibia kurz unterhalb der Tuberositas tibiae benötigt. Die korrespondierenden Gelenkflächen werden vorher entknorpelt und bis zu einem vital spongiösen Knochen angefrischt. Der Marknagel wird so meist von der proximalen Tibia durch den Markraum, bei OSG-Arthrodese bis in den Talus und bei USG-Arthrodese bis in den Kalkaneus, eingebracht. Daraufhin wird der Marknagel distal durch zwei zueinander rechtwinklig stehende Schrauben von dorso-medial sowie von antero-lateral durch den Talus bzw. den Kalkaneus verriegelt. Dadurch, dass die Schrauben rechtwinklig zueinander verlaufen, ergibt sich eine Rotationsstabilität gegenüber der Tibia. Der Nagel wird nach proximal geschlagen und dort verriegelt. Der Arthrodesespalt wird über eine Kompressionsschraube komprimiert. Es bleibt festzustellen, dass der Patient bei dieser Methode frühfunktionell schon ab der zweiten postoperativen Woche axial belastet werden kann und dadurch angrenzende Gelenke nicht lange inaktiviert werden müssen (Muckley et al. 2003).

Als neuere Variante gilt die von Dresing und Stürmer (2000) in Göttingen entwickelte Knochen-Dübel-Arthrodese (KDA) in press-fit Technik (Abb.8). Hierzu werden aus dem Beckenkamm kortikospongiöse Knochendübel mit einem Durchmesser von etwa 10-12 mm mit Hilfe einer innen gekühlten Diamantfräse entnommen (Abb.6). Anschließend werden in die Gelenkflächen mit der Diamanthohlfräse Zylinder gefräst (Abb.7), in die die Knochendübel mit 
der press-fit Technik ein gebolzt werden. Dann werden zwei Zugschrauben von medial und lateral durch den Innen- und Außenknöchel transossär und transartikulär in den Proc. anterior tali und Proc. posterior tali eingebracht.

Wenn der Patient kooperativ ist bzw. wenn es sich um eine fortgeschrittene Einsteifung handelt, kann sogar auf die Zugschraubenosteosynthese verzichtet werden. Eine äußere Fixation ist hier meist nicht notwendig. Vorteile der KDA sind die rasche Konsolidierung, die Schonung der Weichteile und die Erhaltung der Gelenks- und Fußkontur sowie der Beinlänge und Beckenkontur. Allerdings sollte diese Methode nur bei aseptischen Eingriffen gewählt werden. Dieses Verfahren ist bei primären Arthrodesen nach einem frischen Trauma und bei deutlichen Fehlstellungen nicht anzuraten (Dresing und Stürmer 2000).

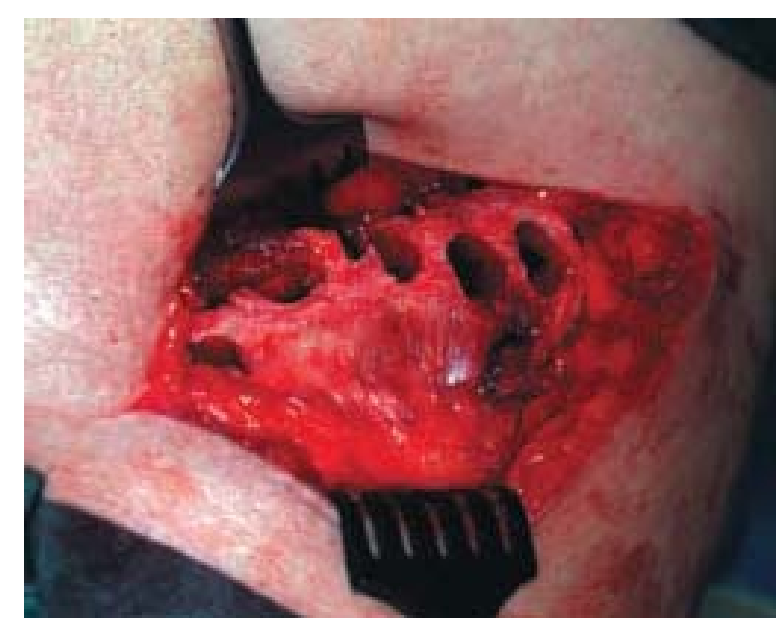

Abb.6 intraoperativer Blick auf den linken Beckenkamm nach Entnahme der Knochendübel (Dresing und Stürmer 2000 S.646) 

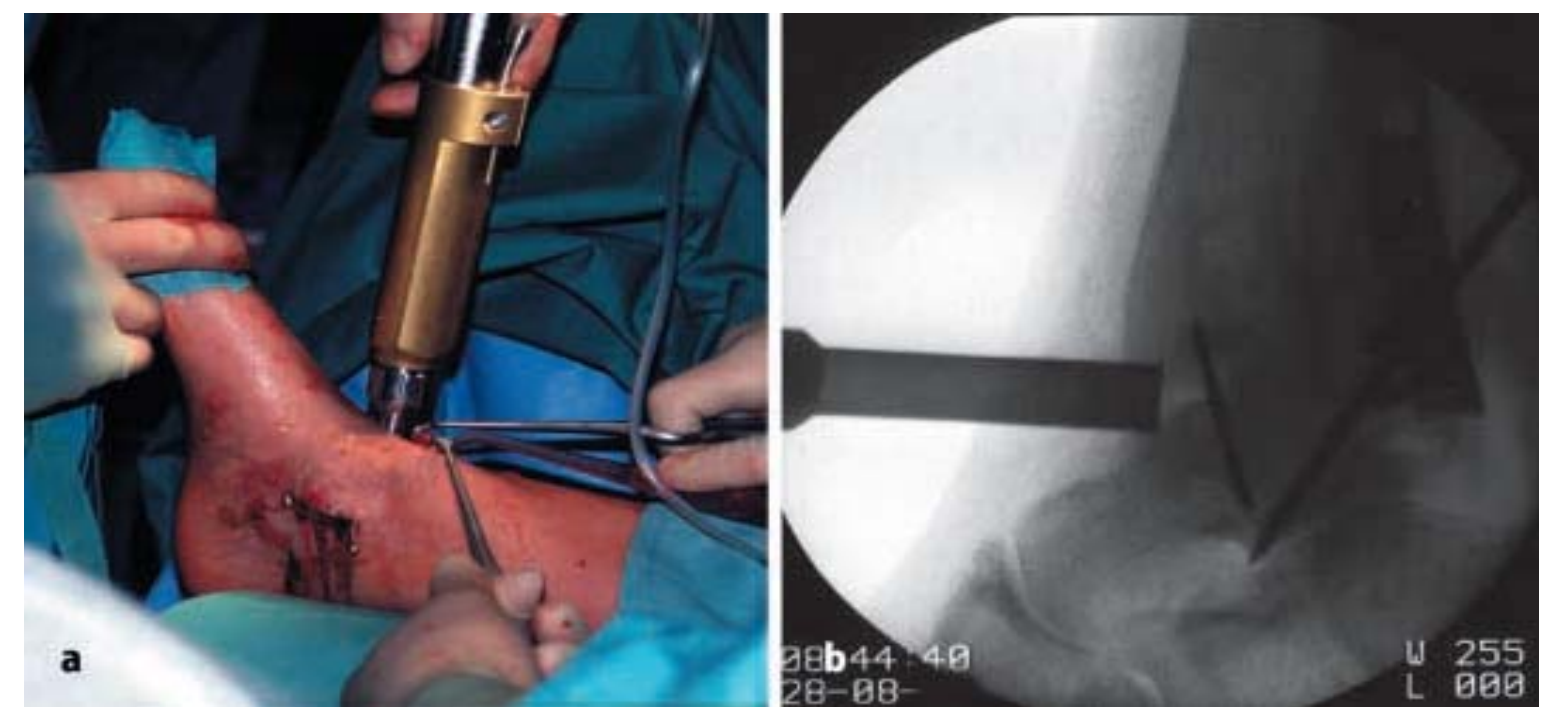

Abb.7: Abb. a: intraoperatives Bild: Fräsen des Bettes für einen ventralen Dübel. Die Diamantfräse ist an die AO- Bohrmaschine gekoppelt. Durch Bohrdrähte wird die OSG-Stellung retiniert. Abb. b: intraoperatives Röntgenbild (Bildwandler).

(Dresing und Stürmer 2000 S.647)

Das Verlaufsröntgenbild kann bei der KDA dem Ungeübten eine fälschlicherweise fehlende Integration vortäuschen, da die Kanäle am Empfängerort nicht immer komplett orthograd abgebildet werden. Es wird daher empfohlen in mehreren Projektionen zu röntgen; insbesondere ist für das USG zur Verlaufskontrolle die Brodén- Projektionsebene zu empfehlen (Dresing und Stürmer 2000). 


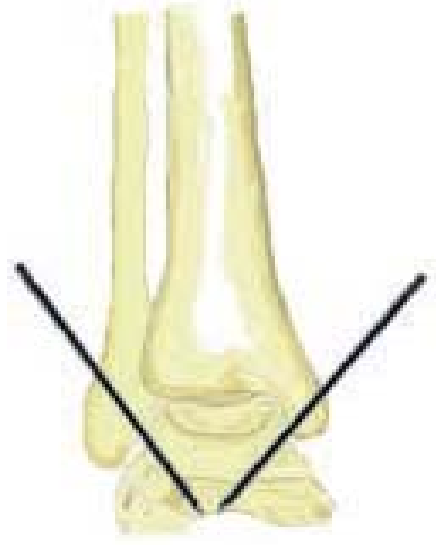

a

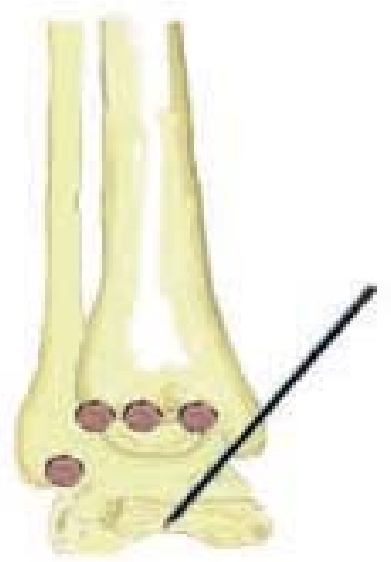

d

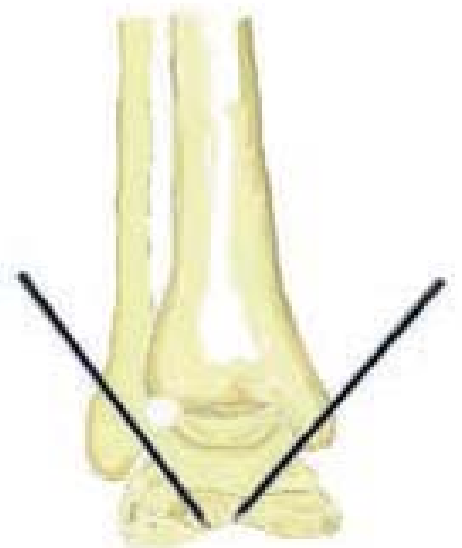

b

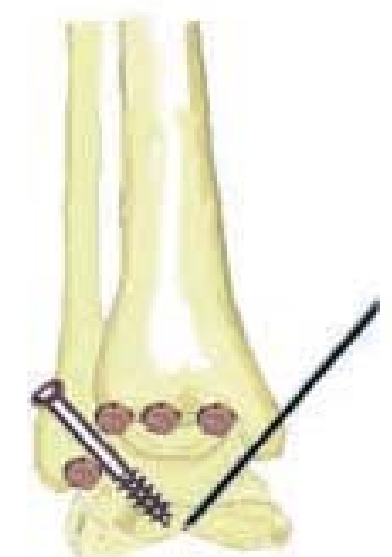

e

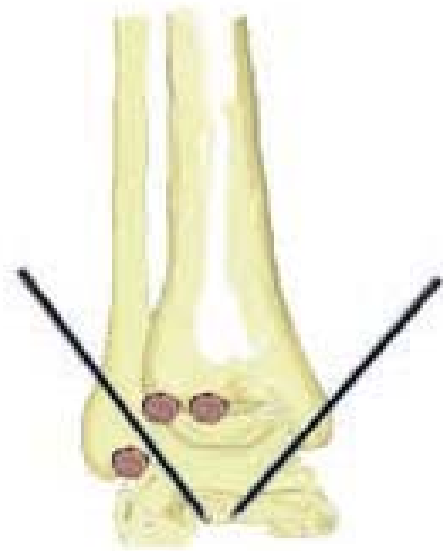

C

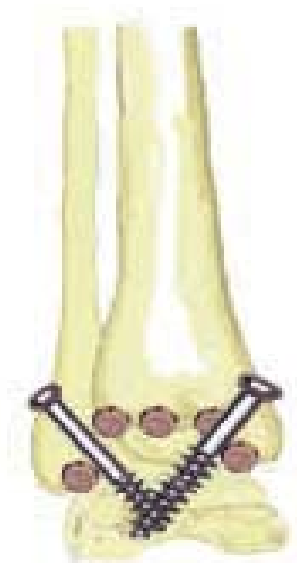

f

Abb.8: operatives Vorgehen bei der KDA-Arthrodese: a: durch 2 Bohrdrähte wird die Gelenkstellung retiniert. b: Fräsen eines zylindrischen Defektes von ventral. c: die Stärke der Fräse ist so zu wählen, dass man auf beiden Seiten sicher im vital spongiösen Knochen ist und die Beckenstärke berücksichtigt ist. Sofort nach der Knochenextraktion werden die Dübel eingebracht. d: es werden weitere Dübel eingebracht und ein Bohrdraht wird entfernt. e: der erste Bohrdraht wird durch eine Zugschraube ersetzt. f: zum Schluss wird der zweite Bohrdraht entfernt und durch eine Zugschraube ersetzt (Dresing und Stürmer 2000 S.648).

Eine Arthrodese kann arthroskopisch assistiert (AAA) durchgeführt werden. Diese Möglichkeit bietet im Vergleich zur "offenen“ Variante die Vorteile eines kürzeren Klinikaufenthaltes, eines geringeren Zugangstraumas, weniger Blutverlust, geringere Morbidität und zügigere Konsolidierung. Hierbei wird über einen posterolateralen Zugang der Gelenkknorpel reseziert. Dann wird mit einem kleinen Meißel der Knochen der korrespondierenden Seiten angefrischt. Von dorsal werden zwei Spongiosazugschrauben medial und lateral 
eingebracht. Die Fusionsrate im Vergleich zur offenen Operation ist gleich. Patienten mit starken Formabweichungen der Gelenkkonturen, erheblicher Rotationsfehlstellungen und neurogenen Arthropathien sollten offen operiert werden (Myerson und Quill 1991; Jerosch 2003).

\subsubsection{Die USG-Arthrodese}

Grundsätzlich sollte eine Versteifung des USG nur bei Beschwerden durchgeführt werden. Im Zweifel ist unter dem Bildwandler eine Lokalanästhetika-Infiltration durchzuführen (Fink 2005). Am unteren Sprunggelenk wird ausschließlich die interne Fixation angewandt (Stuhler 1994). Die Genese der therapierefraktären posttraumatischen Arthrose des USG ist mit der des OSG zu vergleichen. Ziel einer jeden USG-Arthrodese ist daher primär eine Schmerzreduktion für den Patienten. Darüber hinaus sollte eine anatomisch und biomechanisch gute Rekonstruktion stattfinden. Gerade in Bezug auf das Subtalargelenk ist auf die Rückfußachse (Varus/Valgus), die Rotation des Talus nach medial und die Vorfußabduktion mit einer Verkürzung der lateralen Fußsäule, die Talometatarsale Achse (Flexion des Talus nach dorsal) und die Entstehung eines lateralen „Bulge“ zu achten. Dies sind wichtige pathobiomechanische Prädiktoren für ein gutes postoperatives Ergebnis. Der Vorteil der reinen talokalkanearen Arthrodese liegt in dem Erhalt der Beweglichkeit vor allem im Chopart-Gelenk. Folglich bleibt dem Patienten eine Pro- und Supinationsfähigkeit im USG gut bis sehr gut erhalten. Bei der reinen Subtalar-Arthrodese wird, im Vergleich zur Double-Arthrodese, die OSGBeweglichkeit bewahrt. All diese Aspekte zeigen, dass für den Patienten ein besseres postoperatives Ergebnis geliefert wird, wenn eine Versteifung auf das symptomatische Gelenk konzentriert wird (Fellmann und Zollinger 1996; Thermann et al. 1999). Liegt eine posttraumatische Arthrose nach Kalkaneusfraktur oder Nekrose des Taluskorpus vor, so ist die Subtalararthrodese die Behandlung der Wahl. Der Vorteil bei diesem Verfahren ist, dass die Beweglichkeit im Chopart- Gelenk erhalten bzw. nur gering eingeschränkt wird. Das Risiko einer Anschlussarthrose ist dadurch reduziert 
(Fellmann und Zollinger 1996). Bei dieser Form werden zu Beginn die Gelenkknorpel reseziert und daraufhin der Sinus Tarsi mit Spongiosa aufgefüllt. Bei der späteren Fixation sollte auf eine korrekte Stellung des Rückfußes geachtet werden. Es hat sich eine 5-10 Valgisierung des Kalkaneus als sinnvoll herausgestellt. Fixiert wird der Kalkaneus dann mit Spongiosazugschrauben, die vom Kalkaneus in den Taluskorpus oder vom Talushals in den Kalkaneus eingebracht werden können (Trnka et al. 2001). Wenn nur das Talonavikulargelenk symptomatisch betroffen ist, so kann eine isolierte Talonavikulararthrodese helfen. Der Rückfuß sollte hierbei vorzugsweise in Neutralstellung fixiert sein. Der Gelenkspalt wird entknorpelt und mit Spongiosa aufgefüllt. Daraufhin wird das Gelenk mit 1-2 Zugschrauben mit distalem Gewinde fixiert (Fink 2005). Wenn alle 3 Rückfußgelenke arthrotisch destruiert sind, kommt eine komplexere, das Talokalkanear-, Talonavikular- und das Kalkaneokuboidal-Gelenk betreffende, sogenannte Triple-Arthrodese zum Einsatz. Indikationen hierfür ergeben sich nicht selten durch inflammatorische, angeborene, paralytische oder neuromuskuläre Erkrankungen. Häufig wird hierbei eine Zugschraubenarthrodese mit drei Schrauben durchgeführt. Eine Schraube verläuft vom Talus in den Kalkaneus, eine zweite vom Os naviculare in den Talus und eine dritte vom Os cuboideum in den Kalkaneus. Eine korrekte Position des Kalkaneus, vorzugsweise in 5-10- Valgisierung, muss beachtet werden. Die Arthrodese kann mit Klammern durchgeführt werden. Dadurch entsteht der Nachteil, dass keine Kompression in den Arthrodeseflächen aufgebaut wird (Fink 2005). Bei jüngeren Patienten und nicht betroffenem Subtalargelenk kann eine Double-Arthrodese durchgeführt werden. Diese entspricht der Triple-Arthrodese, nur dass hierbei das Subtalargelenk ausgespart bleibt (Fink 2005).

\subsection{Komplikationen}


Die Rate an Komplikationen ist bei internen im Vergleich zu externen Fixationsverfahren erheblich geringer ist (Moeckel et al. 1991; Thordarson et al. 1992; Zwipp et al. 1999; Buchner und Sabo 2003 b). Als Risiko aller Arthrodesen gilt jedoch primär die fehlende Konsolidierung. Es ist bekannt, dass das Rauchen in Bezug auf die Komplikationen meist einen erheblichen Schaden anrichten kann (Buchner und Sabo 2003 a).

Weitere Risiken können postoperative Infekte und Wundheilungsstörungen (426\%) sein. Pseudarthrosen (4-35\%) entstehen meist durch eine inadäquate Post-OP-Mobilisation. Weiter kann es zu Ermüdungsbrüchen, zum Entrapment (Popliteakompressionssyndrom) des N.tibialis bzw. der Gefäße mit Durchblutungsstörungen des Unterschenkels, Gangrän bzw. persistierende Ödeme oder schmerzhaften Neuromen kommen. Wesentlich zu erwähnen bleibt jedoch das Risiko von Anschlussarthrosen benachbarter Gelenke, die allerdings nicht immer klinisch manifest sein müssen. In der Literatur wird fast durchweg von guten bis sehr guten Langzeitergebnissen berichtet. Für gelungene Arthrodesen in Neutralstellung des Fußes werden sehr gute Ergebnisse in über $80 \%$ der Fälle angegeben.

\subsection{Nachbehandlung}

Im Allgemeinen kann gesagt werden, dass sich die Art der Nachbehandlung an der verwendeten Arthrodeseform orientieren sollte. Es gibt zwei Ansätze zur Nachbehandlung. Die einen präferieren die frühfunktionelle Mobilisation unter Entlastung bzw. Teilbelastung. Hier wird bei stabiler Arthrodese eine frühfunktionelle, gipsfreie Teilbelastung von $15 \mathrm{~kg}$ durchgeführt. Dann wird die Teilbelastung auf $30 \mathrm{~kg}$ erhöht und im Therapieschuh für etwa 6 Wochen durchgeführt. Bei schlechter Knochenqualität sollte die Zeit der Teilbelastung allerdings individuell angepasst werden (Thermann et al. 1999). Eine andere Variante ist hingegen die Gipsimmobilisation bzw. Teilbelastung für unter Umständen 6-12 Wochen (Zwipp und Grass 2005). Zwipp et al. (2005) empfiehlt bis zur Wundheilung das Tragen eines gut gepolsterten und 
gespaltenen Unterschenkelliegegipses. Danach kann auf das Tragen eines Unterschenkelgehgipsverbandes mit maximaler Belastung von $20 \mathrm{~kg}$ für etwa 6 Wochen gewechselt werden. Anschließend ist bei komplikationslosem Verlauf die Teilbelastung im Arthrodesestiefel bis etwa $20 \mathrm{~kg}$ bis zur 13. postoperativen Woche möglich. Täglich wird hierbei eine physiotherapeutische Manualtherapie v.a. des Chopart-Gelenks durchgeführt. Parallel wird ein Gehtraining und Muskelaufbau durchgeführt. Ab der 16. postoperativen Woche beginnt der Übergang in die Vollbelastung.

\section{Fragestellung}

Mit dieser Studie sollen zwei an der Klinik für Unfallchirurgie, Plastische und Wiederherstellungschirurgie der Georg-August-Universität zu Göttingen durchgeführte operative Sprunggelenks-Arthrodese-Verfahren verglichen werden. Hierbei sollte sich das Hauptaugenmerk auf die beiden häufigsten Arthrodesevarianten dieser Klinik, Knochen-Dübel-Arthrodese (KDA) und Resektions-Kompressions-Arthrodese, richten.

Für die Studie sollen präklinische und klinische Parameter genutzt werden, um u.a. anhand von Score-Ergebnissen eine vergleichende Aussage über die beiden Arthrodese-Varianten tätigen zu können. Um selektiv obere oder untere Sprunggelenksversteifungen gegeneinander vergleichen zu können, wurde das Kollektiv aufgeteilt.

Es sollte untersucht werden, ob ein Verfahren bessere Ergebnisse liefert, als das andere. Weiter sollte untersucht werden, ob mit den Verfahren an den Gelenken OSG und USG unterschiedliche Ergebnisse erzielt werden können.

\section{Material und Methode}

\subsection{Aufbau der Studie}


Es handelt sich um eine retrospektive Studie. Bei den eingeschlossenen Patienten wurde eine operative Versteifung an oberem oder unterem Sprunggelenk durchgeführt. Die Operationszeitpunkte erstrecken sich von 1994 bis 2007. Die Patienten wurden vornehmlich zu Hause besucht und klinisch untersucht. Nur im Einzelfall fand ein Empfang in der unfallchirurgischen Poliklinik der Universitätsklinik zu Göttingen statt.

\subsection{Patientenkollektiv}

Für die Studie wurden Patienten gewählt, die eine dauerhafte operative Sprunggelenksversteifung am OSG oder USG bekommen haben. Es wurden 73 Patienten angeschrieben. Dabei waren 22 (30,14\%) weibliche und 51 (69,86\%) männliche Personen vertreten. Von diesen haben 31 Patienten $(42,47 \%)$ eine isolierte OSG-Arthrodese erhalten, 29 eine isolierte USG-Arthrodese (39,73\%) und 13 Patienten (17,81\%) bekamen eine kombinierte OSG-/ USG-Arthrodese. Nach ausführlicher Adressenrecherche bis hin zu Nachfragen bei Hausarzt, Arbeitgeber oder Versicherungen, schickten 45 Patienten (61,64\%) einen ausgefüllten Fragebogen zurück. 6 Patienten (8,22\%) waren verstorben, 4 $(5,48 \%)$ waren nicht bereit an dieser Studie teilzunehmen, ein Patient war zur Kur abwesend und eine Patientin befand sich im Hospiz. 16 Patienten (21,92\%) waren unbekannt verzogen. Von den 45 Patienten, die den Fragebogen zurück sendeten, wurde mit 40 Personen telefonisch ein Untersuchungstermin vereinbart. 5 Patienten wurden aufgrund von einer Entfernung $>300$ km nicht untersucht.

Unter diesen 40 Personen waren 35, die entweder eine isolierte OSG oder USG Arthrodese bekommen haben.

\subsection{Auswertung von Krankenakten}


Alle 73 Patientenakten standen zur Einsicht zur Verfügung. Die hierin enthaltenen Daten wurden mit dem Programm FileMaker Pro 8.5v1 (c) dargestellt und ausgewertet.

\subsection{Erstellen und Versenden des Fragebogens}

Für die Evaluation wurde ein Fragebogen selbst entwickelt, der sich u.a. auch (siehe Anhang) an 3 bekannten Scores (siehe 4.6) orientiert. Damit ist eine spätere Vergleichbarkeit gewährleistet. Im Grundsatz wurden daher Fragen zu folgenden Bereichen formuliert:

- Schmerz

- Subjektive Zufriedenheit

- Gehhilfen

- Gehstrecke

- Schmerzmitteleinnahme

- Zehenspitzenstand

- Treppensteigen

- Gehprobleme.

Bei diesem Fragebogen handelt es sich ausschließlich um subjektive Daten. Die objektiven Daten wurden durch die klinische Untersuchung gewonnen (siehe 4.5). Den Patienten wurde ein Brief mit einem aufklärenden Anschreiben zum Zweck dieser Studie sowie der Bitte um Teilnahme zu gesendet. Ein frankierter Rückumschlag und der Fragebogen wurden beigefügt. Den Patienten wurde mitgeteilt, dass die Teilnahme freiwillig sei und die Daten pseudonymisiert würden. Die Daten der zurück gesendeten Fragebögen wurden in die oben angegebene Datenbank übernommen. 


\subsection{Klinische Untersuchung der Patienten}

Die Patienten wurden telefonisch kontaktiert und wie im Fragebogen auf die Freiwilligkeit hingewiesen. Hierbei wurden folgende Aspekte klinisch untersucht:

- Dorsalextension und Plantarflexion des Fußes nach der „Neutral-0Methode" bzw. die Stellung des Fußes zum Unterschenkel

- Achseneinstellung zwischen Unterschenkel und Rückfuß (mit der Frage nach Valgisierung)

- Rückfußbeweglichkeit (hierbei wurde der Patient gebeten barfuß zu gehen, dabei wurde die Beweglichkeit des Rückfußes zwischen beiden Füßen verglichen)

- Gangbild (mit der Frage nach eventuellem Hinken oder anderen Auffälligkeiten).

Darüber hinaus wurde mit dem Patienten über subjektive Probleme gesprochen. Es sollten Angaben zum Schmerz und zur persönlichen Zufriedenheit mit den im Fragebogen angegebenen Aussagen abgeglichen werden.

\subsection{Score}

Es wurden die 3 international anerkannten Scores von Kitaoka et al. (1994) (Ankle-Hindfoot- Score), Mazur et al. (1979) (Boston children's Hospital ankle scoring system) und von McGuire et al. (1988) angewendet, die bei dem Thema der Sprunggelenksarthrodese breite Verwendung finden (1988; Helm 1990; Schaap et al. 1990; Rehart et al. 1999; Bonnaire et al. 2001; Schulze et al. 2002; Horn 2003; Kennedy et al. 2003; Lauge-Pedersen 2003).

Anhand dieser drei Scores konnten die Ergebnisse dargestellt werden. An innen wird die postoperative Situation auf einer Scala von 0-100 Punkten dargestellt, von „schlecht” bis „sehr gut“. 


\subsubsection{Ankle-Hindfoot Score}

Der Ankle-Hindfoot-Score ist ein von der American Orthopaedic Foot and Ankle Society empfohlener Score mit dem Ziel Schmerzen, Funktionen und Achsenverhältnisse des Fußes bzw. der Sprunggelenke evaluieren zu können. Es ist ein klinischer Score, der sich als valides Instrument bei der Bewertung von Sprunggelenksarthrodesen etabliert hat. Dieser Score beinhaltet zu 60\% subjektive und zu 40\% objektive Daten, wobei der Schmerz am höchsten gewichtet wird (Abb.9).

Kriterien in diesem Score sind:

- Schmerz (45\%)

- Aktivität/Gehhilfe (10\%)

- Gehstrecke (10\%)

- Hinken (10\%)

- Rückfußbewegung (10\%)

- Achsenverhältnisse (15\%).

(Kitaoka et al. 1994; Hildebrand et al. 1996; Schuh und Hausel 2000) 


\begin{tabular}{|c|c|}
\hline AOFASAnkle-Hindfoot Scale (100 Punkte) & Punkt \\
\hline \multicolumn{2}{|l|}{ Schmerz (40 Punkte) } \\
\hline Keiner & 40 \\
\hline Leicht, gelegentlich & 30 \\
\hline Mäßig, täglich & 20 \\
\hline Schwer, fast immer präsent & 0 \\
\hline \multicolumn{2}{|l|}{ Funktion (50 Punkte) } \\
\hline \multicolumn{2}{|l|}{ Tägliche Einschränkungen, Notwendigkeit von Unterstützung } \\
\hline Keine Einschränkung, keine Unterstützung & 10 \\
\hline Keine Einschränkung von täglichen Aktivitäten, & 7 \\
\hline \multicolumn{2}{|l|}{ Einschränkung von Freizeitaktivitäten, keine Unterstützung } \\
\hline Einschränkung von täglichen und Freizeitaktivitäten, Stock & 4 \\
\hline $\begin{array}{l}\text { Schwere Einschränkung von täglichen und Freizeitaktivitäten, Gehwagen, Krücken, } \\
\text { Rollstuhl, Brace }\end{array}$ & 0 \\
\hline \multicolumn{2}{|l|}{ Maximale Gehstrecke (Häuserblocks) } \\
\hline$>6$ & 5 \\
\hline $4-6$ & 4 \\
\hline $1-3$ & 2 \\
\hline$<1$ & 0 \\
\hline \multicolumn{2}{|l|}{ Gehen auf Oberflächen } \\
\hline Keine Schwierigkeiten auf allen Oberflächen & 5 \\
\hline Etwas Probleme auf unebenen Flächen, Stufen, Leitern, Neigung & 3 \\
\hline Starke Probleme auf unebenen Flächen, Stufen, Leitern, Neigung & 0 \\
\hline \multicolumn{2}{|l|}{ Gangbildstörungen } \\
\hline Keine, leichte & 8 \\
\hline Auffällige & 4 \\
\hline Starke & 0 \\
\hline \multicolumn{2}{|l|}{ Sagitale Bewegung (Flexion/Extension) } \\
\hline Normal oder leichte Einschränkung $\left(\geqq 30^{\circ}\right)$ & 8 \\
\hline Mäßiggradige Einschränkung $\left(15^{\circ}-29^{\circ}\right)$ & 4 \\
\hline Starke Einschränkung $\left(<15^{\circ}\right)$ & 0 \\
\hline \multicolumn{2}{|l|}{ Rückfußbeweglichkeit ( Inversion plus Eversion) } \\
\hline Normal oder leichte Einschränkung ( $75-100 \%$ des Normalen ) & 6 \\
\hline Mäßiggradige Einschränkung ( $25-74 \%$ des Normalen ) & 3 \\
\hline Starke Einschränkung ( $<25 \%$ des Normalen) & 0 \\
\hline \multicolumn{2}{|l|}{ Sprunggelenk-Rückfußstabilität (a.-p., valgus-varus) } \\
\hline Stabil & 8 \\
\hline Deutlich instabil & 4 \\
\hline \multicolumn{2}{|l|}{ Form,Alignement (10 Punkte) } \\
\hline Gut, „Sohlengänger-Fuß", OSG-Rückfußachse gut & 10 \\
\hline $\begin{array}{l}\text { Mäßig, „Sohlengänger-Fuß“, geringe Abweichung der OSG-Rückfußachse, } \\
\text { keine Symptome }\end{array}$ & 5 \\
\hline Schlecht, kein "Sohlengängerfuß", schwere Achsabweichung, Symptome & 0 \\
\hline
\end{tabular}

Abb. 9: Ankle-Hindfoot Score (Schuh und Hausel 2000 S.297) 


\subsubsection{Mazur Score}

Der Mazur-Score verwendet objektive und subjektive Daten. Anwendung findet dieser v.a. bei Sprunggelenksarthrodesen. Hier werden jeweils 50 Punkte für die Beschwerdesymptomatik und für die Funktion verteilt, so dass maximal 100 Punkte zu erreichen sind. Im Einzelnen wird hier nach Folgendem gefragt:

- Schmerz (50\%)

- Hinken (6\%)

- $\quad$ Trepp rauf und runter (6\%)

- Steigung hoch und runter (6\%)

- Zehenspitzenstand (5\%)

- Laufen (5\%)

- Gehstrecke (6\%)

- Gehhilfe (6\%)

- Dorsalextension, Plantarflexion (10\%)

(Mazur et al. 1979).

\subsubsection{McGuire et al. Score}

Der McGuire et al. Score ist ein klinisch ausgerichteter Score, der zu 95\% aus subjektiven und zu 5\% aus objektiven Fragen besteht. Er bezieht sich auf das obere Sprunggelenk.

Folgende Bereich werden hier befragt:

- Schmerz (50\%)

- Bewegungsausmaß (Dorsalextension, Plantarflexion des Fußes) 5\%

- Gehstrecke (20\%)

- Treppensteigen (10\%)

- Gehhilfen (5\%)

- Hinken (5\%)

- Unebene Gehstrecke (5\%)

(McGuire et al. 1988). 


\subsection{Dokumentation, Statistik und Auswertung}

Sämtliche Daten aus den Patientenakten, den Fragebögen und der klinischen Untersuchung wurden in die relationale Datenbank FileMaker Pro 8.5v1@ eingegeben. Die Masken wurden eigenständig programmiert. Mit dieser können beliebige Daten zu Patienten jeweils beliebig zueinander ins Verhältnis gesetzt und dargestellt bzw. ausgewertet werden. Alle Analysen wurden mit der Software Statistica (Version 8.0, StatSoft) vorgenommen.

Bei der statistischen Auswertung wurden kategorielle Variablen zwischen den Operationsgruppen bzw. den Spunggelenksstellen mit dem Chi-Quadrat-Test verglichen. Die ordinalen und metrischen Daten wurden mit dem MannWhitney-U-Test analysiert. Die kategoriellen Ergebnisse wurden prozentual und mit Hilfe von Balkendiagrammen dargestellt. Die Scorewerte und die Altersverteilungen wurden durch Boxplots präsentiert. Als Signifikanzniveau wurde Alpha $=5 \%$ festgelegt.

\section{Ergebnisse}

\subsection{KDA versus Kompressionsarthrodese unabhängig vom Gelenk}

Für die statistische Auswertung wurden aus dem Pool von 40 Patienten diejenigen ausgewählt, die entweder eine KDA-Arthrodese oder aber eine Resektions-, Kompressionsarthrodese erhalten haben. Insgesamt sind dies 35 Patienten. 24 Männern (69\%) stehen 11 Frauen (31\%) gegenüber. Hiervon sind $24(68,6 \%)$ mit einer KDA-Arthrodese und $11(31,4 \%)$ mit einer Resektions-, Kompressionsarthrodese versorgt worden. 21 Patienten (60\%) bekamen eine OSG-Arthrodese und 14 (40\%) eine USG-Arthrodese. Von den 24 KDA-Arthrodesen waren 13 (54,2\%) OSG-Versteifungen, während bei 11 Patienten $(45,8 \%)$ das USG versteift wurde. 
$8(72,7 \%)$ der 11 Resektions-, Kompressionsarthrodesen betrafen das OSG und bei $3(27,3 \%)$ Patienten wurde auf diese Weise das USG versteift. Von den 24 KDA Patienten waren 18 männlich (75\%) und 6 weiblich (24\%). Unter der Kompressions-Arthrodese waren 6 männliche (54,5\%) und 5 weibliche (45,5\%) Patienten.

\subsubsection{Altersverteilung}

KDA-Kollektiv

Das Durchschnittsalter der Patienten in der KDA-Gruppe lag bei $42,65 \pm 10,98$ [20-61] Jahren mit einem Median von 41,66 Jahren.

\section{Kompressions-Arthrodese-Kollektiv}

Das durchschnittliche Alter in der Gruppe der Kompressions-Arthrodese lag bei 48,19 $\pm 16,60$ [20-74] Jahren mit einem Median bei 46,69 Jahren.

Im Vergleich zur KDA-Gruppe sind die Patienten mit Kompressions-Arthrodese etwas älter, was jedoch nicht signifikant ist. 


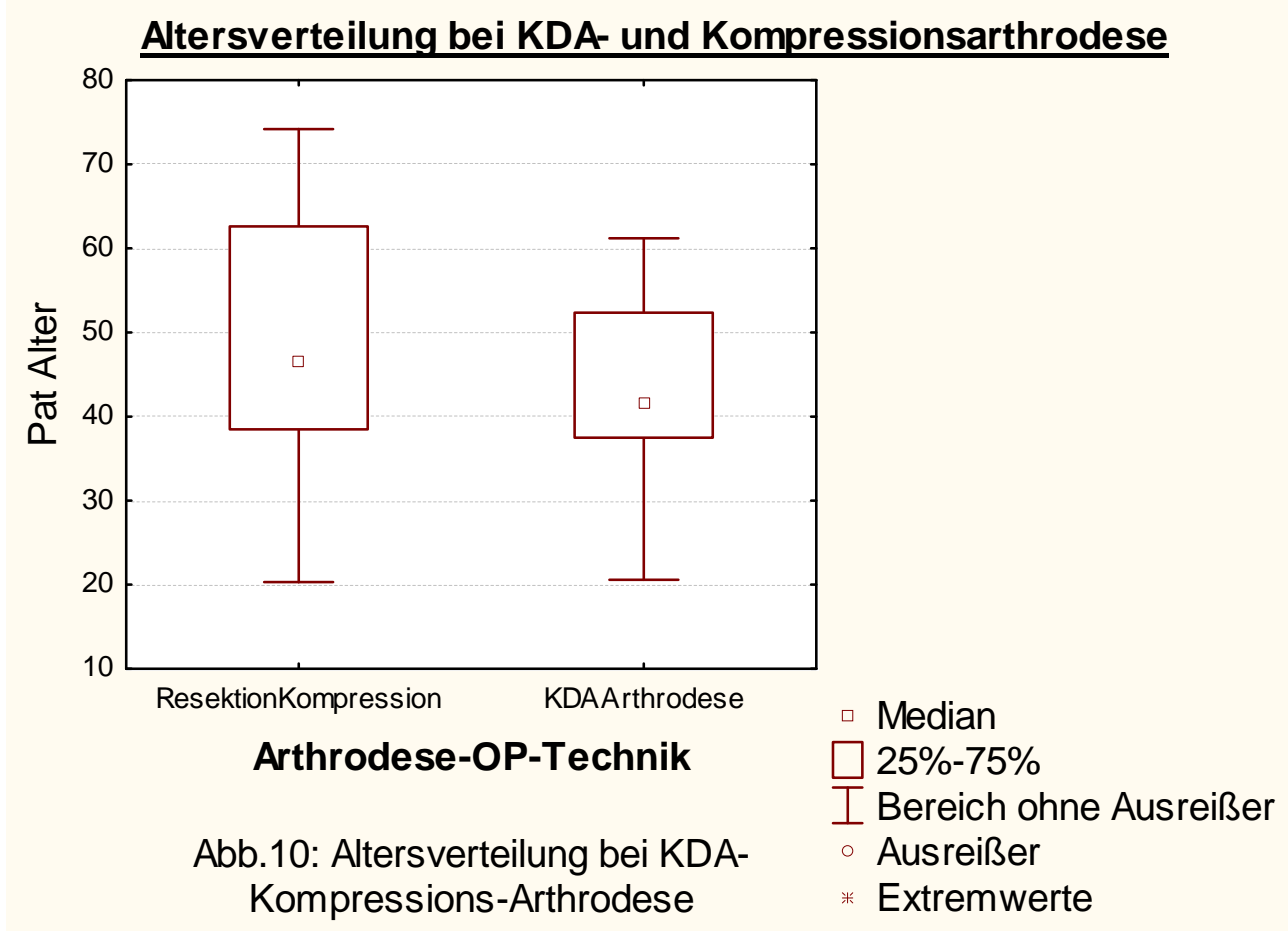

\subsubsection{Score-Ergebnisdarstellung}

\section{KDA-Kollektiv}

Die durchschnittlichen Score Werte lagen bei dem KDA-Kollektiv beim KitaokaScore bei 67,54 $\pm 20,43$ [29-100] Punkten, beim McGuire-Score bei 71,13 $\pm 15,76$ [37-99] Punkten und beim Mazur-Score bei 53,92 21,64 [11-89] Punkten.

\section{Kompressions-Arthrodese-Kollektiv}

Bei der Resektions-,Kompressionsarthrodese, im Folgenden nur KompressionsArthrodese genannt, lag der durchschnittliche Kitaoka-Score bei 63,36 $\pm 15,65$ [38-85] Punkten, der durchschnittliche McGuire-Score bei 65,64 $\pm 10,87$ [48-82] Punkten und der durchschnittliche Mazur-Score lag bei 50,73 $\pm 16,59$ [20-73] Punkten.

Hier erreichen alle drei Scores bei der KDA-Gruppe etwas höhere Punktzahlen, als bei den mit einer Kompressions-Arthrodese versorgten Patienten. Dies ist jedoch nicht signifikant. 

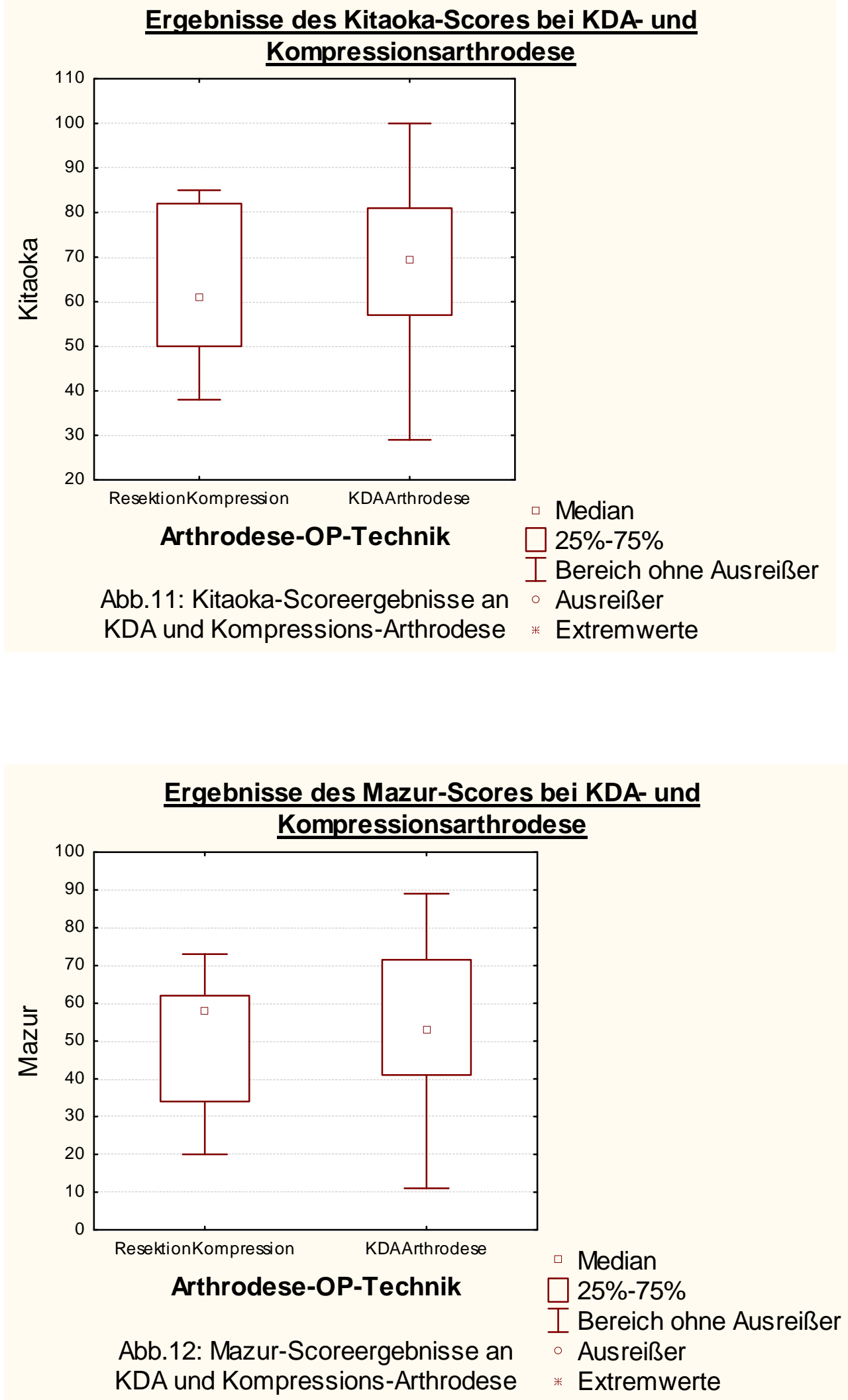


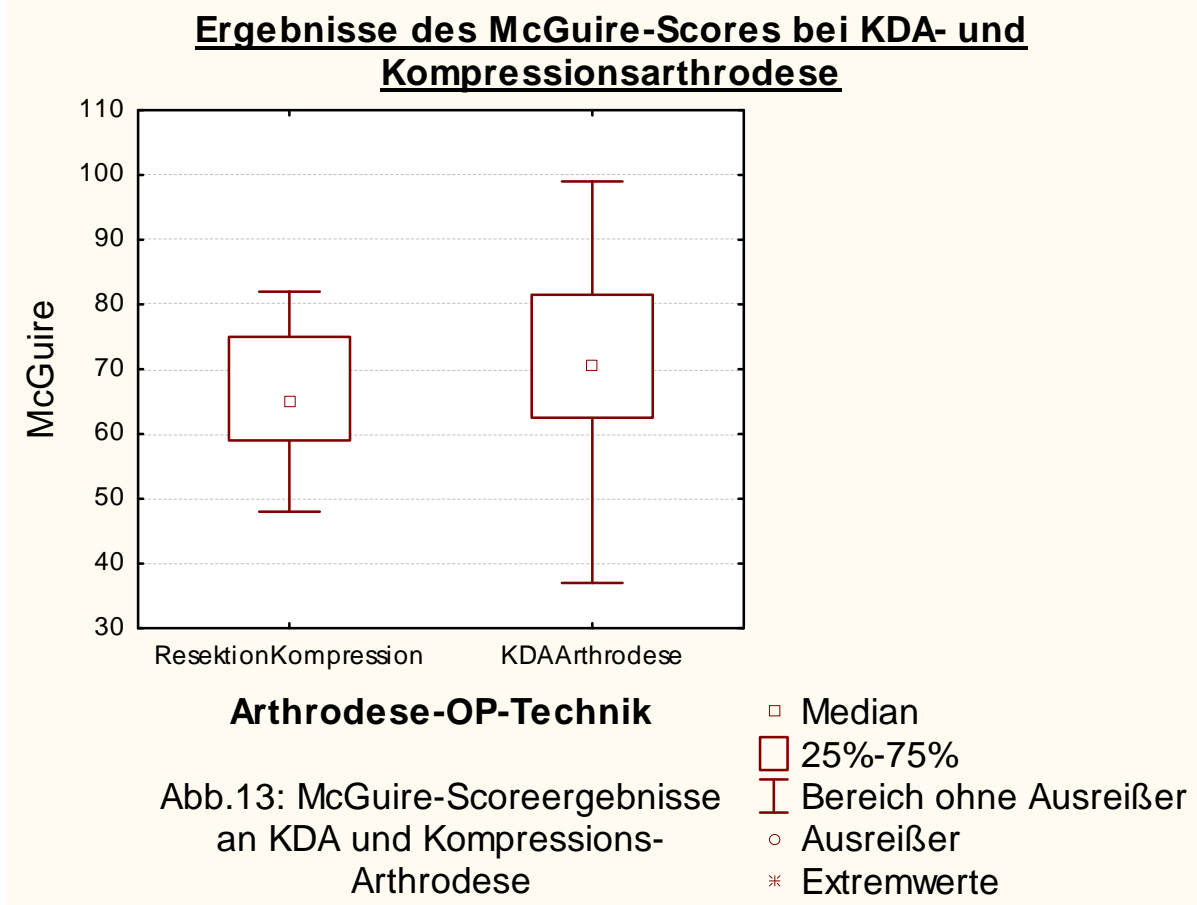

\subsubsection{Primäre Diagnosen}

\section{KDA-Kollektiv}

5 Patienten (20,83\%) hatten primär eine OSG-Fraktur. Eine Pilon-tibiale Fraktur hatten 8 Patienten (33,33\%). Eine Kalkaneusfraktur wurde primär bei 10 Patienten (41,67\%) diagnostiziert. 1 Patient hatte eine Talusfraktur.

\section{Kompressions-Arthrodese-Kollektiv}

Hier hatte ein Patient eine OSG-Fraktur. 5 Patienten (45,45\%) kamen mit einer primären Pilon-tibiale Fraktur. Eine Kalkaneusfraktur hatten ursprünglich 2 Patienten. Ein Patient hatte eine Talusfraktur. Eine traumatische Fraktur einer vorbestehenden Arthrodese passierte bei einem Patienten. Ein weiterer Patient kam primär mit einer Pilon-tibiale- und Kalkaneusfraktur. 


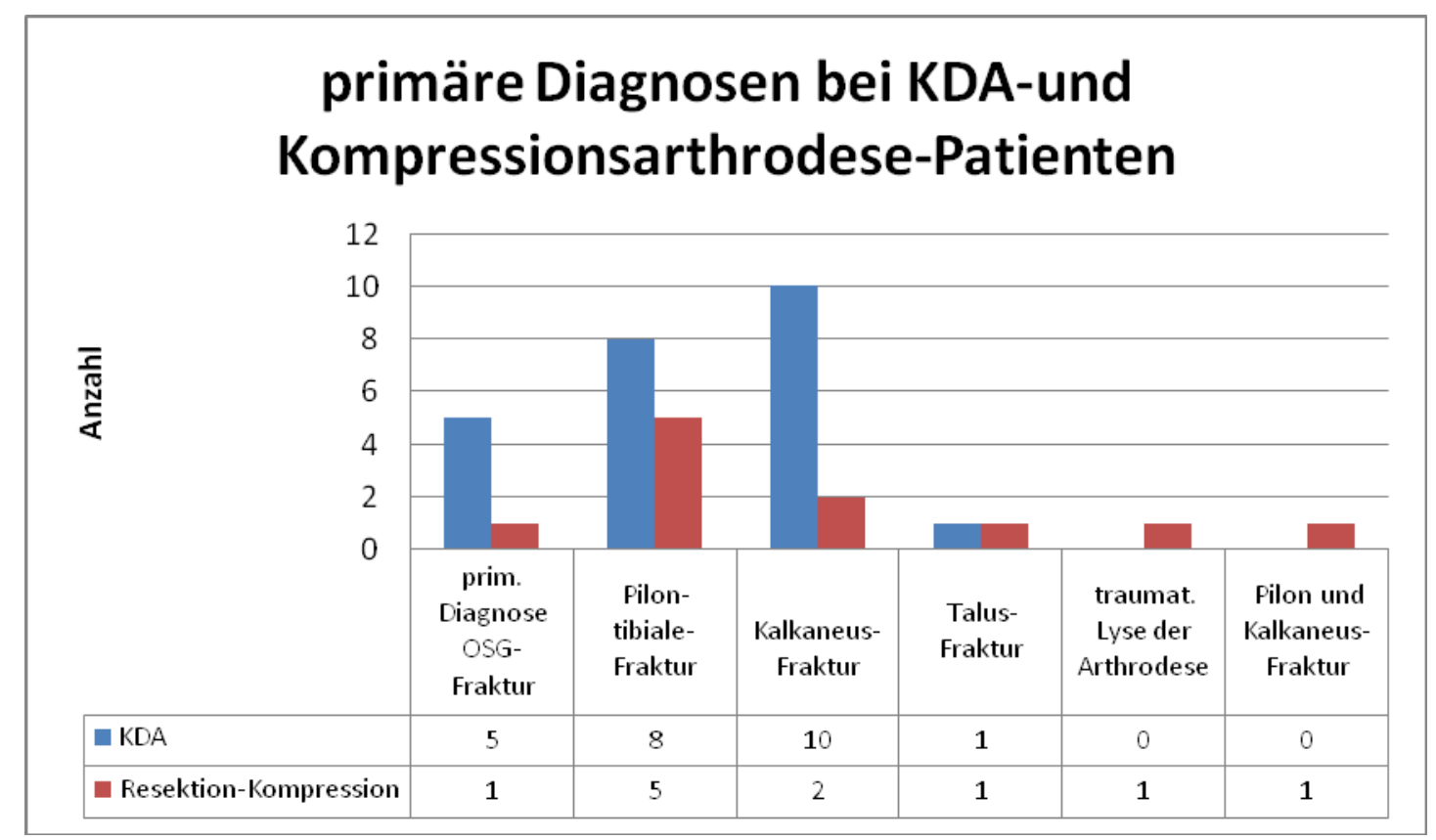

Abb.14: primäre Diagnosen bei KDA und Kompressions-Arthrodese-Patienten

\subsubsection{Operationsindikationen}

\section{KDA-Kollektiv}

2 Patienten wurden aufgrund einer Pseudarthrose operiert. Wegen einer

Talusnekrose wurde bei einem Patienten die Operationsindikation gestellt. Eine exazerbierte Entzündung war bei einem Patienten der Grund für eine Arthrodese. Bei 20 Patienten (83,33\%) war eine therapieresistente Schmerzsymptomatik die Indikation zur Gelenkversteifung.

\section{Kompressions-Arthrodese-Kollektiv}

Bei 6 Patienten (54,55\%) war eine Pseudarthrose die Operationsindikation zur Arthrodese. Eine exazerbierte Entzündung war Indikation zur Versteifung bei einem Patienten. Die Indikation zur Arthrodese wurde bei 4 Patienten (36,36\%) aufgrund einer therapieresistenten Schmerzsymptomatik gestellt. 


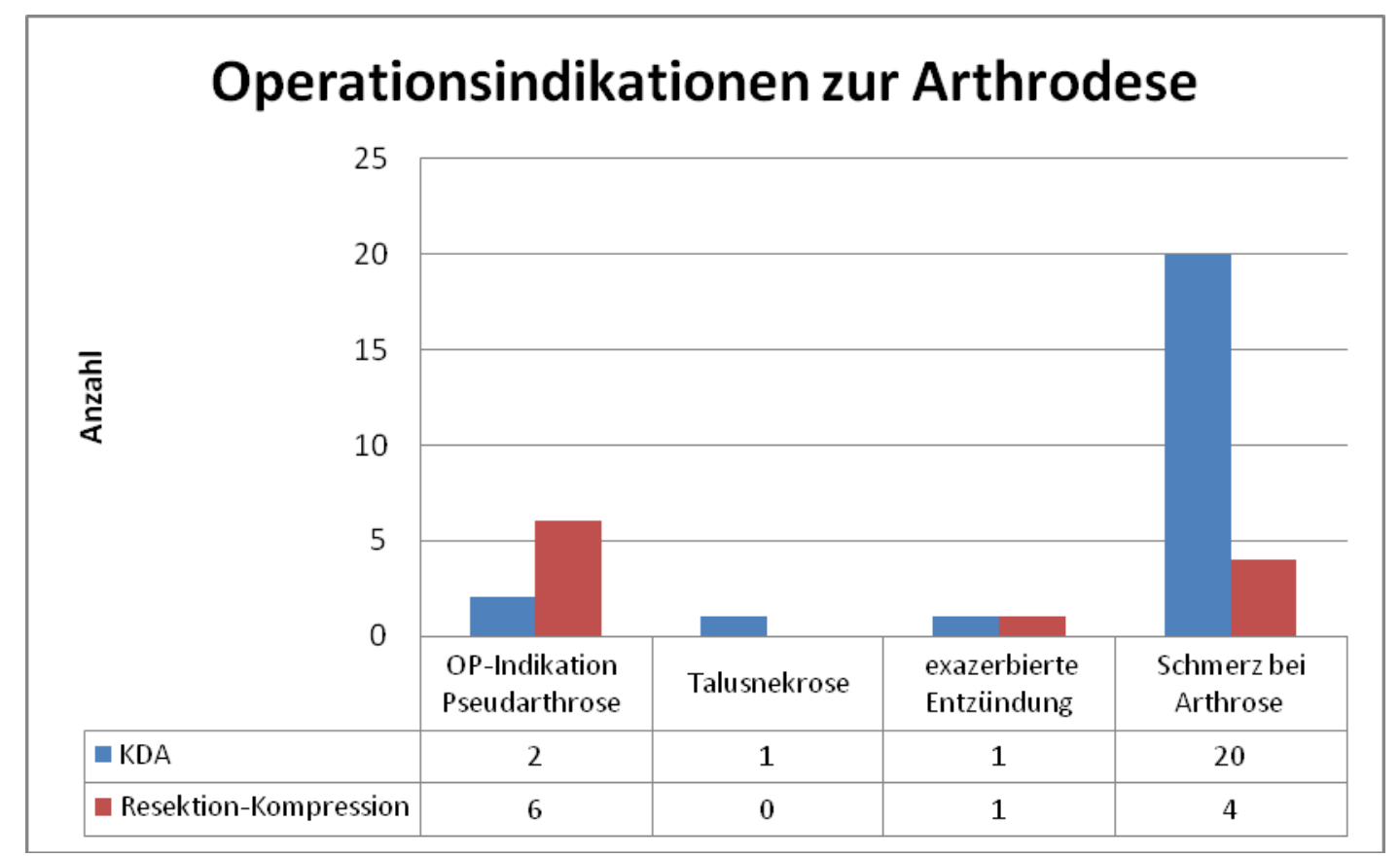

Abb.15: Operationsindikationen zur Arthrodese

\subsubsection{Perioperative Komplikationen und Spätfolgen}

In dieser Rubrik ist eine Differenzierung von perioperativen Komplikationen und Spätfolgen notwendig. Die perioperativen Komplikationen (Infekt, u.U.

Schraubenbruch) ereignen sich während des Krankenhausaufenthaltes bzw. bis maximal kurz nach der Entlassung. Die Spätfolgen (Anschlussarthrosen, Schraubenbruch oder Infekt in Form einer chron. Osteomyelitis etc.) sind erst nach vielen Monaten bis Jahren zu finden.

\section{KDA-Kollektiv}

14 Patienten (58,33\%) hatten keine Probleme bzw. Komplikationen. Eine OSGAnschlussarthrose entwickelten 3 Personen, die zuvor eine USG-Arthrodese bekommen haben. Eine Anschlussarthrose am USG bekamen 2 Patienten, die initial mit einer OSG-Arthrodese versorgt wurden. Bei einem Patienten gab es einen Schraubenbruch zwei Jahre nach der Arthrodese. 
Eine Anschlussarthrose in den Tarsometatarsalgelenken bekamen 2 Patienten. Ein Patient entwickelte nach zwei Jahren ein Peronealsehnenimpingement. Einen lokalen Infekt mit Abszessbildung gab es bei einem Patienten noch während des Krankenhausaufenthaltes.

\section{Kompressions-Arthrodese-Kollektiv}

Bei 5 Patienten $(45,45 \%)$ gab es keine Komplikationen oder Folgeprobleme. Ein Patient hatte einen Schraubenbruch nach einem Jahr. Eine Anschlussarthrose in den Tarsometatarsalgelenken trat bei 2 Patienten auf. 3 Patienten entwickelten postoperativ einen lokalen Infekt.

Ein signifikanter Unterschied war zwischen beiden Gruppen nicht zu ermitteln.

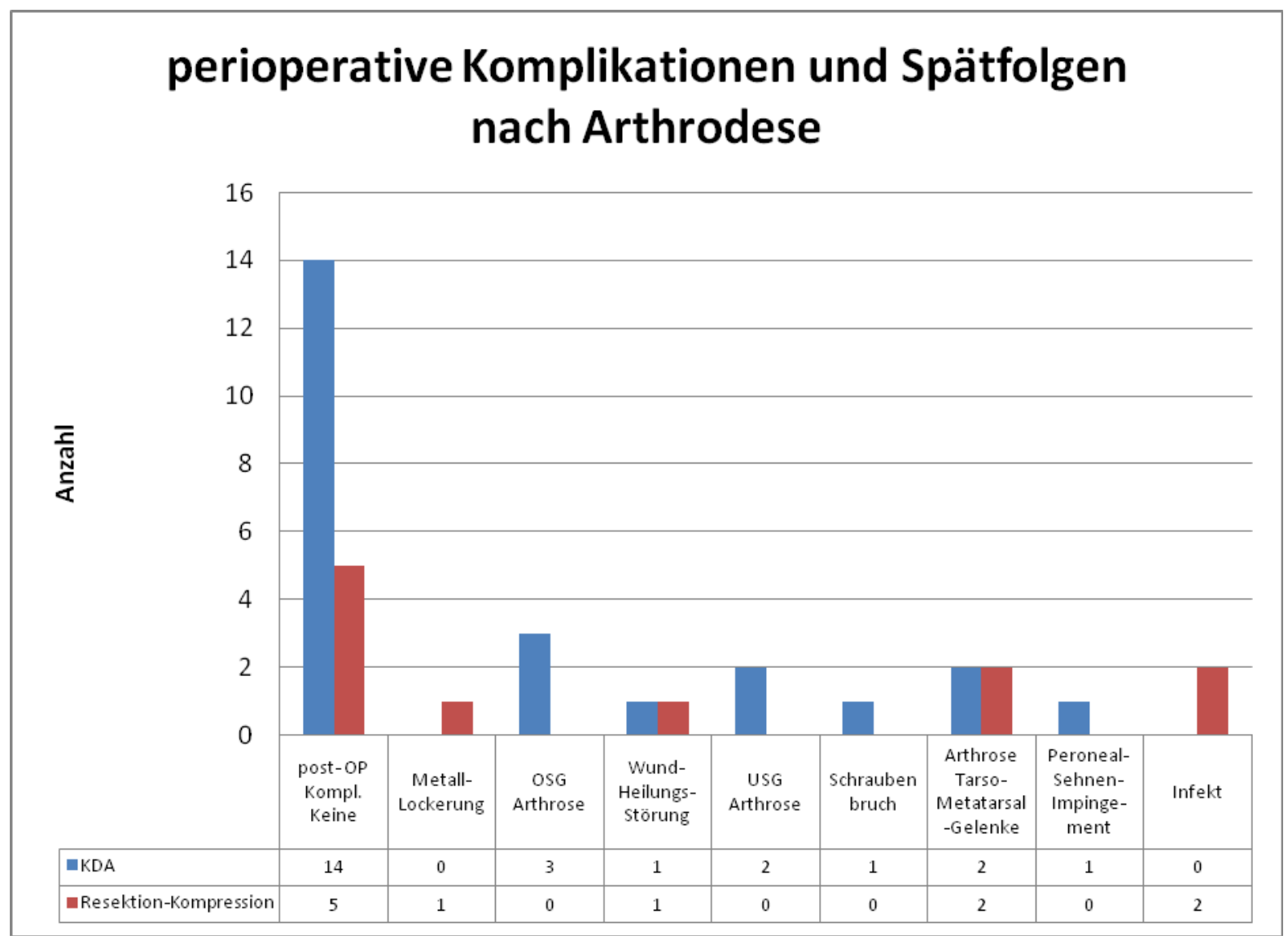

Abb.16: perioperative Komplikationen und Spätfolgen 


\subsubsection{Dauerhafte Schmerzen nach OP}

\section{KDA-Kollektiv}

3 Patienten gaben an seit der Versteifung überhaupt keine Schmerzen mehr zu haben. Über nur leichte Schmerzen klagen 8 Patienten (33,33\%). Erträgliche Schmerzen haben 5 Patienten (20,83\%). 4 Patienten (16,67\%) haben noch starke Schmerzen. Über Anlaufschmerzen berichten 4 Patienten (16,67\%).

\section{Kompressions-Arthrodese-Kollektiv}

Hier gibt es keinen Patienten, der über eine seit der Versteifung bestehende Schmerzfreiheit berichten kann. 4 Patienten (36,36\%) haben hingegen seit dem leichte Schmerzen. Weitere 3 Patienten berichten über erträgliche Schmerzen seit der Versteifung. Ein Patient hat starke Schmerzen. Über Anlaufschmerzen berichten 3 Patienten.

Ein statistisch signifikanter Unterschied ist nicht zu beobachten.

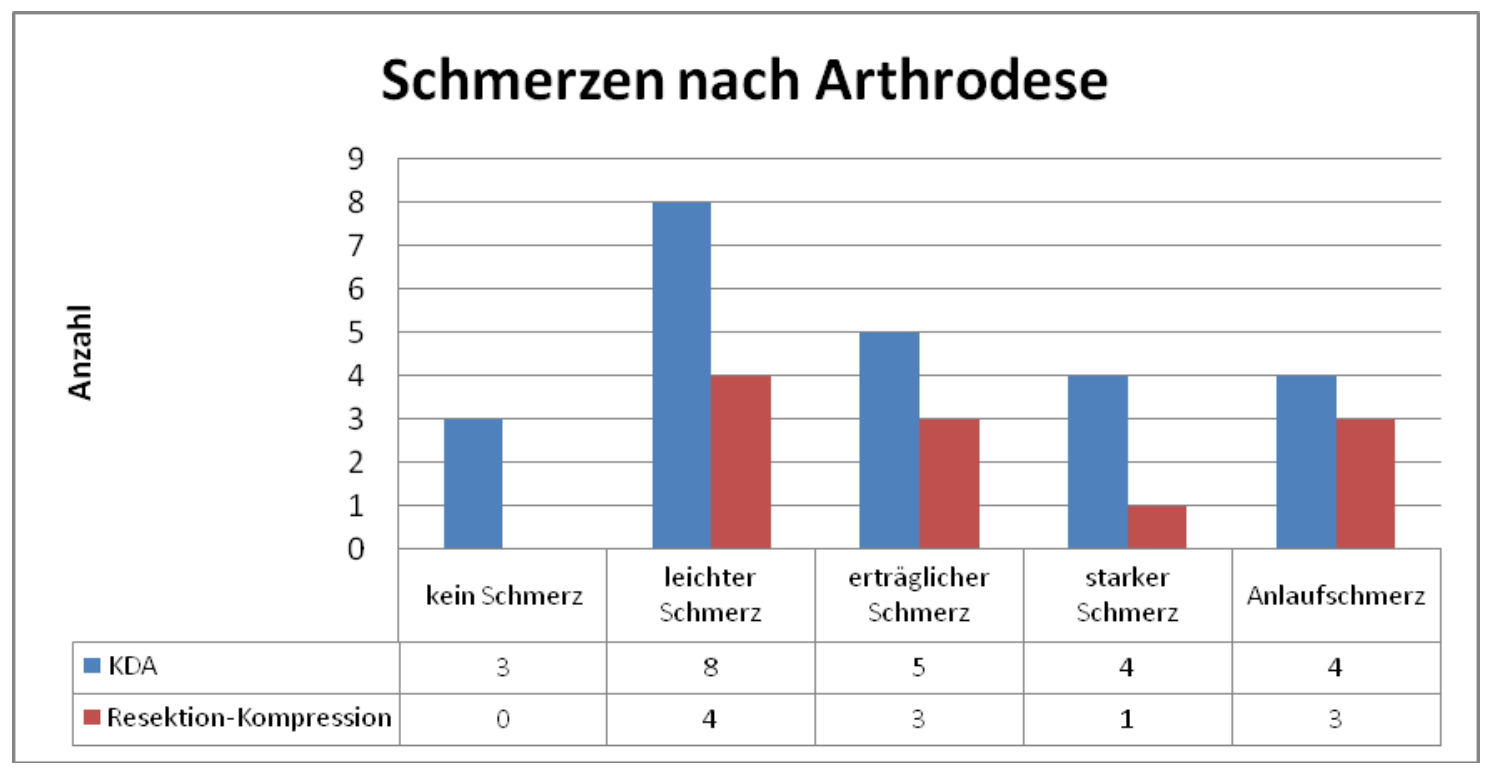

Abb.17: Schmerzen 


\subsubsection{Belastungsabhängige Schmerzen nach OP}

\section{KDA-Kollektiv}

4 Patienten $(16,67 \%)$ geben an unter Belastung keine Schmerzen zu haben.

Gelegentlich Schmerzen unter Belastung haben hingegen 10 Patienten

(41,67\%). Unter kontinuierlichen Schmerzen unter Belastung klagen 4

Personen (16,67\%). Ruhe- oder Spontanschmerz haben 6 Patienten (25\%).

\section{Kompressions-Arthrodese-Kollektiv}

Hier gibt es keinen Patienten, der unter Belastung schmerzfrei ist.

Gelegentliche Schmerzen unter Belastung haben 6 Personen (54,55\%). 3

Patienten geben an unter Belastung kontinuierliche Schmerzen zu haben.

Ruhe- oder Spontanschmerz haben 2 Patienten.

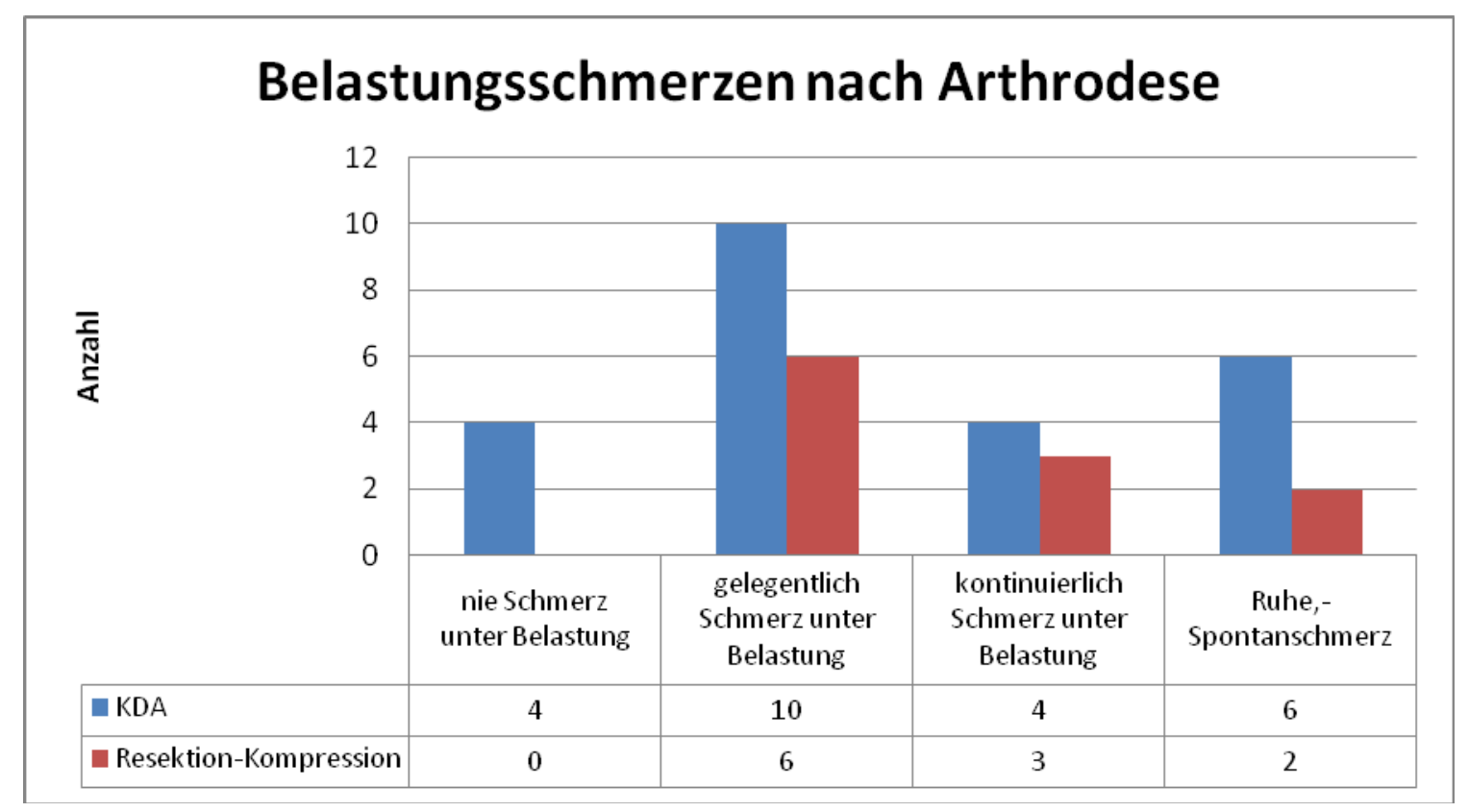

Abb.18: Belastungsschmerzen 


\subsubsection{Dauerhafte Schmerzmitteleinnahme nach OP}

\section{KDA-Kollektiv}

14 Patienten $(58,33 \%)$ nehmen keine Schmerzmittel ein. 1 bis 2 Mal pro Woche nehmen 5 Patienten (20,83\%) Schmerzmittel. 2 Personen geben an 3 bis 5 Mal pro Woche Schmerzmittel zu nehmen. Über eine tägliche Schmerzmitteleinnahme berichten 3 Patienten.

\section{Kompressions-Arthrodese-Kollektiv}

Hier geben 3 Patienten an, niemals Schmerzmittel nehmen zu müssen. 4 Personen (36,36\%) berichten über eine Schmerzmitteleinnahme 1 bis 2 Mal pro Woche. 3 bis 5 Mal pro Woche nehmen zwei Patienten Schmerzmittel ein. Zwei Personen berichten über eine tägliche Schmerzmitteleinnahme.

Hier sind keine signifikanten Ergebnisse zu sehen.

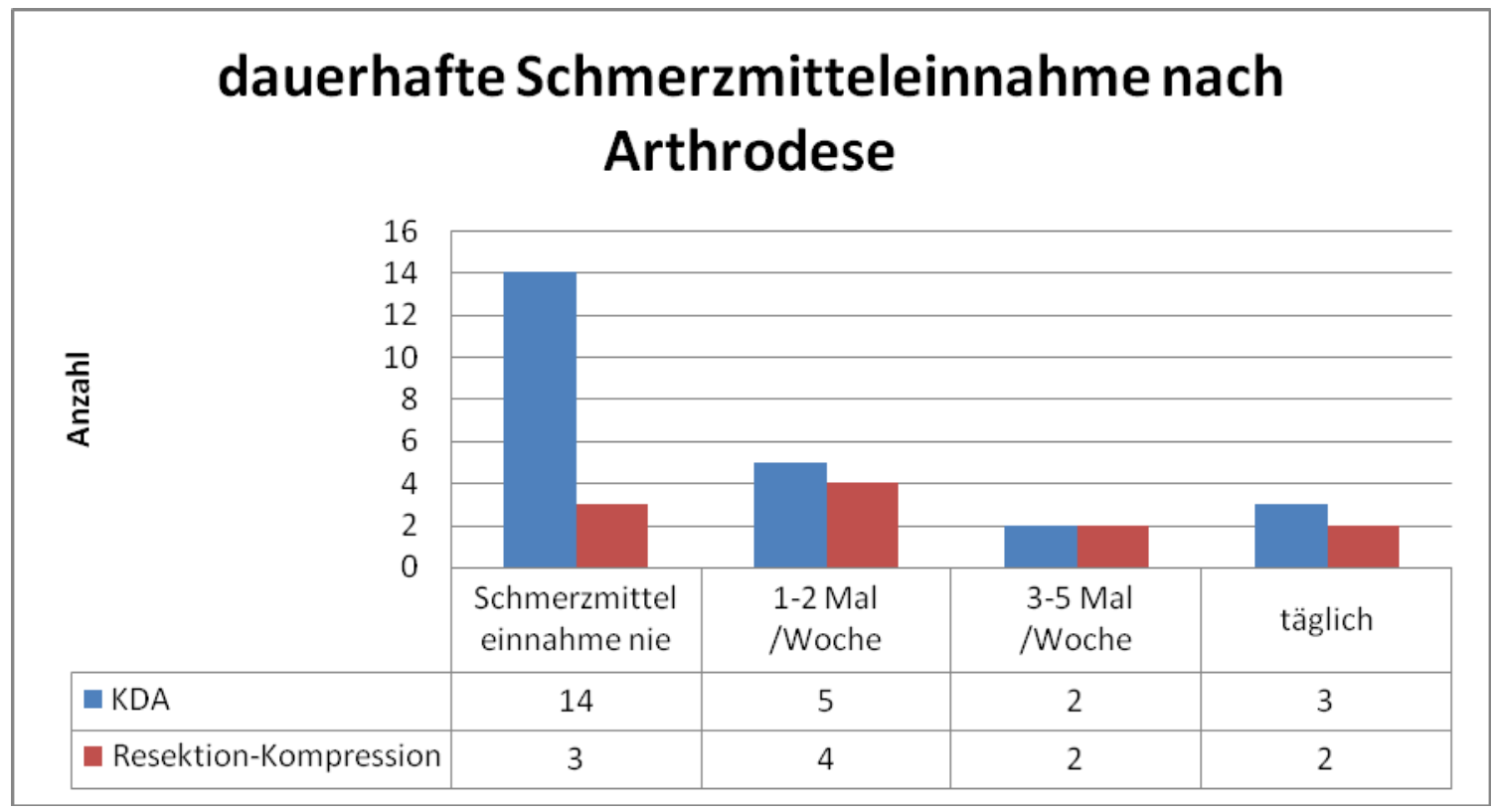

Abb.19: dauerhafte Schmerzmitteleinnahme 


\subsubsection{Schmerzfreie Gehstrecke nach OP}

\section{KDA-Kollektiv}

Eine schmerzfreie Gehstrecke ohne Einschränkungen konnten 4 Patienten $(16,67 \%)$ zurücklegen. 5 Personen (20,83\%) geben an 2 Stunden oder 8 bis 10 km gehen zu können ohne Schmerzen zu haben. Ebenfalls 5 Patienten $(20,83 \%)$ sagen, sie können 1 Stunde gehen ohne Schmerzen zu bekommen. Eine schmerzfreie Gehstrecke von etwa 30 Minuten oder 1-2 km konnten 2 Patienten zurücklegen. Ein Patient gibt an 15 Minuten ohne Schmerzen gehen zu können. Nur in Etappen mit Pausen schmerzfrei gehen zu können, geben 7 Personen $(29,17 \%)$ an.

\section{Kompressions-Arthrodese-Kollektiv}

Im Kompressions-Arthrodese-Kollektiv gibt es keinen Patienten, der ohne Einschränkungen schmerzfrei gehen kann. Ein Patient kann 2 Stunden schmerzfrei gehen. Ein weiterer Patient kann maximal eine Stunde schmerzfrei gehen. 30 Minuten ohne Schmerzen können 2 Personen gehen. Hier sind 6 Patienten (54,55\%), die ein schmerzfreies Gehen nur in Etappen mit Pausen erreichen.

Eine Signifikanz lässt sich hier trotz besserer Ergebnisse in der KDA-Gruppe nicht nachweisen. 


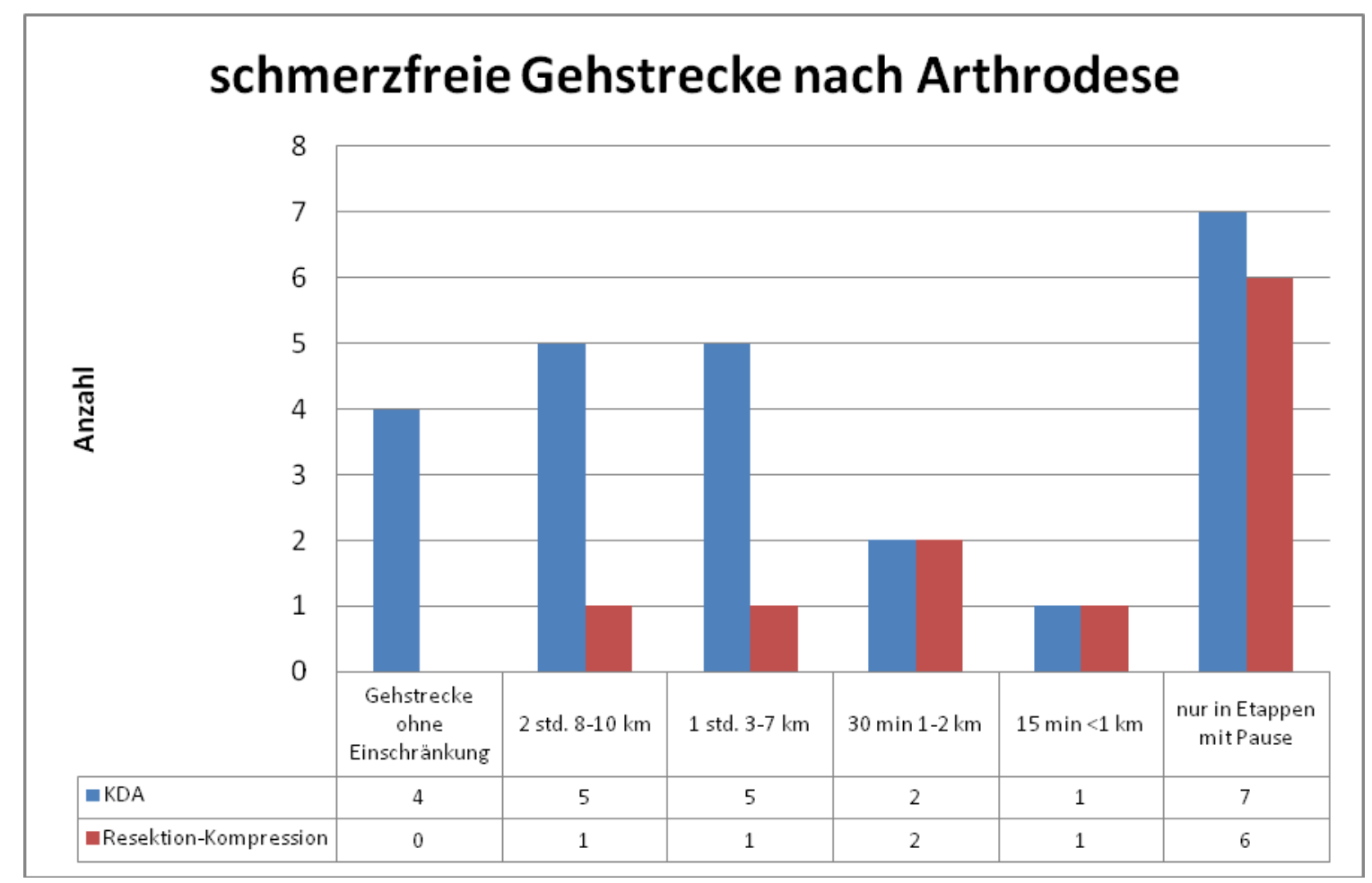

Abb.20: schmerzfreie Gehstrecke

\subsubsection{Subjektive Zufriedenheit}

KDA-Kollektiv

3 Patienten sind mit ihrem jetzigen Zustand subjektiv nicht zufrieden. Als kaum zufrieden bezeichnen sich 2 Personen mit ihrer Arthrodese. Eine leichte Zufriedenheit geben 7 Patienten $(29,17 \%)$ an. Sehr zufrieden sind mit dem Ergebnis 12 Patienten (50\%).

\section{Kompressions-Arthrodese-Kollektiv}

Hier gibt es keinen Patienten, der mit seinem Zustand überhaupt nicht zufrieden ist. Kaum zufrieden sind allerdings 3 Personen. Eine leichte Zufriedenheit beschreiben 4 Patienten $(36,26 \%)$. Mit ihrer Situation sind hier 4 Personen $(36,36 \%)$ sehr zufrieden.

Dies ist statistisch nicht signifikant. 


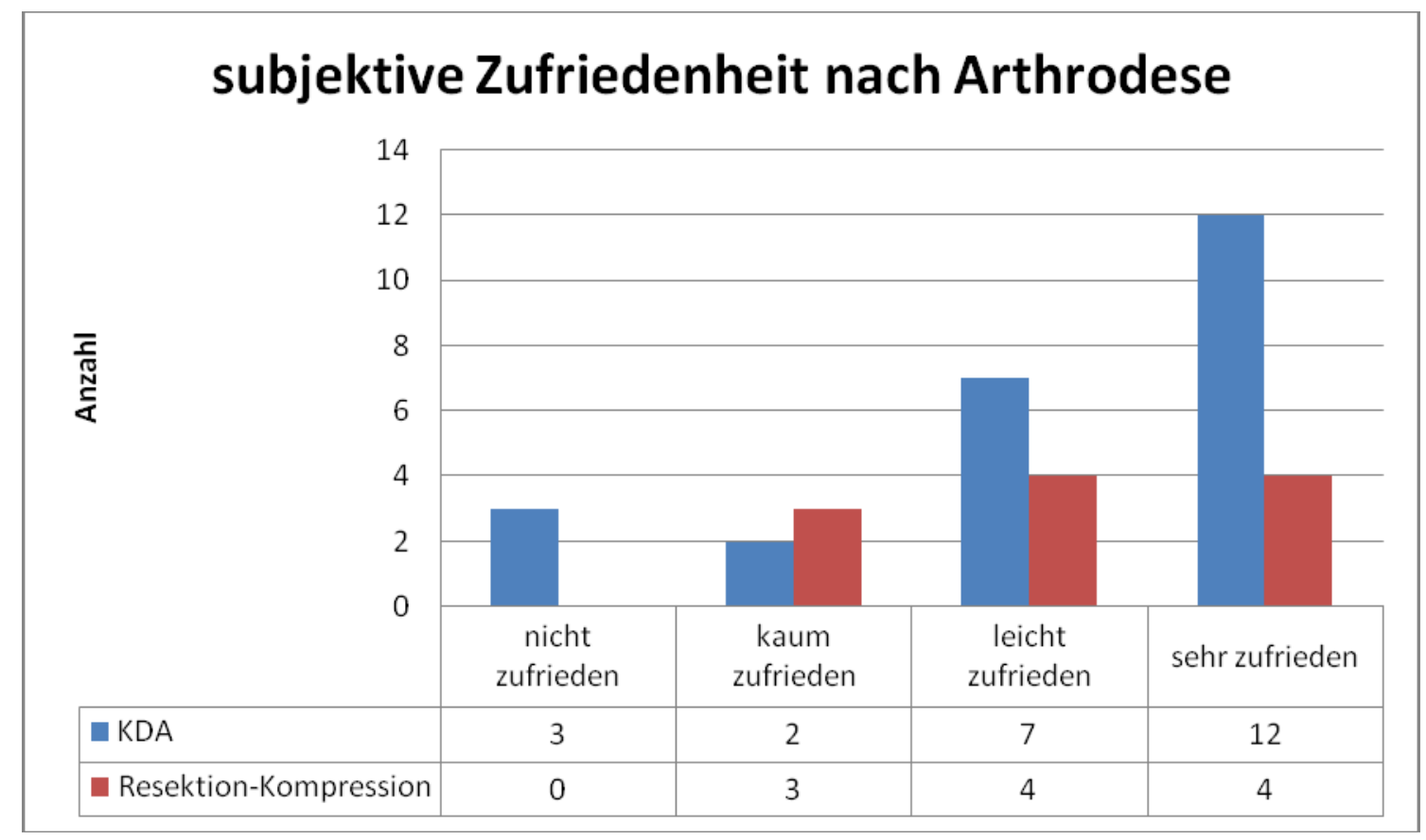

Abb.21: subjektive Zufriedenheit

\subsection{KDA am OSG versus KDA am USG}

Vergleich von Patienten mit einer KDA am OSG mit Patienten mit einer KDA am USG.

\subsubsection{Darstellung des KDA-Kollektives}

In dieser Gruppe befinden sich 24 Patienten. Von diesen wurde bei 13 (54,2\%) eine OSG- und bei 11 (45,8\%) eine USG-Versteifung durchgeführt. In der OSGGruppe befinden sich 9 (69,2\%) männliche und 4 (30,8\%) weibliche Patienten. Am USG bekamen 9 (81,8\%) Männer und 2 (18,2\%) Frauen eine KDA. 


\subsubsection{Altersverteilung}

\section{OSG-KDA}

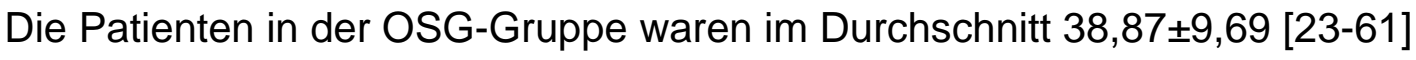
Jahre alt. Der Median lag hier bei 38,48 Jahren.

\section{USG-KDA}

In der USG-Gruppe waren die Patienten mit einem Altersdurchschnitt von 47,12 $\pm 11,13$ [20-58] Jahren und einem Median von 51,3 Jahren signifikant älter $(p=0,034)$.

\section{Altersverteilung der KDA-Patienten am USG}

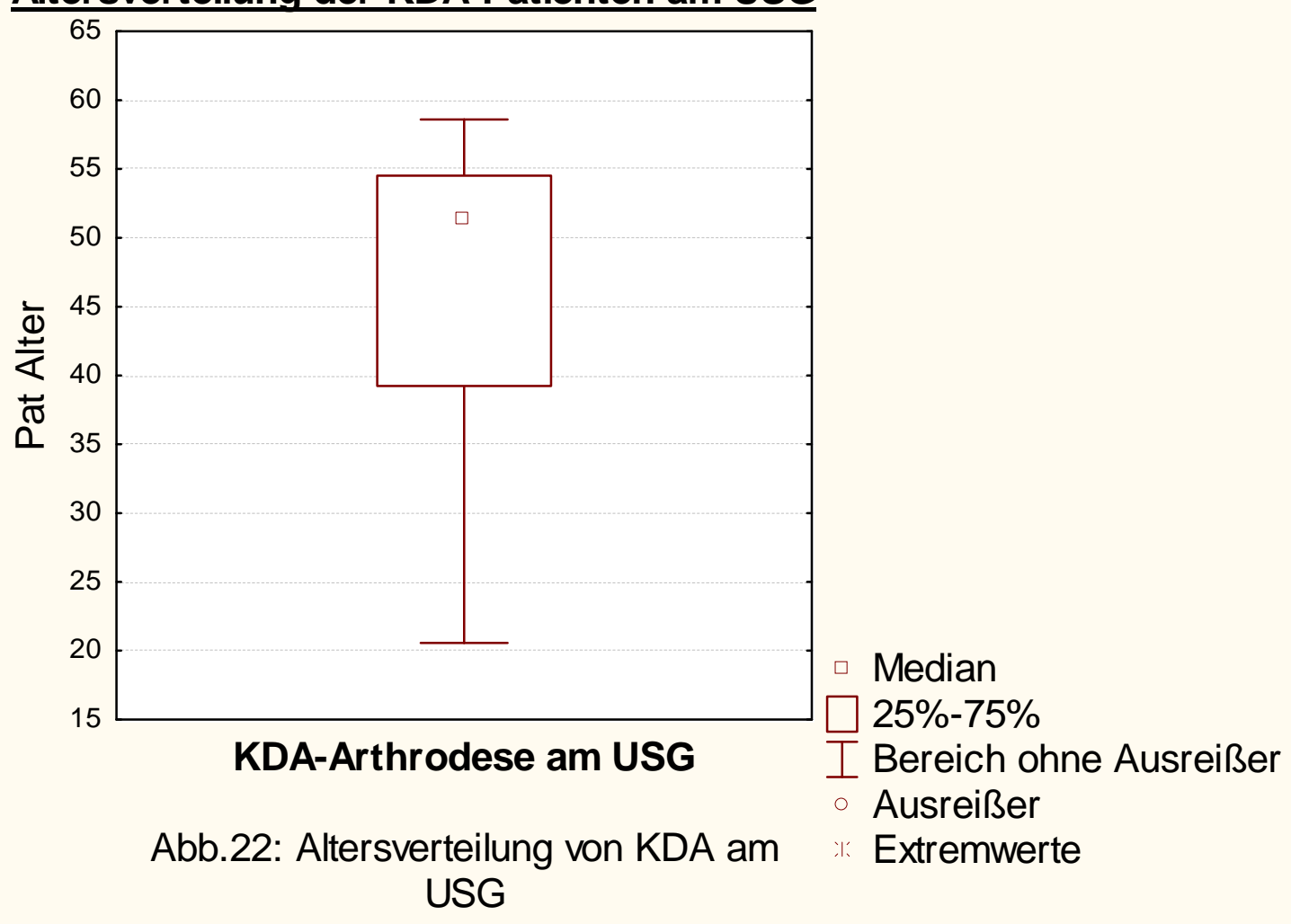




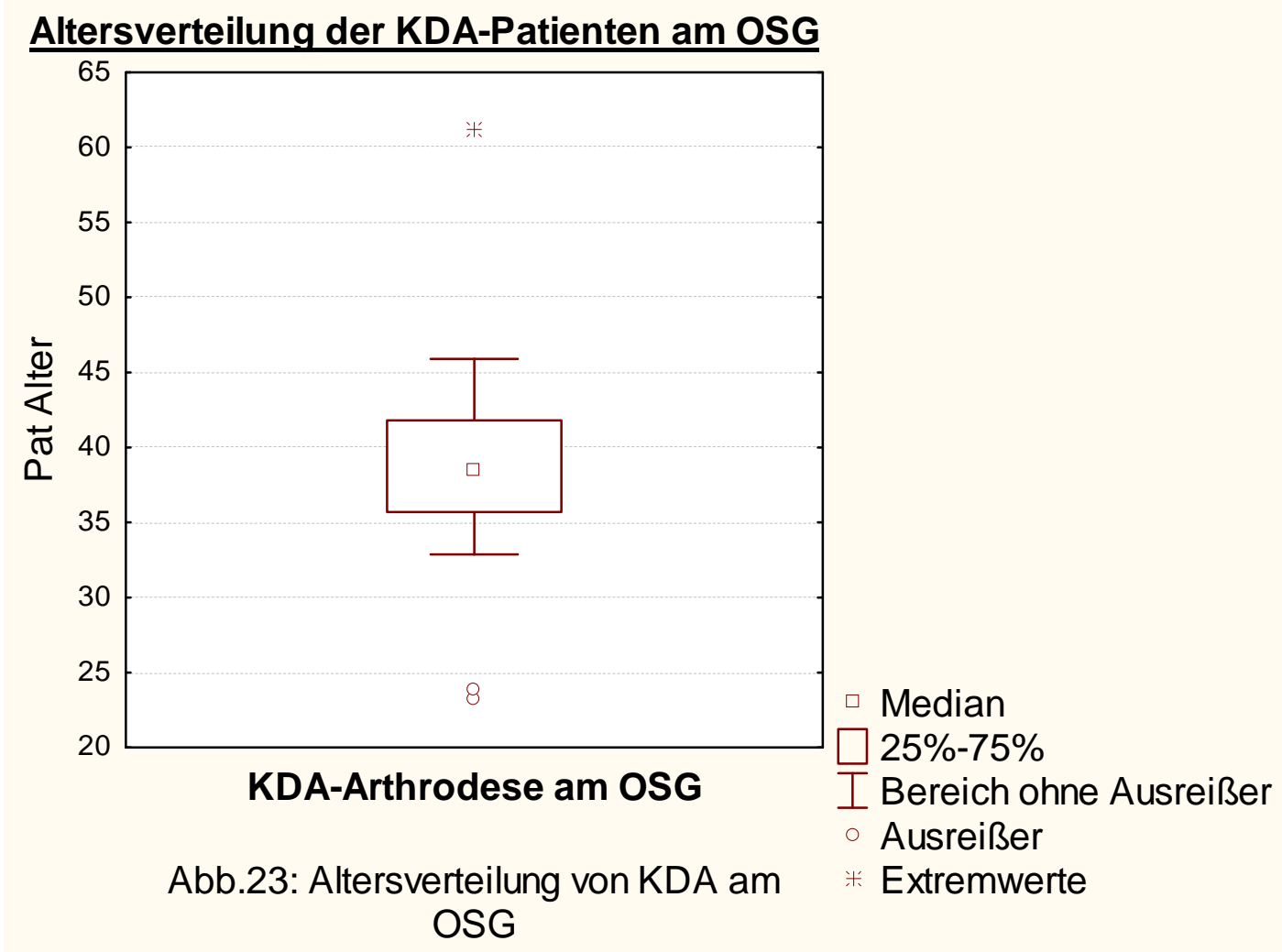

\subsubsection{Score-Ergebnisdarstellung}

\section{KDA-OSG-Kollektiv}

Die durchschnittlichen Score-Werte lagen bei dem KDA-OSG-Kollektiv beim Kitaoka-Score bei 76,3 $\pm 14,73$ [55-100] Punkten, beim McGuire-Score bei

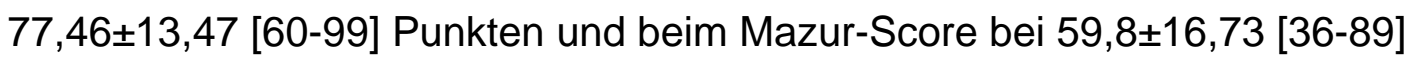
Punkten.

\section{KDA-USG-Kollektiv}

Bei dem KDA-USG-Kollektiv lagen die durchschnittlichen Score-Werte beim Kitaoka- Score bei 57,18 21,9 [29-90] Punkten, beim McGuire-Score bei

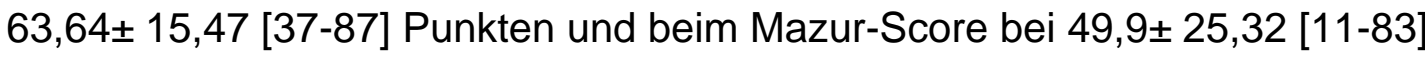
Punkten. 
Die Score-Ergebnisse fallen bei allen drei Scores in der OSG-Gruppe besser aus, als in der USG-Gruppe. Bei dem Kitaoka-Score ist dieser Unterschied auf dem Signifikanzniveau von Alpha=5\% signifikant $(p=0,043)$.

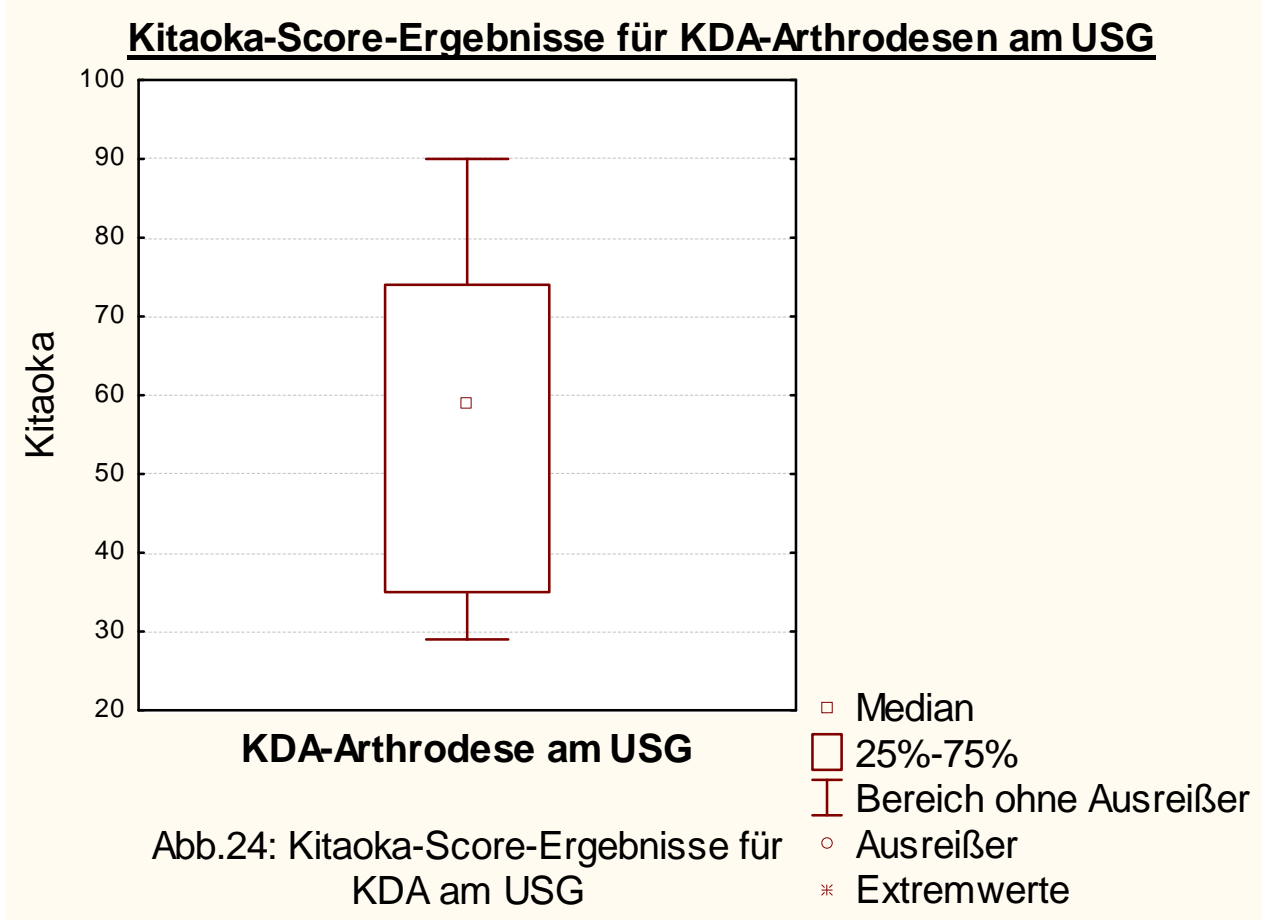

McGuire-Score-Ergebnisse für KDA-Arthrodesen am USG

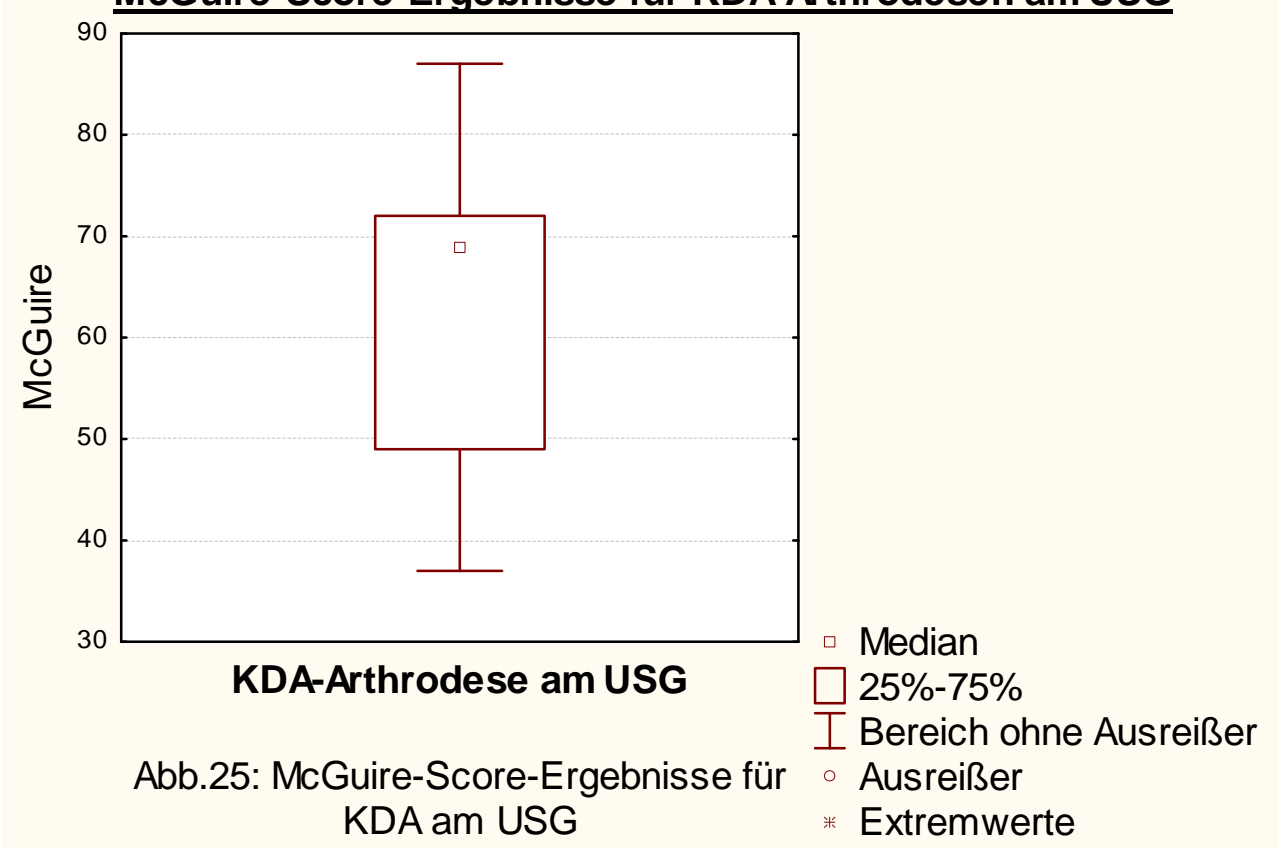



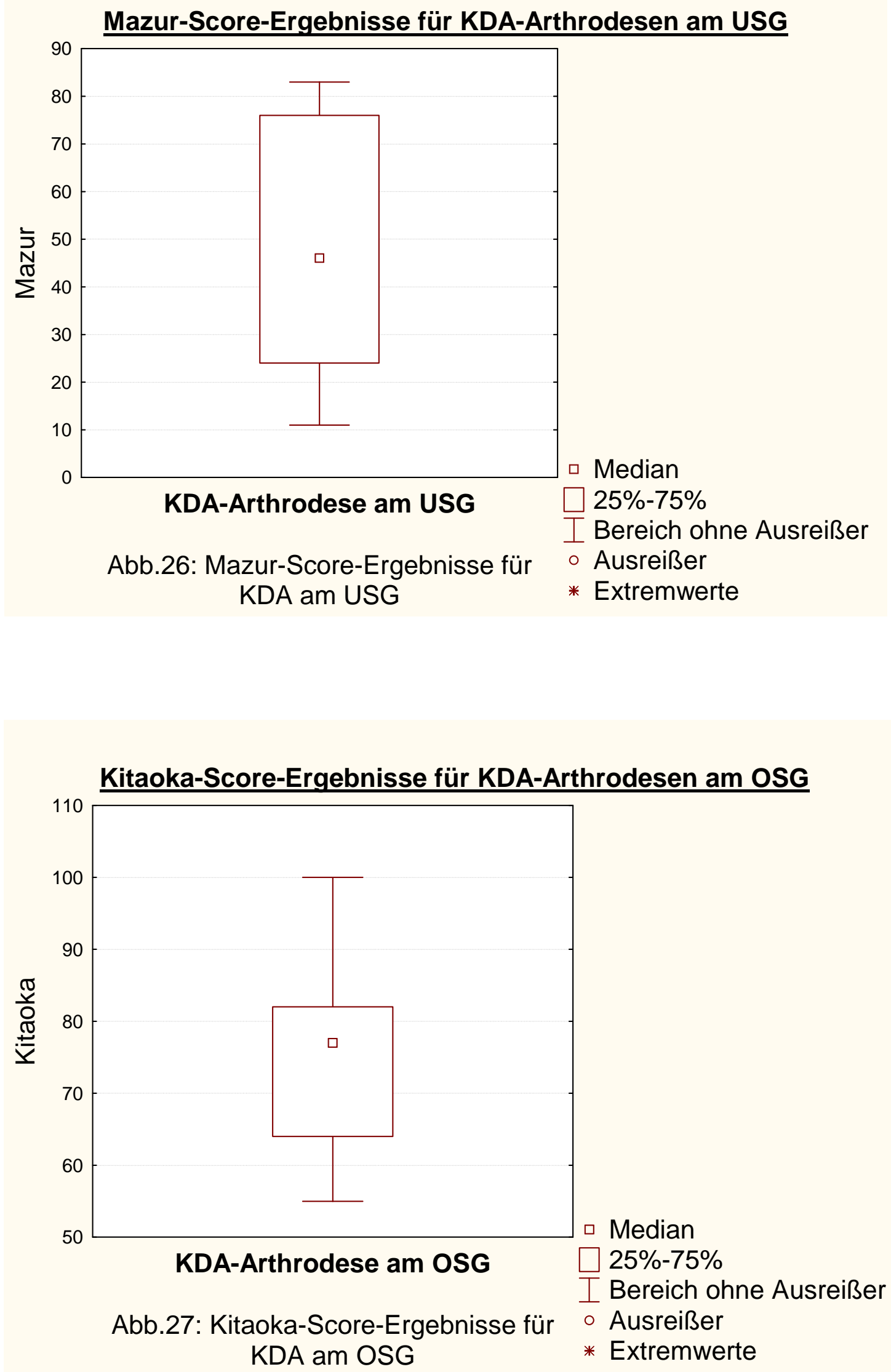

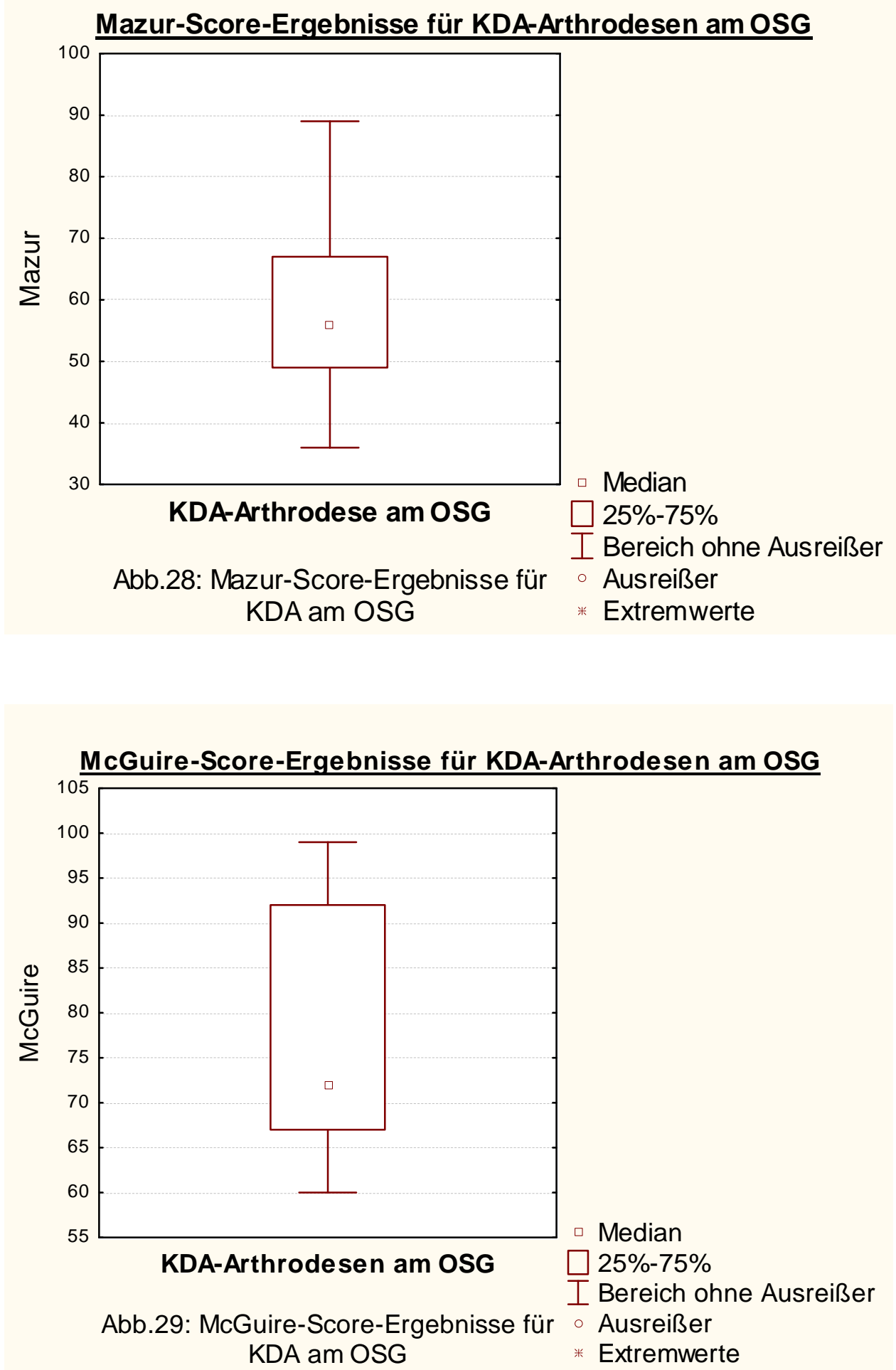


\subsubsection{Primäre Diagnosen}

\section{OSG-KDA}

In der Gruppe mit den am oberen Sprunggelenk durchgeführten KDAs hatten 5 Patienten (38,46\%) primär eine OSG-Fraktur. Weitere 8 Patienten $(61,54 \%)$ kamen ursprünglich mit einer Pilon-tibiale Fraktur.

\section{USG-KDA}

In dem Kollektiv mit der KDA am USG hatten 10 Patienten (90,91\%) initial eine Kalkaneusfraktur. Eine Person kam zu Beginn mit einer Talusfraktur. Diese Verteilung ist signifikant $(p=0,00003)$.

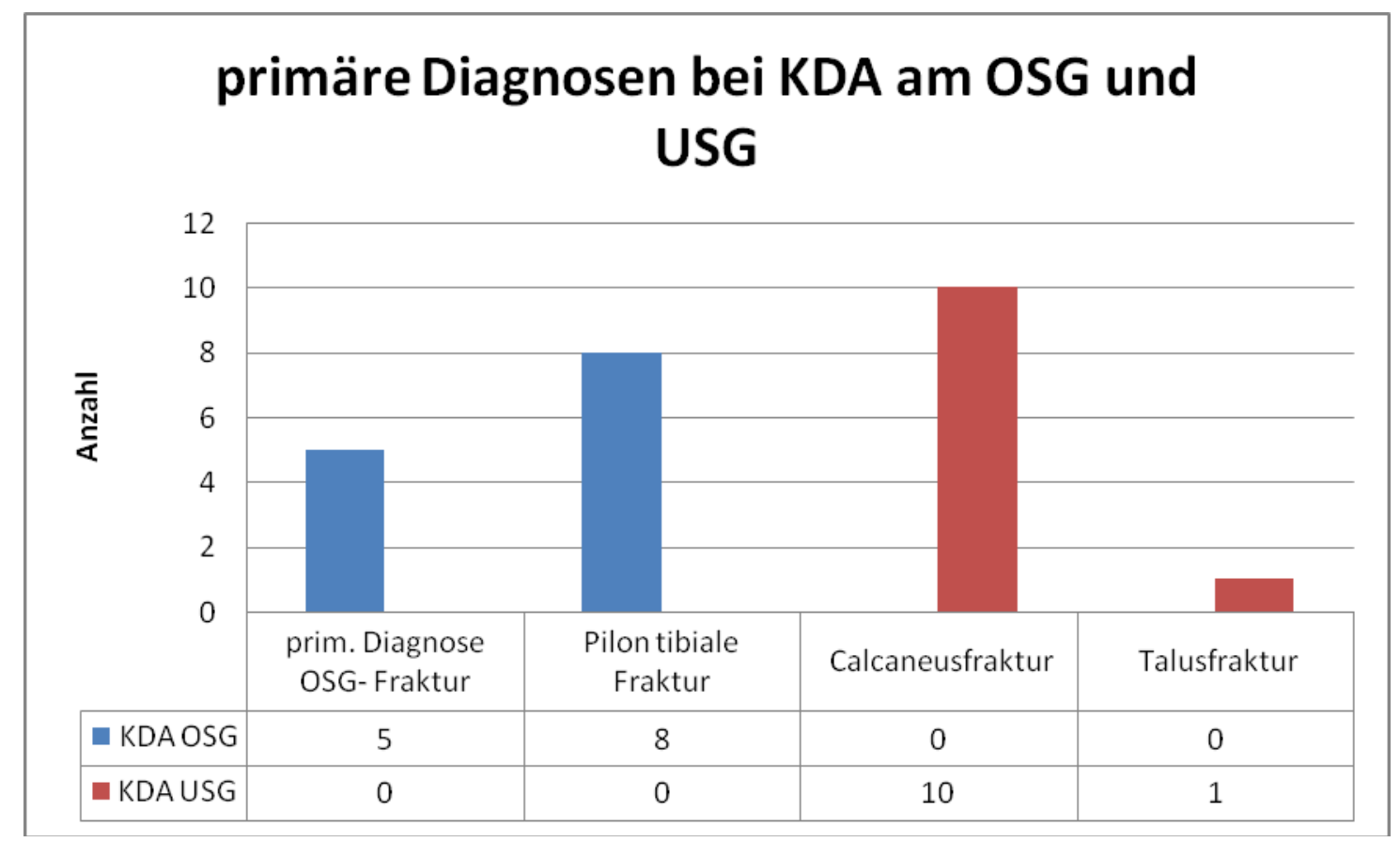

Abb.30: primäre Diagnosen 


\subsubsection{Operationsindikationen}

\section{OSG-KDA}

In der OSG-Gruppe wurde eine Operationsindikation aufgrund einer

Pseudarthrose gestellt. Eine exazerbierte Entzündung stellte ein weiteres Mal die Indikation zur OSG-Gelenkversteifung. Bei 11 Patienten (84,62\%) war ein persistierender, therapierefraktärer Schmerz Anlass zur KDA.

\section{USG-KDA}

In der USG-Gruppe war bei einem Patienten eine Pseudarthrose Grund zur Operation. Bei einer Person wurde aufgrund einer Talusnekrose die operative Versteifungsindikation gestellt. Hauptsächlich, nämlich bei 9 Patienten (81,82\%), führten persistierende Schmerzen zu dem Entschluss, eine KDA durchzuführen.

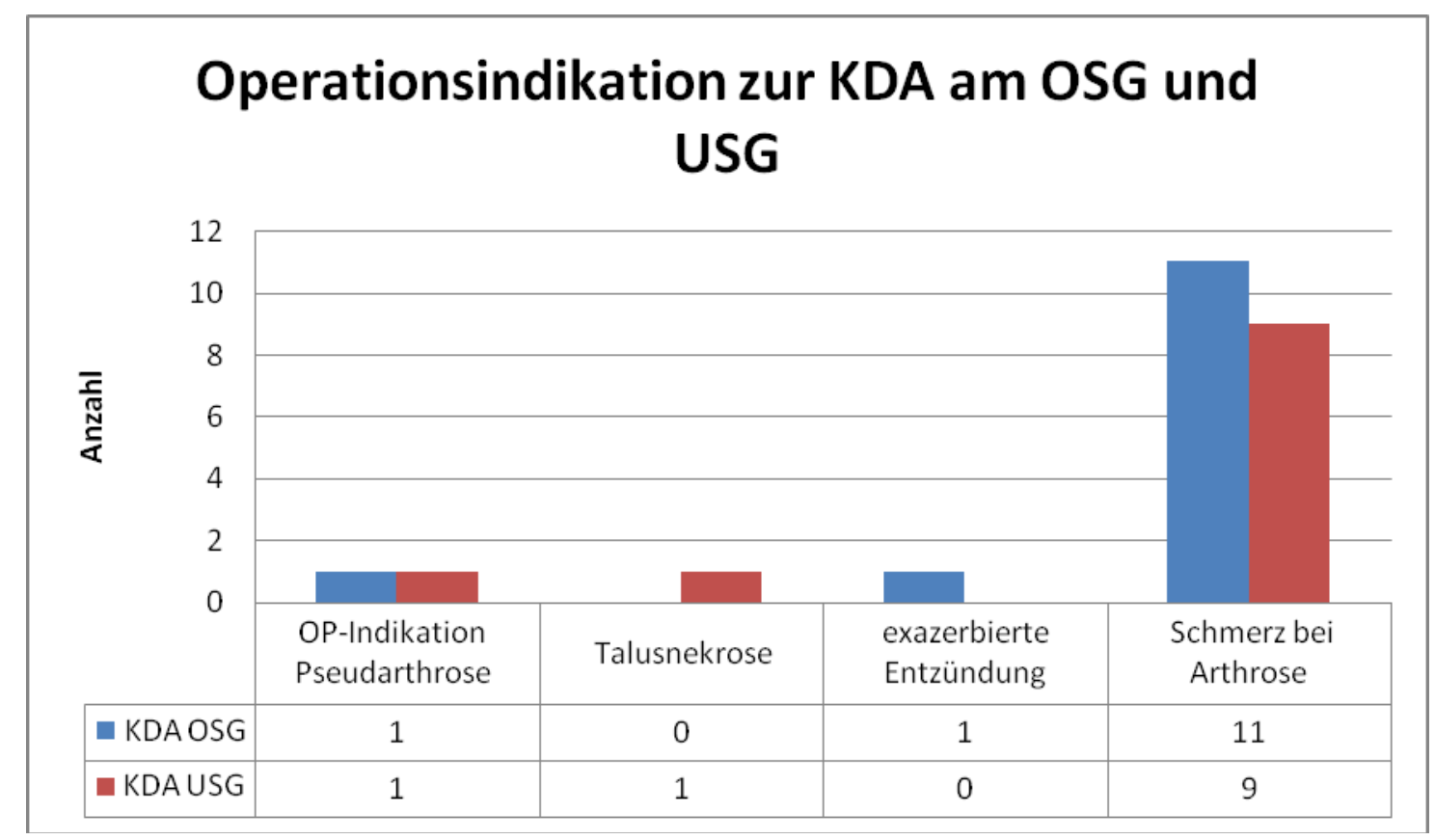

Abb.31: Indikationen zur KDA 


\subsubsection{Perioperative Komplikationen und Spätfolgen}

\section{OSG-KDA}

Bei 8 Patienten (61,54\%), die eine KDA am OSG bekommen haben traten keinerlei Probleme und Komplikationen auf. 2 Personen litten nach der OSGArthrodese unter einer Anschlussarthrose im USG. Bei einem Patienten trat zwei Jahre nach der Arthrodese ein Schraubenbruch auf. Eine Anschlussarthrose in den Tarsometatarsalgelenken manifestierte sich bei einer Person der OSG-Gruppe. In einem Fall trat ein lokaler postoperativer Infekt auf.

\section{$\underline{\text { USG-KDA }}$}

In der Gruppe der Patienten, die eine USG-Arthrodese bekamen war der postoperative Verlauf in 6 Fällen $(54,55 \%)$ unauffällig und komplikationslos. Bei 3 Patienten trat eine Anschlussarthrose im OSG auf. Eine Anschlussarthrose in den Tarsometatarsalgelenken wurde in einem Fall beobachtet. In dieser Gruppe der USG-Versteifungen trat ein Peronealsehnenimpingement zwei Jahre nach der Arthrodese- Operation auf. Signifikante Unterschiede konnten nicht beobachtet werden.

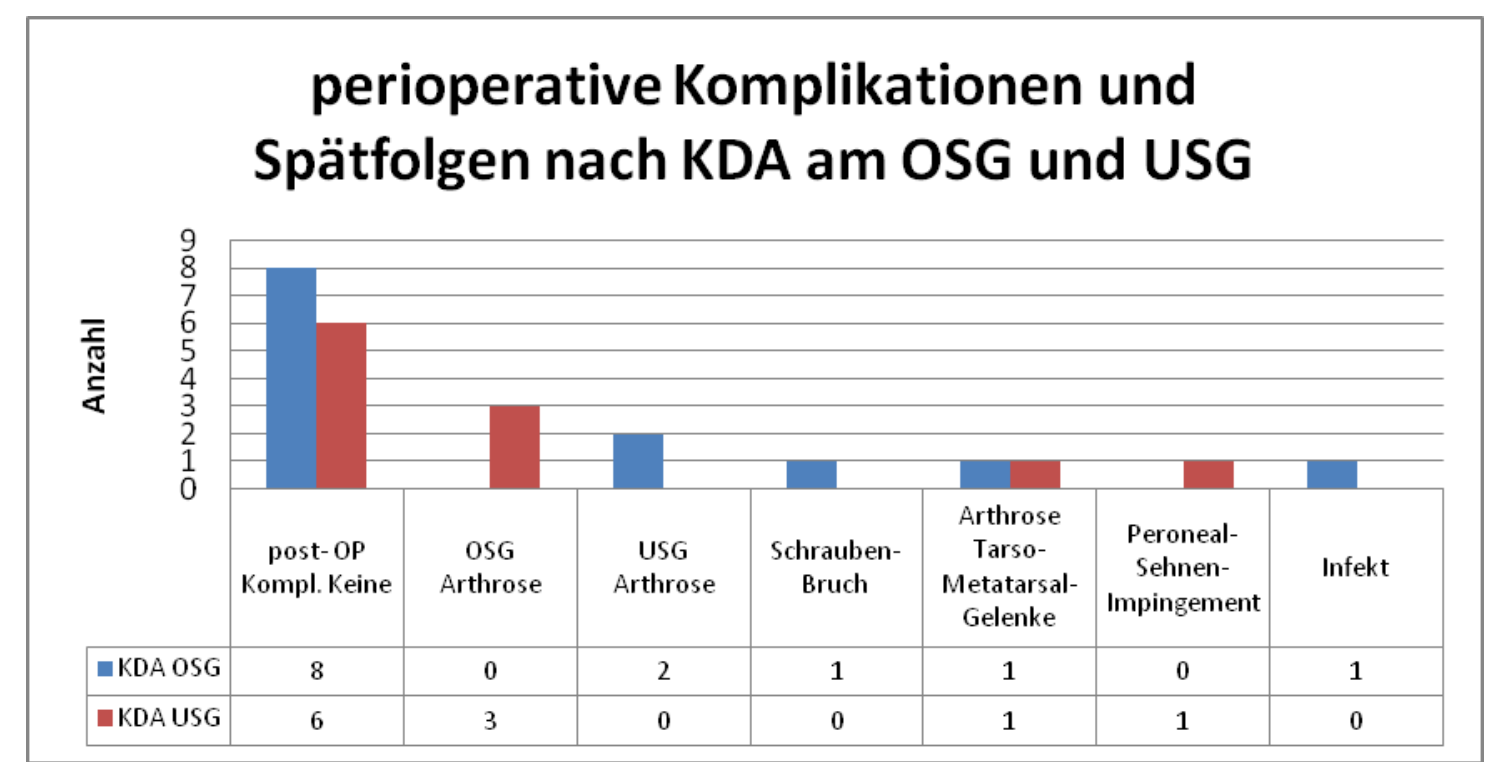

Abb.32: perioperative Komplikationen und Folgeprobleme 


\subsubsection{Schmerzen nach OP}

\section{OSG-KDA}

In dem Kollektiv der OSG-KDA-Patienten haben 3 Patienten keine Schmerzen. Über nur leichte Schmerzen berichten 4 Patienten $(30,77 \%)$ der OSG-Gruppe. Erträgliche Schmerzen haben 2 Personen, die im OSG versteift wurden. Ein Patient hat starke Schmerzen. 3 Personen berichten über Anlaufschmerzen.

\section{USG-KDA}

In der Gruppe der am USG versteiften Patienten gibt es keinen, der über eine absolute Schmerzfreiheit berichten kann. Bei den Patienten, bei denen eine KDA am USG gemacht wurde gibt es 4 Personen (36,36\%), die über noch leichte Schmerzen berichten. Erträgliche Schmerzen haben hier 3 Patienten. 3 Personen aus der USG-Gruppe berichten über starke Schmerzen.

Ein Patient klagt über Anlaufschmerzen. Eine statistische Signifikanz ließ sich nicht nachweisen.

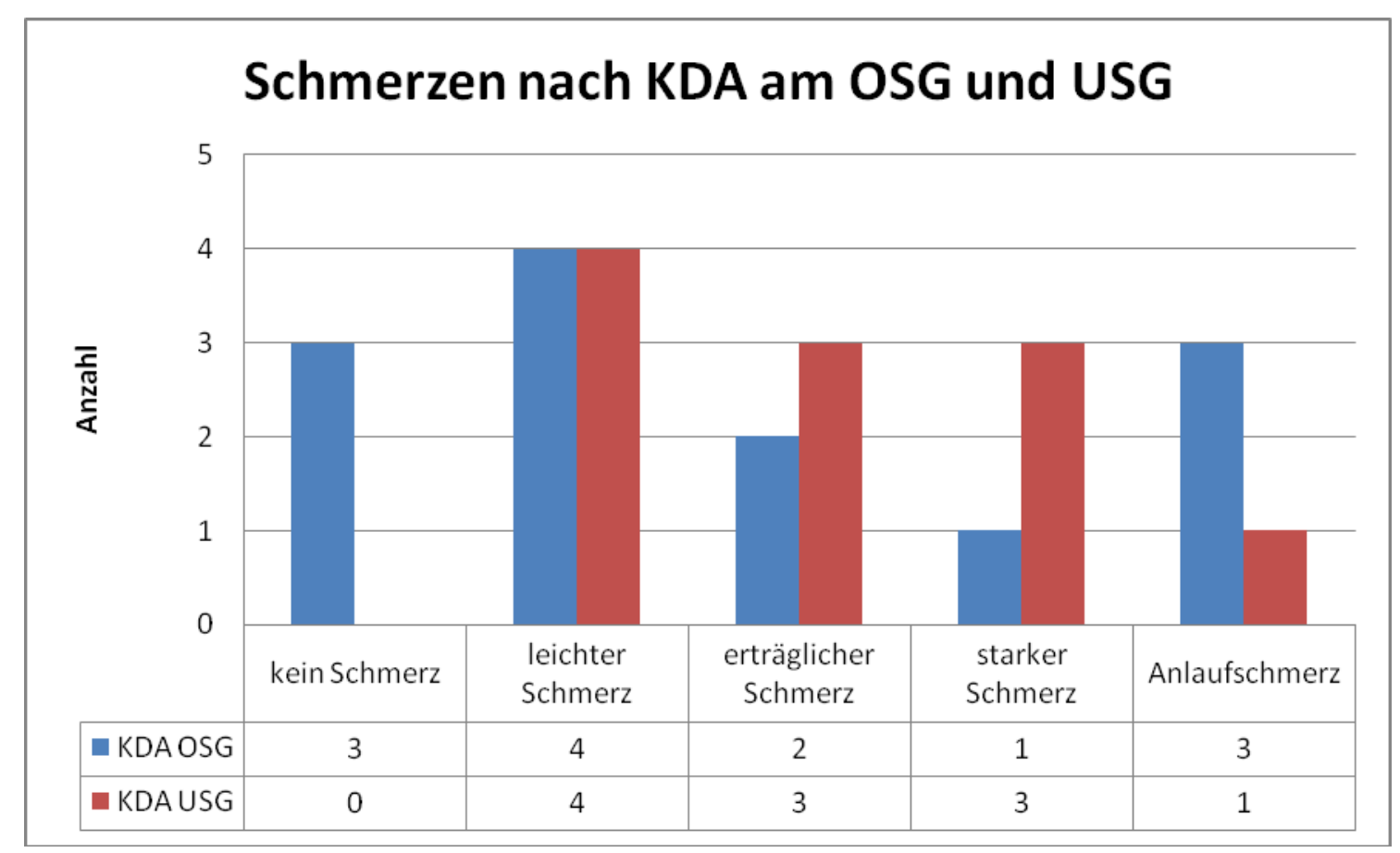

Abb.33: Schmerzen 


\subsubsection{Belastungsabhängige Schmerzen nach OP}

\section{OSG-KDA}

3 Personen haben aus dem OSG-Kollektiv nie Schmerzen unter Belastung. Über gelegentliche Schmerzen unter Belastung berichten 5 Patienten $(38,46 \%)$. 3 Personen haben bei Belastungen kontinuierliche Schmerzen. Ruhe- oder Spontanschmerzen haben 2 Personen

\section{USG-KDA}

Bei der Betrachtung des USG-Kollektives ist hier ein Patient, der nie Schmerzen unter Belastung hat. Weiter gibt es in diesem Kollektiv 5 Patienten $(45,45 \%)$, die gelegentlich Schmerzen unter Belastung haben. Eine Person berichtet über kontinuierliche Belastungsschmerzen. Über Ruhe- oder Spontanschmerzen berichten 4 Personen $(36,36 \%)$.

Signifikante Ergebnisse sind nicht zu ermitteln.

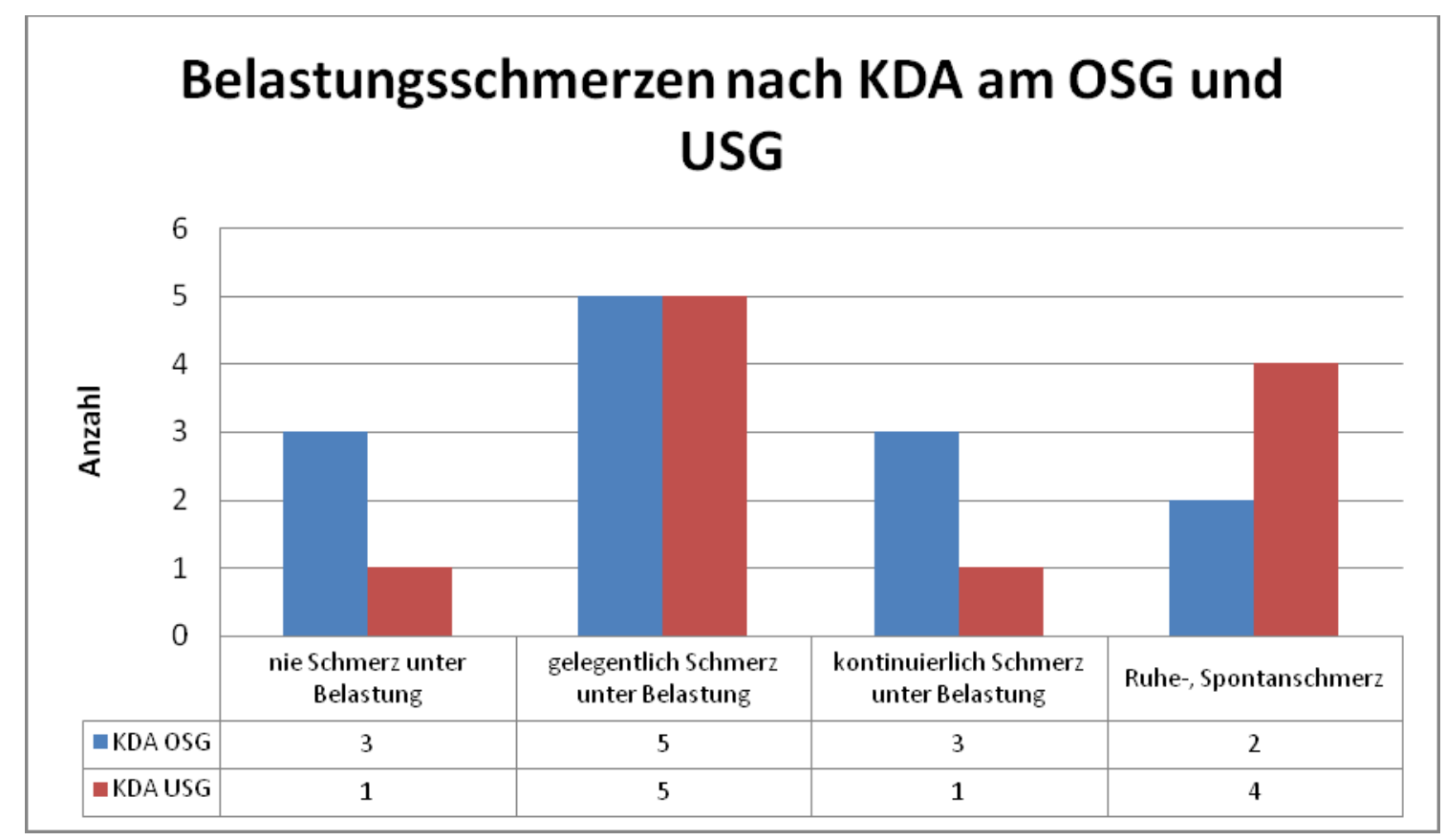

Abb.34: Belastungsschmerzen 


\subsubsection{Dauerhafte Schmerzmitteleinnahme nach OP}

\section{OSG-KDA}

8 Patienten $(61,54 \%)$ nehmen nie Schmerzmittel ein. 1-2 Mal pro Woche nehmen 4 Personen $(30,77 \%)$ Schmerzmittel ein. Eine Person nimmt in diesem Kollektiv täglich Schmerzmittel.

\section{USG-KDA}

In dieser Gruppe nehmen 6 Personen (54,55\%) nie Schmerzmittel ein. Ein Patient nimmt 1-2 Mal pro Woche Schmerzmittel ein. 2 Personen geben an 3-5 Mal pro Woche Schmerzmittel einzunehmen. Weitere 2 Patienten nehmen täglich Schmerzmittel.

Bei dem Vergleich der dauerhaften Schmerzmitteleinnahme bei Patienten mit KDA am OSG oder am USG sind statistisch keine Signifikanzen oder hinweisende Unterschiede zu beobachten.

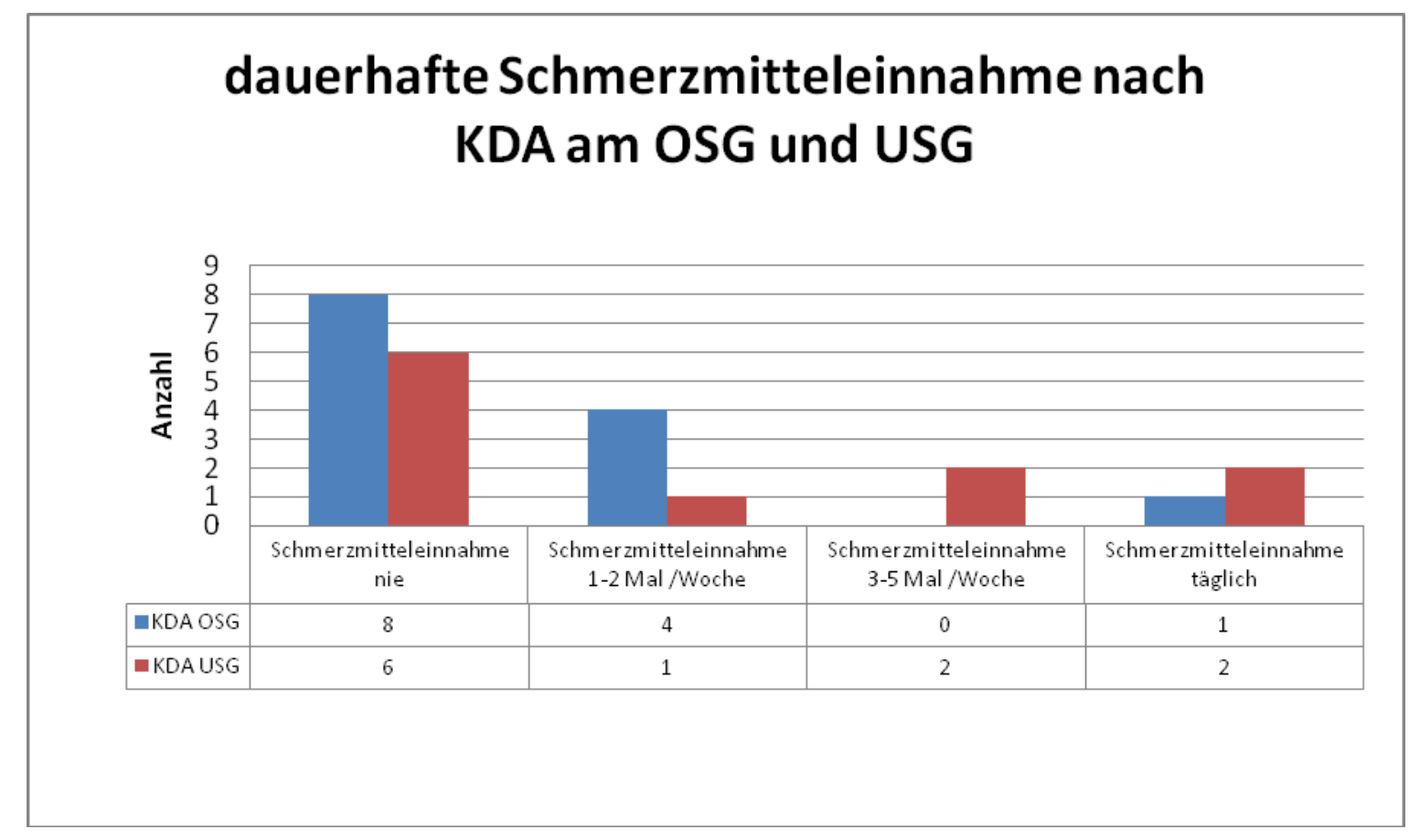

Abb.35: dauerhafte Schmerzmitteleinnahme 


\subsubsection{Schmerzfreie Gehstrecke nach OP}

OSG-KDA

In der OSG-Gruppe können 3 Patienten ohne Einschränkungen gehen. Weitere 4 Personen $(30,77 \%)$ geben an maximal 2 Stunden schmerzfrei gehen zu können. In 2 Fällen ist ein schmerzfreies Gehen über 1 Stunde möglich. 30 Minuten Gehen ohne Schmerzen zu haben, können in diesem OSG-Kollektiv 2 Personen. Ebenfalls 2 Patienten berichten, dass ein schmerzfreies Gehen nur in Etappen mit Pausen möglich ist.

\section{USG-KDA}

In der Gruppe mit der KDA am USG kann im Vergleich einer ohne Einschränkungen schmerzfrei Gehen. Ein Patient berichtet hier über die Möglichkeit über eine Dauer von 2 Stunden schmerzfrei unterwegs sein zu können. In 3 Fällen ist ein schmerzfreies Gehen über eine Stunde möglich. Eine schmerzfreie Gehstrecke über 15 Minuten ist in einem Fall möglich. In dieser Gruppe sind es 5 Personen (45,45\%), die eine schmerzfreie Gehstrecke nur in Etappen mit Pausen erreichen.

Statistische Signifikanzen lassen sich nicht nachweisen. 


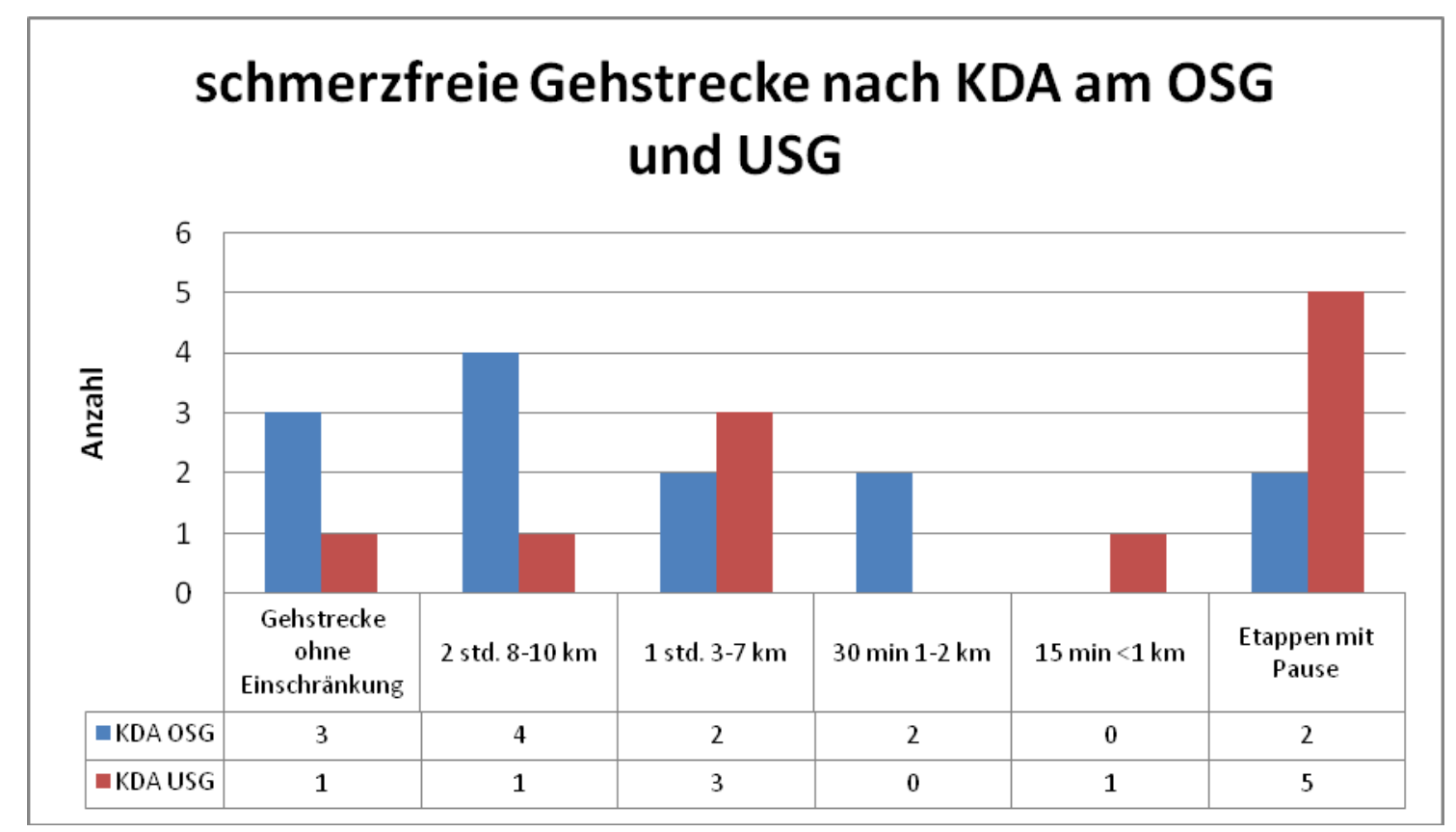

Abb.36: schmerzfreie Gehstrecke

\subsubsection{Subjektive Zufriedenheit}

OSG-KDA

In der OSG-Gruppe gibt es keinen, der nicht zufrieden oder kaum zufrieden ist.

4 Patienten $(30,77 \%)$ sind in dieser Gruppe leicht zufrieden. Sehr zufrieden sind 9 Patienten $(69,23 \%)$.

\section{USG-KDA}

In der Gruppe der KDA des USG sind 3 Patienten unzufrieden. Weitere 2 Personen geben an kaum zufrieden zu sein. 3 Patienten sind leicht zufrieden. In dieser Gruppe sind 3 Personen sehr zufrieden.

Die Patienten, die eine KDA am OSG bekommen haben sind statistisch signifikant zufriedener, als die Patienten mit einer KDA am USG $(p=0,045)$. 


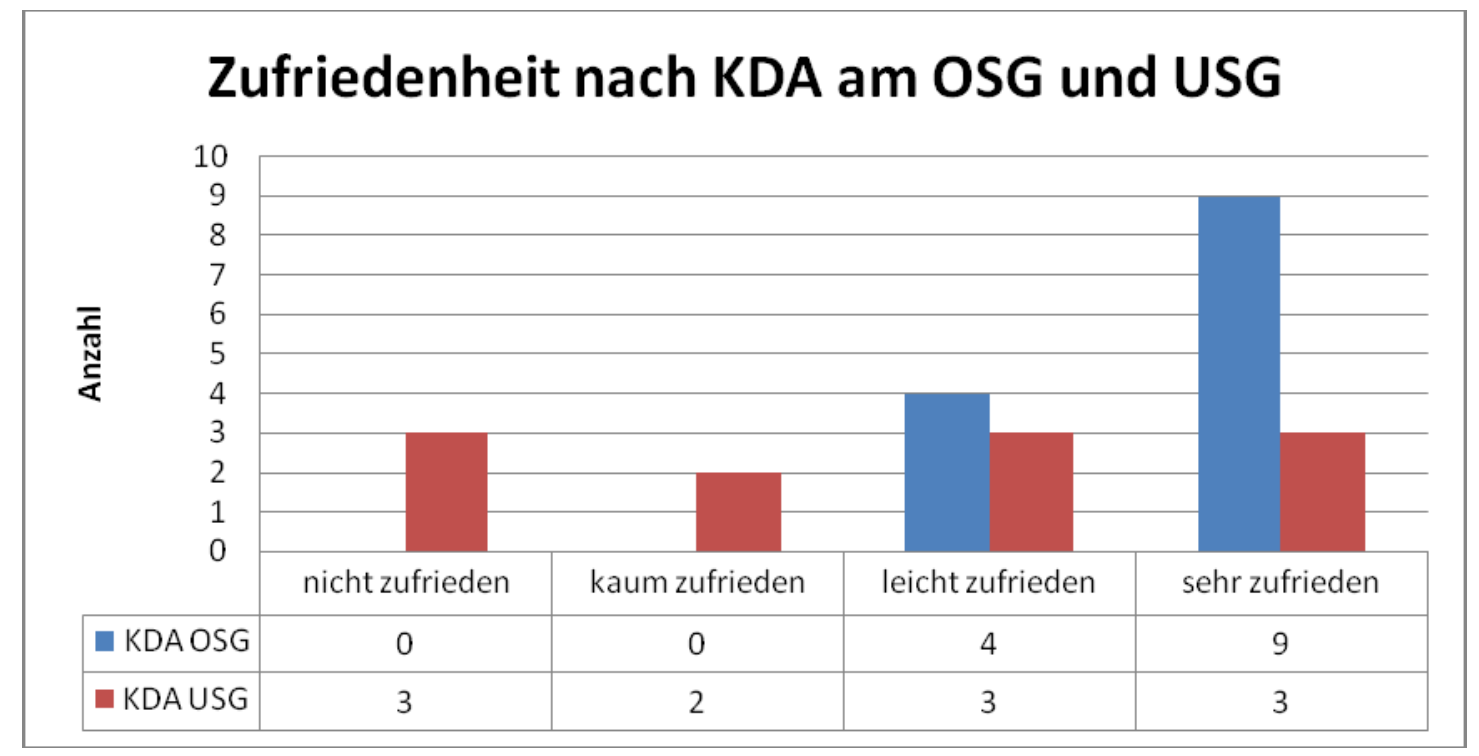

Abb.37: Zufriedenheit

\subsection{KDA am OSG versus Kompressionsarthrodese am OSG}

Vergleich von Patienten mit einer KDA am OSG mit Patienten mit einer Kompressionsarthrodese am OSG.

\subsubsection{Darstellung des OSG-Kollektives}

Von den insgesamt 21 durchgeführten OSG-Arthrodesen wurden 13 (61,9\%) mit der KDA und $8(38,1 \%)$ mit dem Kompressionsverfahren operiert. In der KDA-Gruppe waren 9 (69,2\%) männliche und 4 (30,8\%) weibliche Patienten. Die Kompressionsarthrodese wurde bei 5 männlichen $(62,5 \%)$ und 3 weiblichen Personen durchgeführt. 


\subsubsection{Altersverteilung}

\section{$\underline{\text { KDA-OSG }}$}

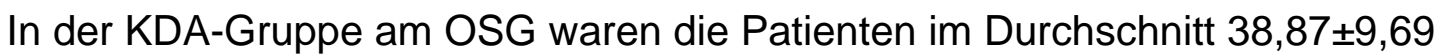
[23-61] Jahre alt. Der Median lag hier bei 38,48 Jahren.

\section{Kompressions-Arthrodese-OSG}

Der Altersdurchschnitt lag in der Kompressions-Gruppe bei 47,16 $\pm 18,95$ [20-74] Jahren. Der Median lag bei 46,9 Jahren. Somit waren die Patienten aus der OSG-KDA-Gruppe etwas jünger als diejenigen aus der OSG-KompressionsGruppe. Eine Signifikanz ergibt sich jedoch nicht.

\section{Altersverteilung der OSG versteiften Patienten durch KDA bzw. Kompressions-} Arthrodese

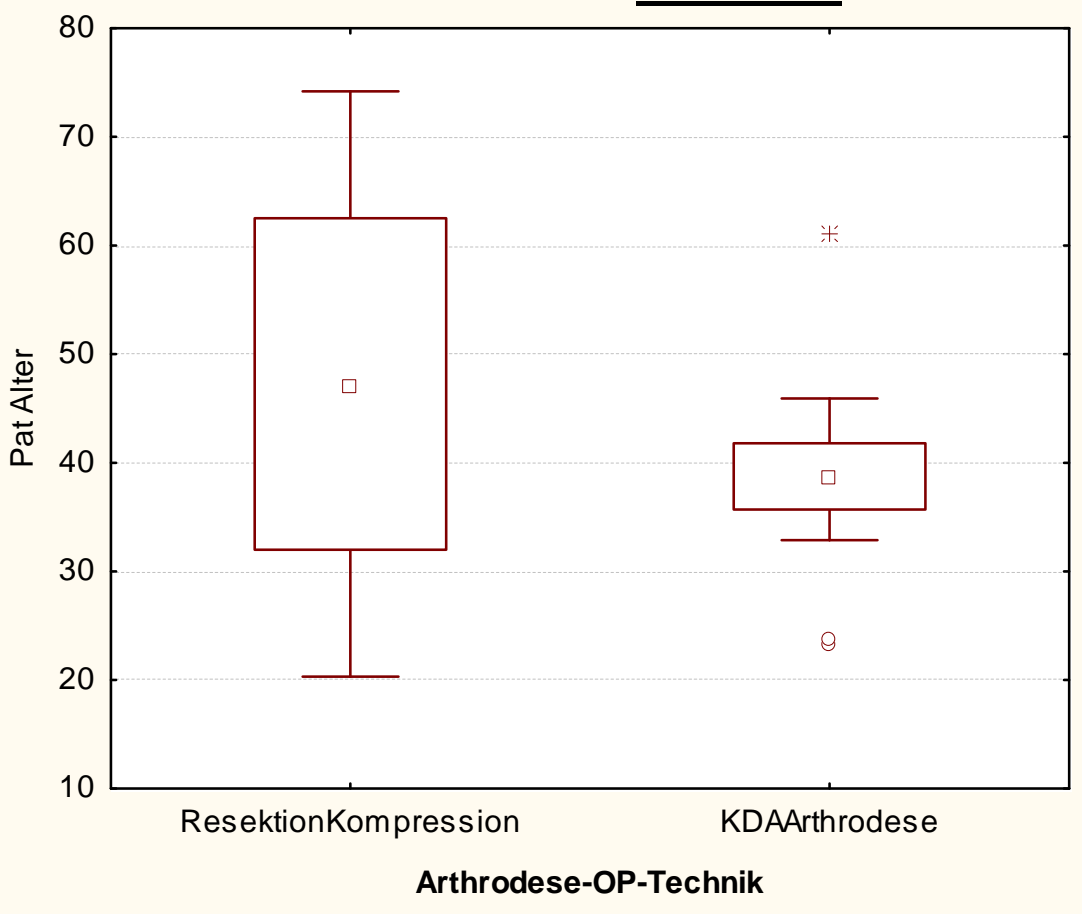

Abb.38: Altersverteilungen bei KDA und Kompressionsarthrodese am OSG
Median

25\%-75\%

I Bereich ohne Ausreißer Ausreißer

* Extremwerte 


\subsubsection{Score-Ergebnisdarstellung}

\section{KDA-OSG}

Bei der KDA am OSG kam der Kitaoka-Score auf durchschnittliche 76,3 $\pm 14,7$ [55-100] Punkte, der Mazur-Score auf 59,8 816,7 [36-89] Punkte und der McGuire-Score auf durchschnittliche 77,5 $\pm 13,5$ [60-99] Punkte.

\section{Kompressions-Arthrodese-OSG}

Bei der Kompressionsarthrodese am OSG ergab der Kitaoka-Score

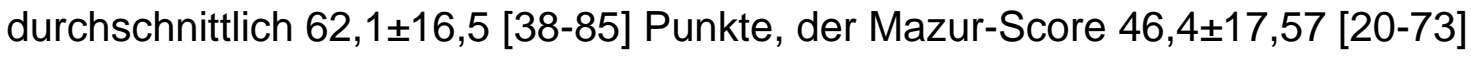
Punkte und der McGuire-Score 64,1£11,2 [48-82] Punkte.

Die erreichten Punkte bei allen drei Scores liegen in der Gruppe von Patienten, die mit der KDA am OSG versteift wurden höher, als in der Gruppe mit den OSG-Arthrodesen durch die Resektions-Kompressionsarthrodese. Bei dem McGuire-Score sind die Ergebnisse im Vergleich beider Gruppen signifikant $(p=0,025)$.

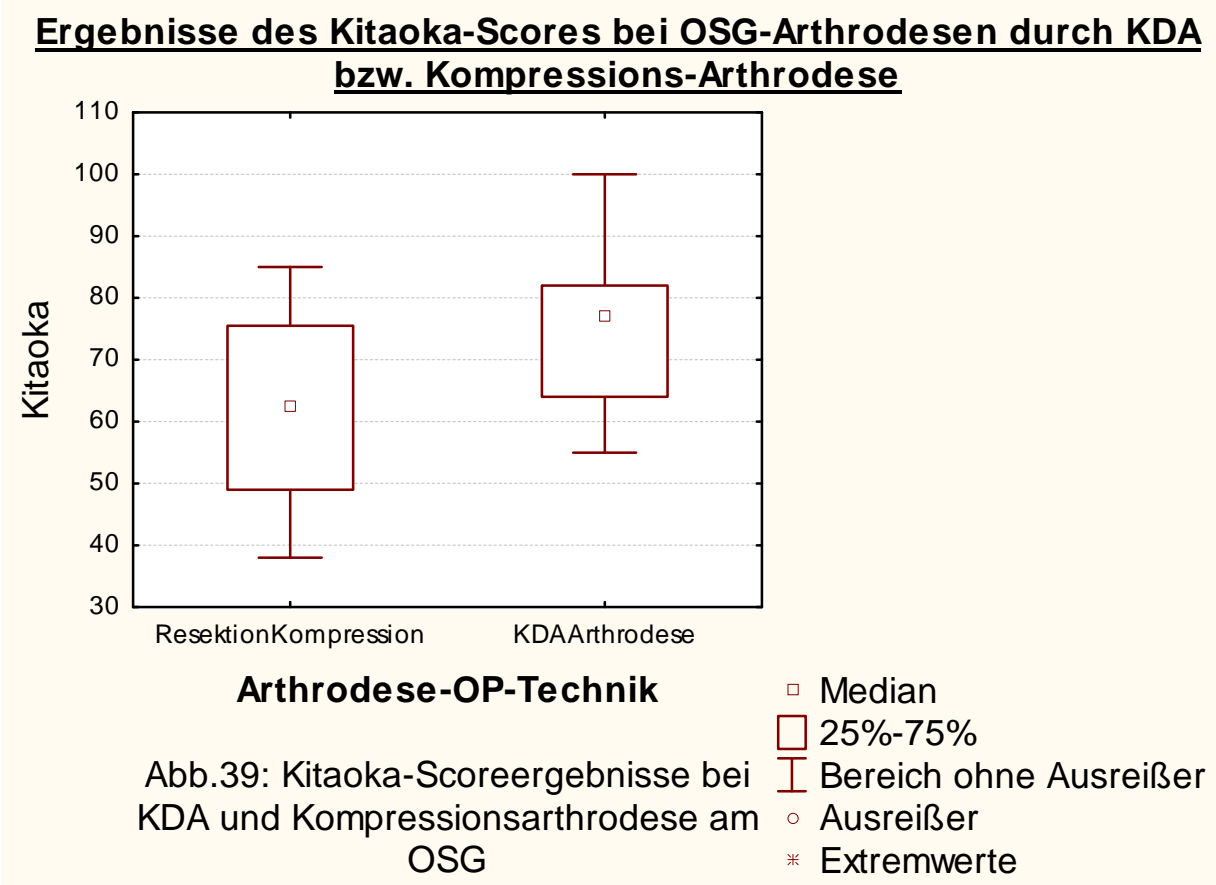


Ergebnisse des Mazur-Scores bei OSG-Arthrodesen durch KDA bzw.

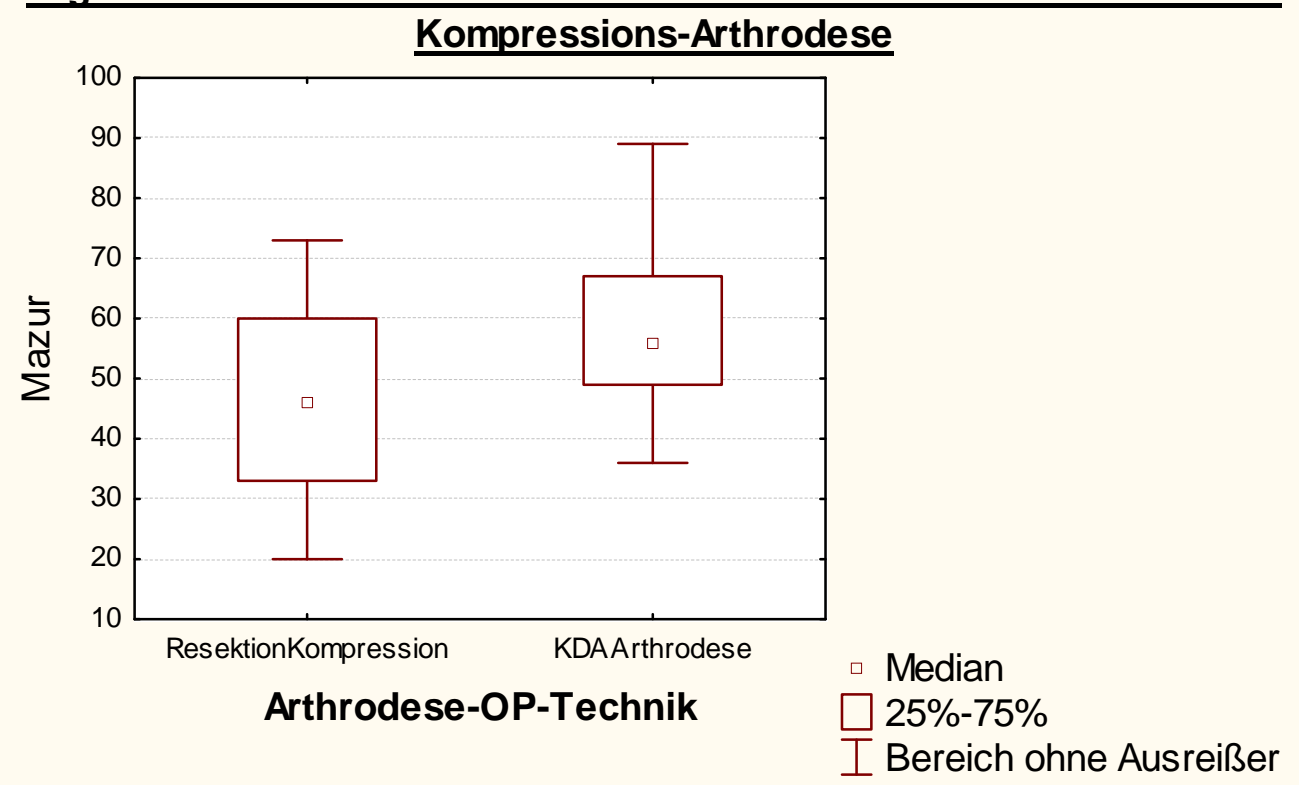

Abb.40: Mazur-Scoreergebnisse bei KDA Ausreißer und Kompressionsarthrodese am OSG * Extremwerte

Ergebnisse des McGuire-Scores bei OSG-Arthrodesen durch KDA bzw. Kompressions-Arthrodese

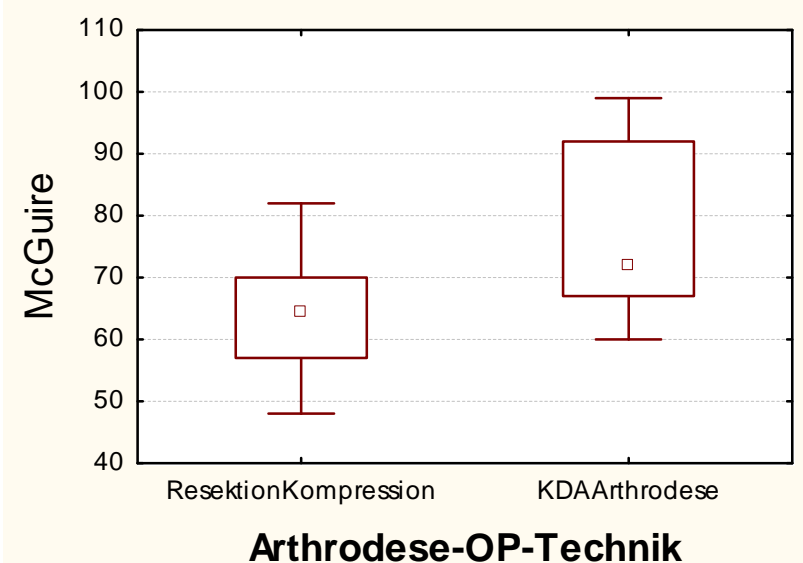

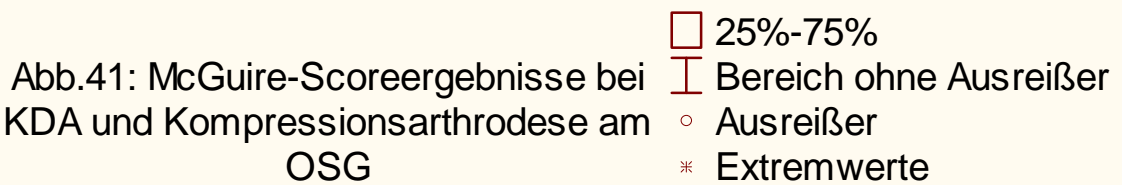




\subsubsection{Primäre Diagnosen}

\section{$\underline{\text { KDA-OSG }}$}

In der Gruppe der KDA am OSG waren die primären Diagnosen 5 OSG-

Frakturen $(38,46 \%)$ und 8 Pilon-tibiale Frakturen $(61,54 \%)$.

\section{Kompressions-Arthrodese-OSG}

In dem Kollektiv der Kompressions-Arthrodesen am OSG gab es 5 Pilon-tibiale Frakturen (62,5\%), eine Talusfraktur, eine traumatische Fraktur einer vorbestehenden Arthrodese und eine kombinierte Pilon- und Kalkaneusfraktur.

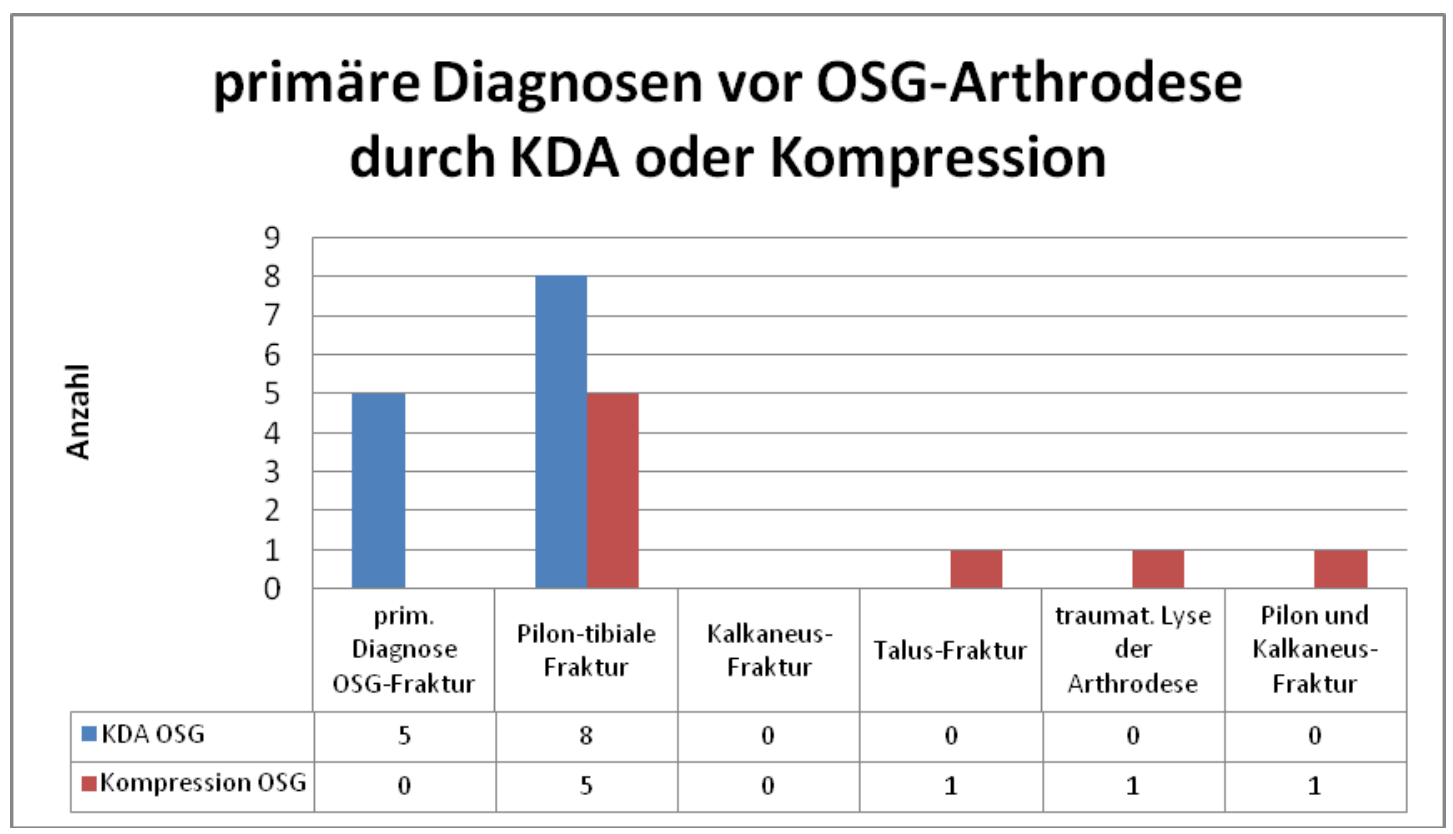

Abb.42: primäre Diagnosen vor OSG-Arthrodese 


\subsubsection{Operationsindiktionen}

\section{KDA-OSG}

Zu einer KDA am OSG führten eine Pseudarthrose, eine exazerbierte Entzündung und 11 therapierefraktäre Schmerzsymptomatiken (84,62\%).

\section{Kompressions-Arthrodese-OSG}

In der Gruppe der Kompressionsarthrodesen am OSG hatten drei Patienten primär eine Pseudarthrose. Bei einer Person war eine exazerbierte Entzündung Indikation zur Versteifungsoperation. Bei weiteren 4 Patienten (50\%) wurde die OSG-Kompressions-Arthrodese wegen einer persistierenden Schmerzsymptomatik vorgenommen. Hier war in beiden Gruppen vor allem ein persistierender, meist posttraumatischer Schmerz die Indikation zur Arthrodese.

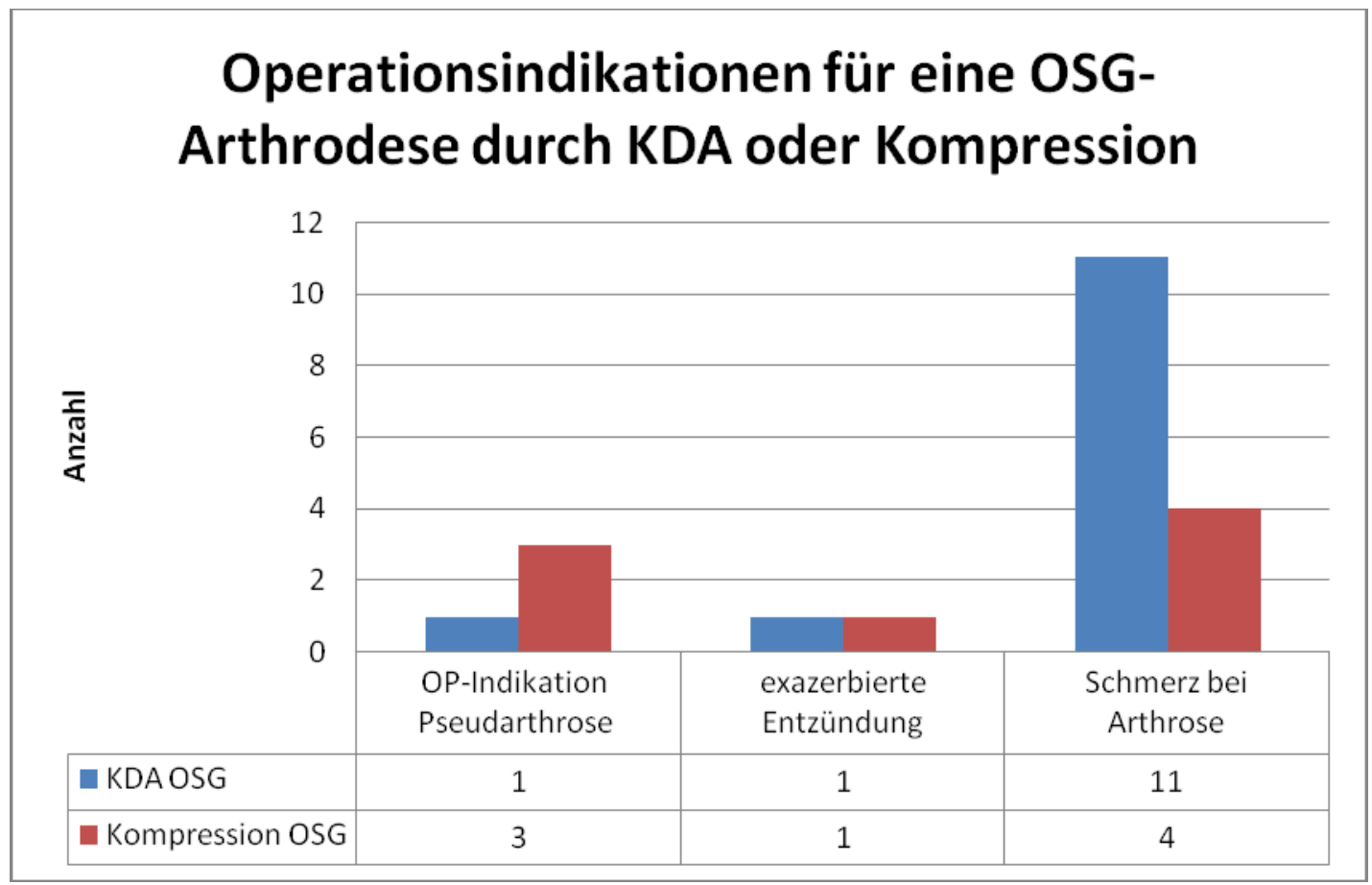

Abb.43: Operationsindiaktionen für eine OSG-Arthrodese 


\subsubsection{Perioperative Komplikationen und Spätfolgen}

\section{KDA-OSG}

Bei den Patienten mit einer OSG-Arthrodese durch KDA hatten 8 Personen $(61,54 \%)$ keinerlei Komplikationen oder Spätfolgen. Eine Anschlussarthrose im USG entwickelten zwei Patienten. In einem Fall trat ein Schraubenbruch nach zwei Jahren auf. An einer Anschlussarthrose in den Tarsometatarsalgelenken litt eine Person im späteren Verlauf. Postoperativ manifestierte sich bei einem Patienten ein lokaler Infekt.

\section{Kompressions-Arthrodese-OSG}

In dem Kollektiv der OSG versteiften Patienten durch die KompressionsArthrodese waren 4 Patienten (50\%) ohne Komplikationen bzw. Spätfolgen. In einem Fall wurde eine Anschlussarthrose in den Tarsometatarsalgelenken beobachtet. Ein Schraubenbruch nach einem Jahr konnte bei einer Person gesehen werden. Ein lokaler Infekt trat bei zwei Patienten unmittelbar postoperativ auf.

\section{perioperative Komplikationen und Spätfolgen nach KDA-OSG und Kompression-OSG}

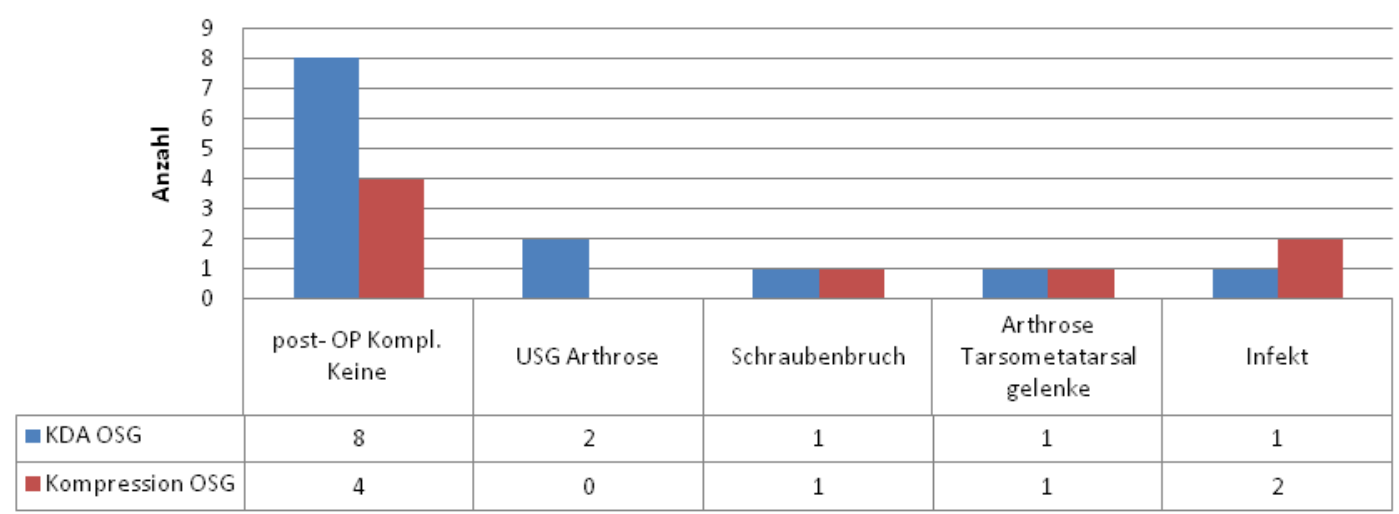

Abb.44: perioperative Komplikationen und Spätfolgen 


\subsubsection{Schmerzen nach OP}

\section{KDA-OSG}

Bei den OSG versteiften Patienten durch KDA gibt es nach der Operation noch dauerhaft bei 3 Patienten keine Schmerzen, 4 Patienten (30,77\%) berichten über leichte Schmerzen, in zwei Fällen erträgliche Schmerzen, bei einem Patienten starke Schmerzen und bei 3 Personen gibt es Anlaufschmerzen.

\section{Kompressions-Arthrodese-OSG}

In dem Kollektiv der Kompressions-Arthrodesen am OSG gibt es keinen Patienten ohne Schmerzen. Einen leichten Schmerz haben zwei Personen. Erträgliche Schmerzen gibt es bei zwei Patienten. Bei einer Person kommen noch dauerhaft starke Schmerzen vor. Drei Patienten schildern, dass sie unter Anlaufschmerzen leiden.

Eine statistische Signifikanz lässt sich nicht ermitteln.

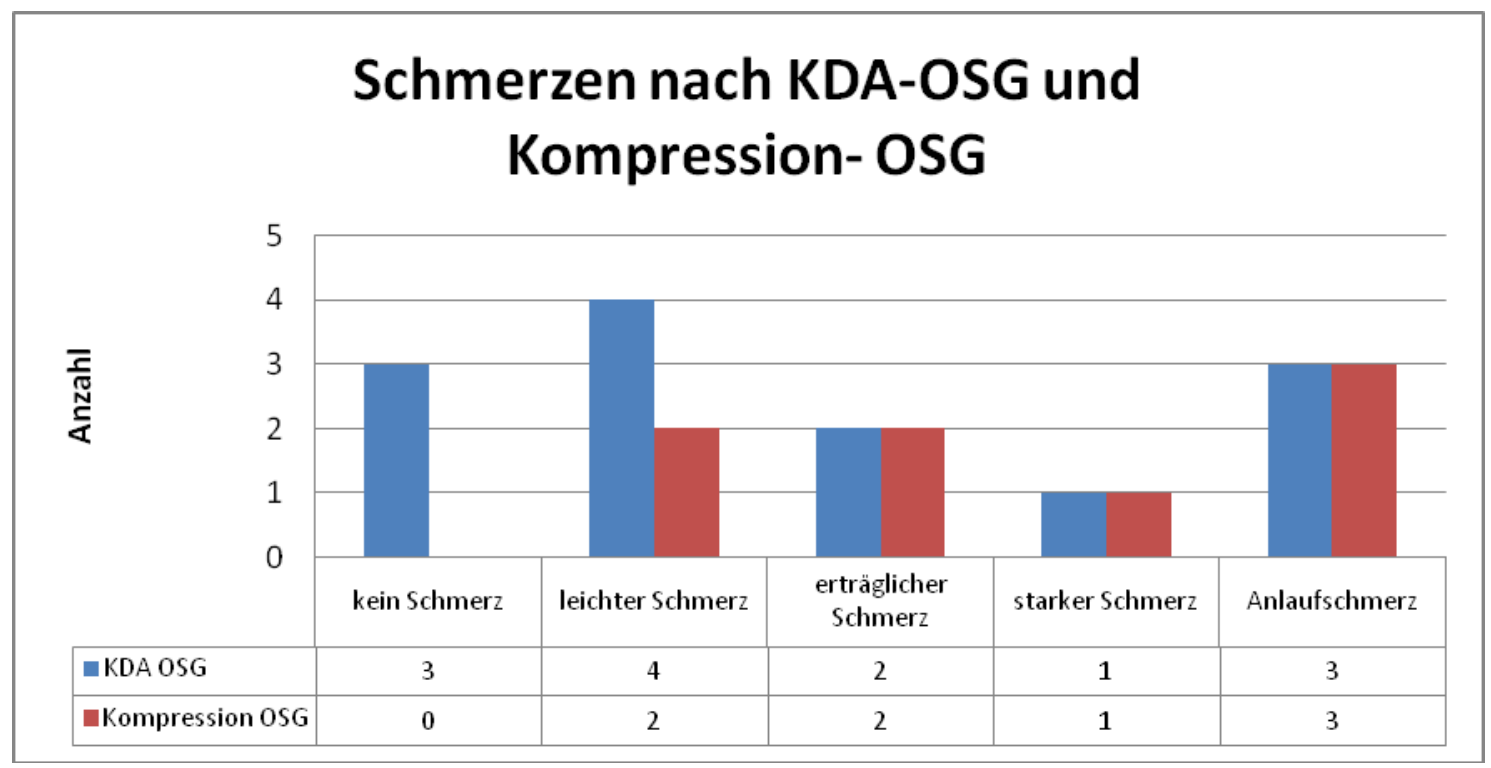

Abb.45: Schmerzen 


\subsubsection{Belastungsabhängige Schmerzen nach OP}

\section{KDA-OSG}

3 Patienten (23,08\%) haben nie Schmerzen unter Belastung. Weitere 5 Personen (38,46\%) berichten über gelegentliche Schmerzen unter Belastung. Weitere 3 Personen (23,08\%) haben kontinuierlich Schmerzen unter Belastung. Ruhe oder Spontanschmerz haben 2 Personen.

\section{Kompressions-Arthrodese-OSG}

3 Patienten (37,5\%) haben gelegentlich Schmerzen unter Belastung. Ebenfalls 3 Personen (37,5\%) geben an kontinuierlich Schmerzen unter Belastung zu haben. 2 Patienten haben Ruhe- oder Spontanschmerzen.

Bei der Betrachtung der belastungsabhängigen Schmerzen in beiden Gruppen sind keine signifikanten Unterschiede festzustellen.

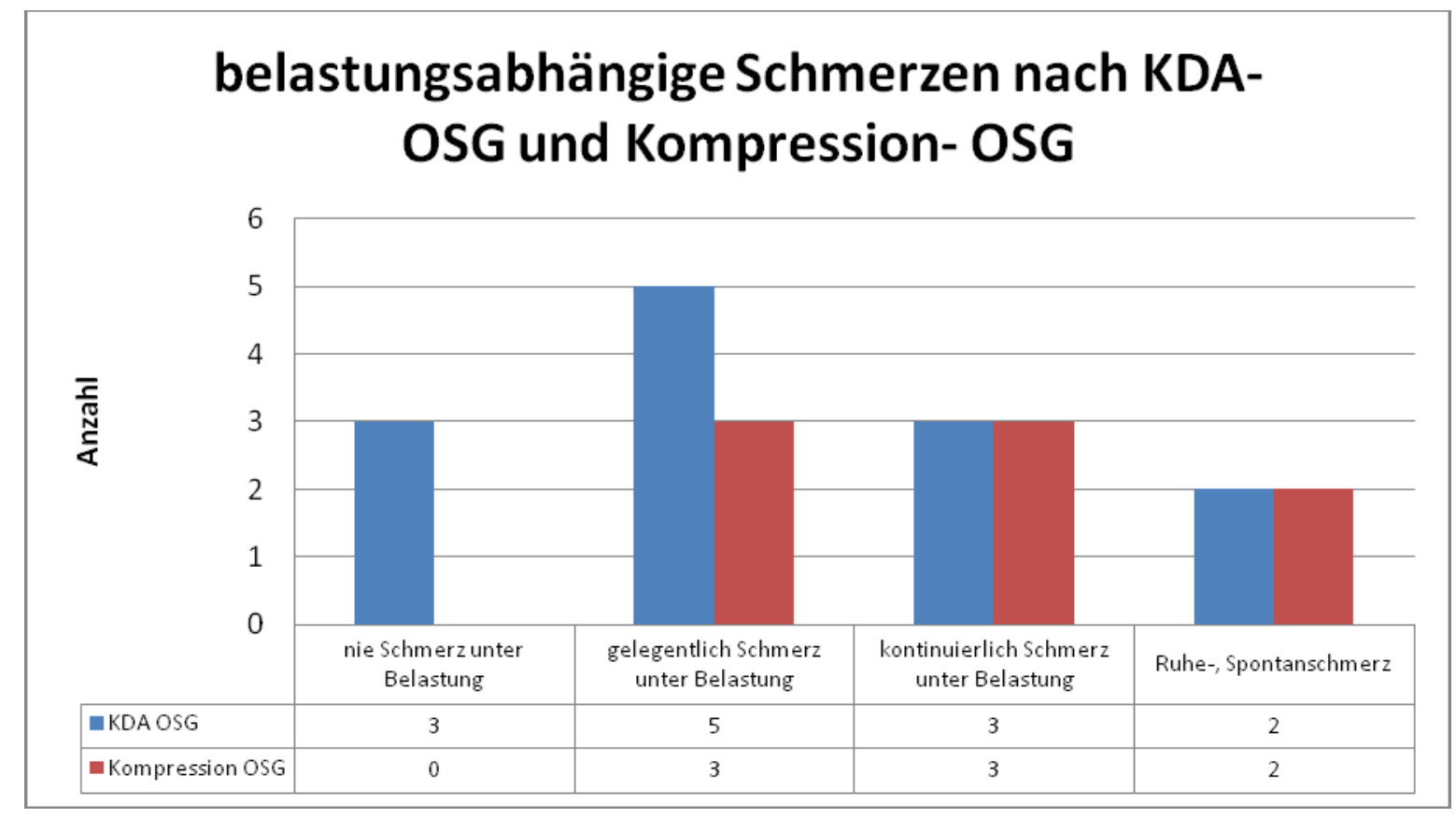

Abb.47: belastungsabhängige Schmerzen 


\subsubsection{Dauerhafte Schmerzmitteleinnahme nach OP}

\section{$\underline{\mathrm{KDA}-\mathrm{OSG}}$}

In der KDA-Gruppe nehmen 8 Patienten (61,54\%) nie Schmerzmittel ein. 4 weitere Personen $(30,77 \%)$ nehmen 1-2 Mal pro Woche Schmerzmittel. Nur ein Patient berichtet über eine tägliche Schmerzmitteleinnahme.

\section{Kompressions-Arthrodese-OSG}

In dem Vergleichskollektiv nehmen jeweils 2 Patienten nie, 1-2 Mal pro Tag, 3-5 Mal pro Tag und täglich Schmerzmittel.

Ein statistisch signifikanter Unterschied in der Schmerzmitteleinnahme zwischen KDA- und Kompressions-Gruppe ist nicht zu beobachten.

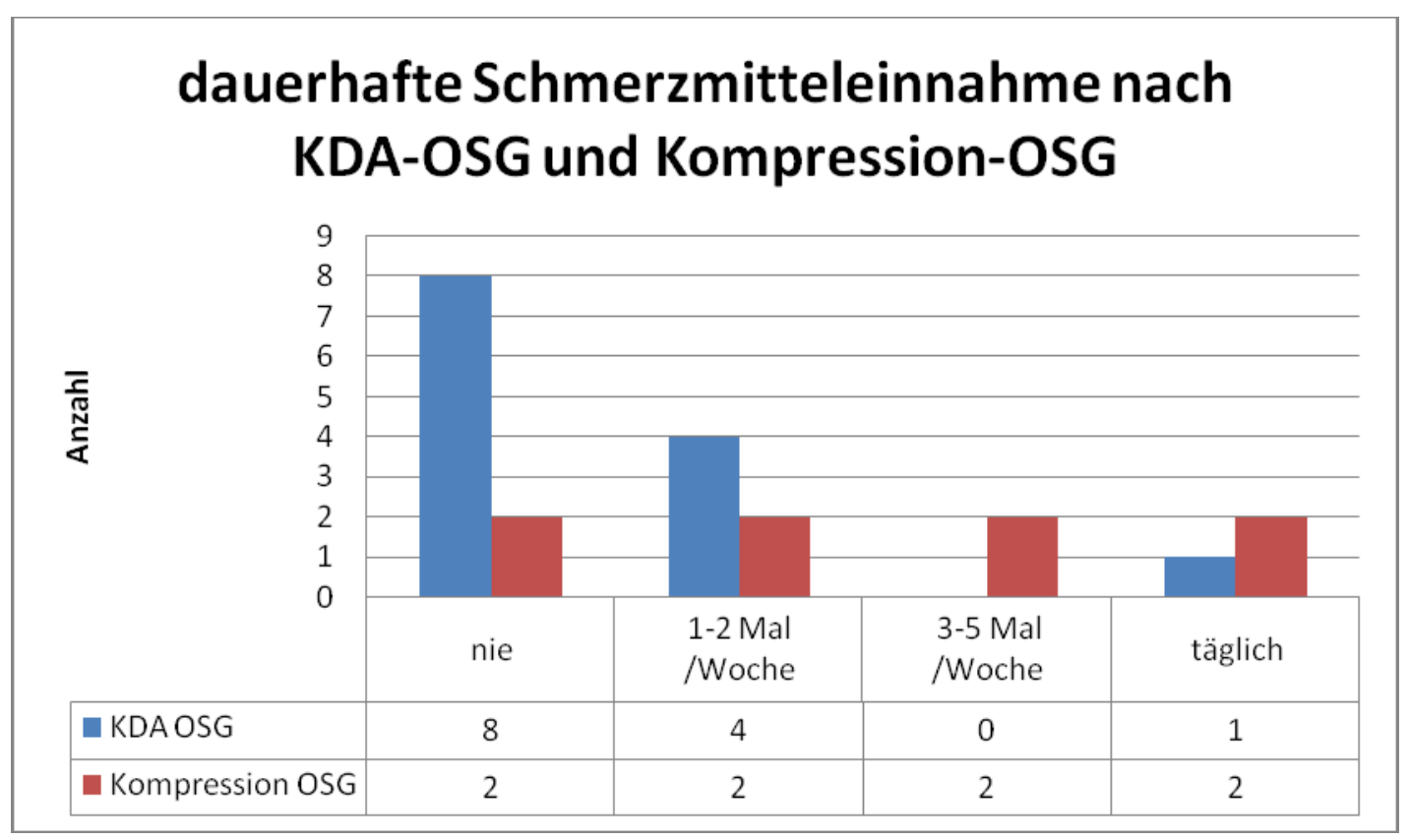

Abb.48: Schmerzmitteleinnahme 


\subsubsection{Schmerzfreie Gehstrecke nach OP}

\section{$\underline{\text { KDA-OSG }}$}

In der KDA-Gruppe gibt es drei Patienten, die schmerzfrei ohne

Einschränkungen gehen können. Weitere 4 Personen $(30,77 \%)$ sind in der Lage zwei Stunden ohne Schmerzen gehen zu können. Eine schmerzfreie Gehstrecke von einer Stunde haben zwei Personen. Ebenfalls zwei Patienten können 30 Minuten schmerzfrei unterwegs sein. In zwei Fällen wird berichtet, dass eine Schmerzfreiheit nur durch Gehen in Etappen mit Pausen zu erreichen ist.

\section{Kompressions-Arthrodese-OSG}

In dieser Gruppe ist kein Patient, der schmerzfrei ohne Einschränkungen gehen kann. Ein Patient kann hier zwei Stunden gehen ohne Schmerzen zu haben. Ohne Schmerzen zu haben kann eine Person 30 Minuten gehen. In 6 Fällen (75\%) ist ein schmerzfreies Gehen nur durch Etappen mit Pausen zu ermöglichen.

Hier ist kein statistisch signifikanter Unterschied zu beobachten.

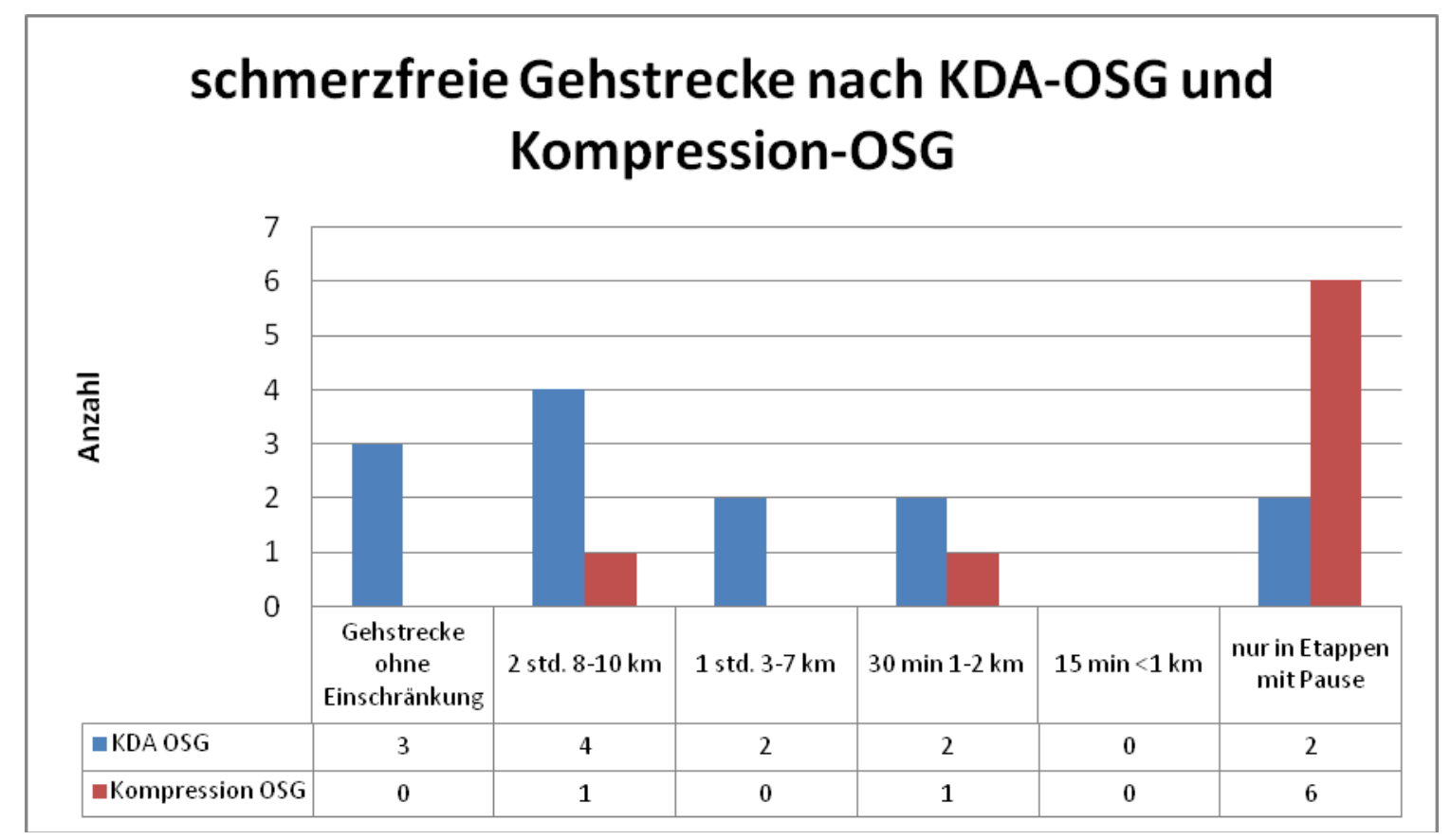

Abb. 49: schmerzfreie Gehstrecke 


\subsubsection{Subjektive Zufriedenheit}

\section{$\underline{\text { KDA-OSG }}$}

In der Gruppe der KDA am OSG gibt es keinen Patienten, der nur kaum zufrieden ist. Hier geben 4 Patienten $(30,77 \%)$ an leicht zufrieden zu sein. Sehr zufrieden sind 9 Patienten (69,23\%). In dieser Gruppe ist kein Patient unzufrieden.

\section{Kompressions-Arthrodese-OSG}

In dem Kollektiv mit Kompressions-Arthrodese am OSG gibt es drei Patienten, die nur kaum zufrieden sind. Weitere drei Personen geben an leicht zufrieden zu sein. Hier sind zwei Personen, die sehr zufrieden sind. Kein Patient ist unzufrieden.

Hiermit sind die Patienten, die eine KDA am OSG bekommen haben, statistisch signifikant zufriedener als die Patienten mit einer OSG-KompressionsArthrodese $(p=0,034)$.

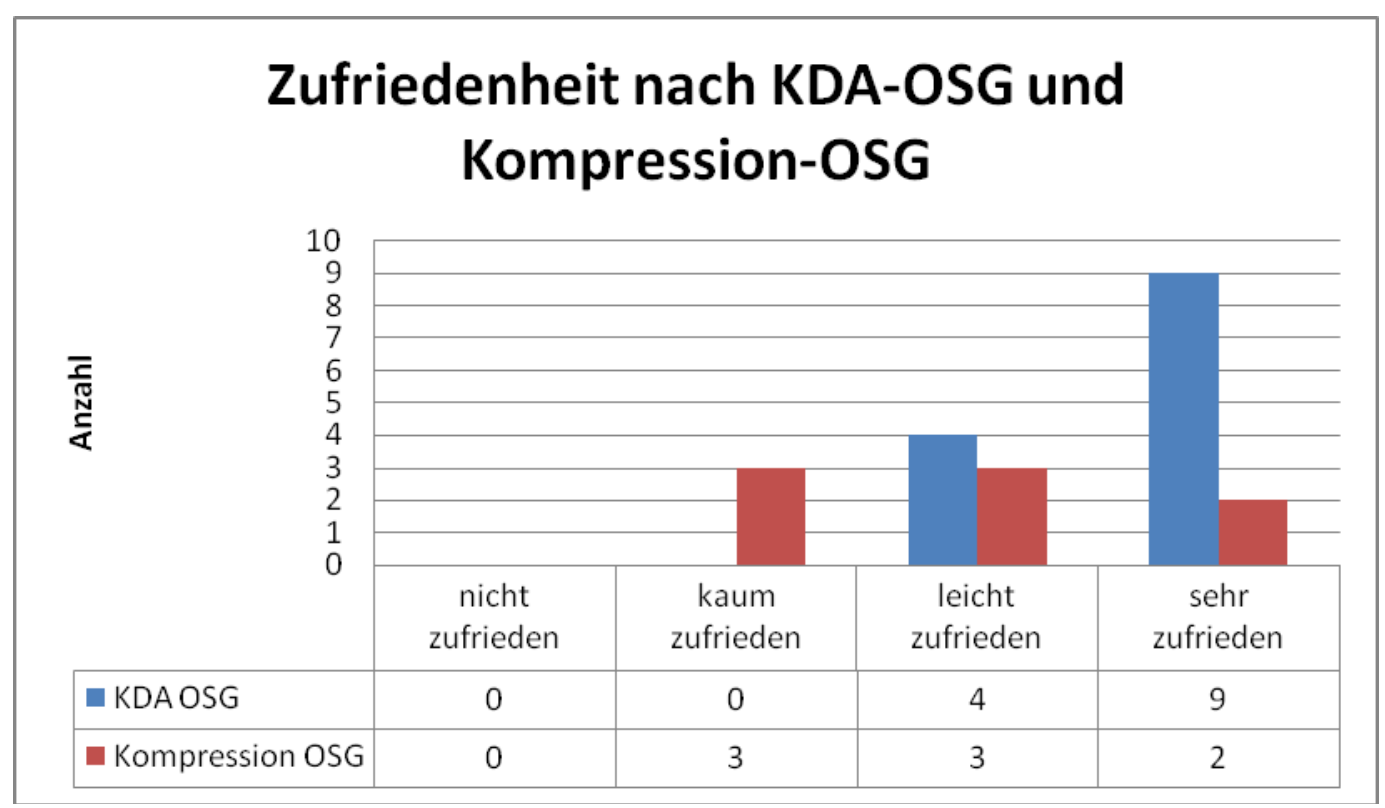

Abb.50: subjektive Zufriedenheit 


\section{Diskussion}

Die meisten Patienten haben einen langen Weg hinter sich, bevor die Indikation zur operativen Versteifung des Gelenks gestellt wird. Die Tatsache, dass es viele verschiedene Operationsverfahren zur Gelenkversteifung gibt, spricht dafür, dass keine optimale Methode existiert. Je mehr Methoden es gibt, desto schlechter ist meist die einzelne.

Die Frage nach der besten Operationstechnik ist nicht nur objektiv zu beantworten, sondern benötigt die subjektive Meinung des Patienten. Aus diesem Grund ist die Motivation dieser Arbeit entstanden, in der eben genau diese beiden Bereiche untersucht werden.

Oft klagen Patienten über postoperativ fortbestehende Schmerzen, eine durch die Arthrodese eingeschränkte Gehfähigkeit, oder eine allgemeine Unzufriedenheit.

Ziel der Arbeit ist es, verschiedene Verfahren zur Arthrodese des unteren und oberen Sprunggelenkes zu untersuchen.

Dabei ist es wichtig, eine Erkenntnis darüber zu erlangen, ob verschiedene Verfahren an den Gelenken OSG und USG unterschiedliche Ergebnisse erbringen.

Laut Statistischem Bundesamt verunfallen mehr Männer als Frauen. Dies zeigt sich in dem untersuchten Kollektiv: 24 Männer (69\%) standen 11 Frauen (31\%) gegenüber. Der Anteil an Männern im Berufsleben und am Straßenverkehr ist höher als der der Frauen. Insgesamt sind dadurch wesentlich mehr Männer in ein schweres Trauma verwickelt (Bardenheuer et al. 2000).

Lt. Literatur erhalten vor allem Männer eine Arthrodese (Ahlberg und Henricson 1981; Helm 1990; Mann et al. 1991; Papa und Myerson 1992; Chen et al. 1996; Buchner und Sabo 2003 a,b).

Das Alter der untersuchten Patienten liegt im Durchschnitt bei 42,65 Jahren für die Knochendübelarthrodese und bei 48,19 Jahren in der KompressionsArthrodese-Gruppe.

Insgesamt ist dies mit den Angaben in der Literatur vergleichbar. 
Ein Durchschnittsalter von Anfang bis Mitte vierzig ist häufig zu lesen (Scranton et al. 1980; Ahlberg und Henricson 1981; Boobbyer 1981; Schaap et al. 1990; Mann et al. 1991; Myerson und Quill 1991; Papa und Myerson 1992; Chen et al. 1996; Wülker et al. 1997).

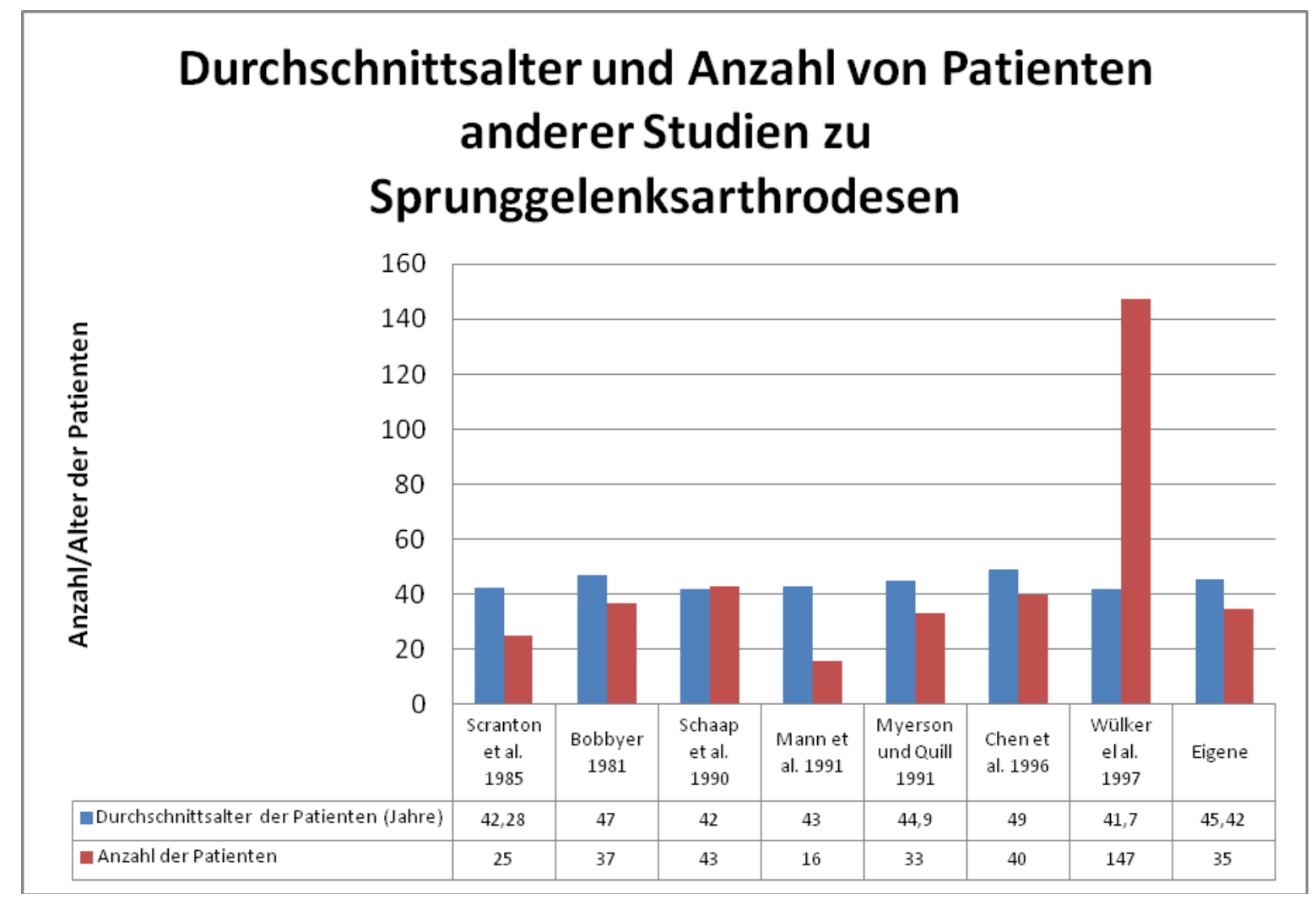

Abb.51: Durchschnittsalter von Patienten anderer Studien zu Sprunggelenksarthrodesen

Betrachtet man die vorausgehende Verletzung so finden sich in der vorliegenden Studie Patienten mit OSG-Frakturen $(n=6)$, Pilon-tibiale-Frakturen $(n=13)$ und Kalkaneusfrakturen $(n=12)$.

Diese drei primären traumatologischen Diagnosen machen in unserem Patientengut 88.6\% aller Patienten aus. Die OSG- bzw. Pilon-tibiale-Frakturen führten sekundär zu OSG-Arthrodesen, während nach Kalkaneusfrakturen USG-Arthrodesen durchgeführt wurden.

Das Risiko nach Frakturen am OSG und USG eine schmerzhafte posttraumatische Arthrose oder eine posttraumatische Pseudarthrose (meist 
nach primärer Osteosynthese) zu entwickeln ist in der Literatur beschrieben (Müller et al. 1999; Rehart et al. 1999; Thermann et al. 1999; Horn 2003; Jerosch und Vollmert 2005).

Die Arthrodese spielt vor allem bei posttraumatischen Arthrosen und Pseudarthrosen eine wichtige Rolle. Zahlreiche Autoren postulieren eine vornehmlichen Indikationsstellung zur Sprunggelenksarthrodese durch posttraumatische Arthrosen mit meist erheblicher Schmerzsymptomatik (Scranton et al. 1980; Boobbyer 1981; Moeckel et al. 1991; Myerson und Quill 1991; Wülker et al. 1997).

Die Arthrodese stellt somit die „ultima ratio“- Therapie für fortgeschrittene Sprunggelenksarthrosen dar. In der Literatur haben schon Scranton et al. (1980) über 81\% posttraumatische Arthrosen als Indikation für Sprunggelenksarthrodesen gesprochen.

Myerson und Quill (1991) haben in ihrer Studie 33 Arthrodesen nachuntersucht von denen $75 \%$ die posttraumatische Arthrose als Indikation hatten. Chen et al. (1996) hatten von 40 nachuntersuchten Patienten bei 18 Patienten (45\%) primär eine posttraumatische Arthrose.

In der vorliegenden Arbeit, mit 35 Patienten, befanden sich 24 Patienten mit einer schmerzhaften posttraumatischen Arthrose (68,6\%). Gefolgt wurde dies von 8 Personen (22,9\%), die wegen einer Pseudarthrose operiert wurden. Da es schwierig ist, die Operationsindikation auf eine einzige Diagnose zu bringen, wurde die maßgebliche Problematik in den Vordergrund gestellt. 1985 wurde das erste Mal eine große Nachuntersuchungsstudie von OSGArthrodesen durchgeführt. In dieser Studie wurden 101 Patienten mit derselben OP-Technik versorgt. Dabei lag der Nachuntersuchungszeitraum zwischen 5 und 25 Jahren. Bei allen Patienten wurden die Gelenkoberflächen von Talus und Tibia erhalten und das Gelenk mit 2 gekreuzten, transmalleolären Schrauben fixiert. Schon damals konnte eine Fusionsrate von 96\% erreicht werden. Nach der Operation wurde ein Unterschenkelgips ohne Belastung für 6 
Wochen angelegt. Anschließend bekamen die Patienten dann einen Unterschenkelgehgips für weitere 6 Wochen (Morgan et al. 1985).

In der Literatur finden sich zahlreiche Studien zu OSG- bzw. USG-Arthrodesen, jedoch mehr zu Arthrodesen des oberen Sprunggelenks. Gründe dafür können die primären traumatologischen Ursachen sein, die bei einer OSGGelenkbeteiligung entsprechend schmerzhaft arthrotische Gelenkveränderungen provozieren können und dann sekundär die Option einer operativen Arthrodese entstehen lassen. In unserem Kollektiv kommen mehr OSG-Arthrodesen (21 Fälle) als USG-Arthrodesen (14 Fälle) vor.

Dies entspricht dem in der Literatur vorherrschenden Trend von mehr OSG- als USG-Versteifungen.

In dieser Studie wurde ein Kollektiv von Patienten gewählt, die ausschließlich aus traumatologischen Gründen eine Arthrodese bekamen. Zwei häufige, in der Unfallchirurgie Göttingen, angewandte Verfahren stellten sich in den Vordergrund. Zum einen die Knochen-Dübel-Arthrodese (KDA) und zum anderen die Resektions-Kompressions-Arthrodese.

Aufgrund der Tatsache, dass seit Albert im Jahre 1879 über 100 verschiedene und modifizierte Arthrodeseverfahren entwickelt wurden, ist ein direkter Vergleich von eigenen Daten mit denen aus der Literatur nur schwer möglich. Ein nicht unerheblicher Teil der unfallchirurgischen Kliniken verwendet abgewandelte Operationstechniken. Die Ursache dafür kann in der individuellen Ausbildung bzw. Vorlieben der entsprechenden Operateure gesehen werden.

Allerdings wird häufiger von der Kompressions-Arthrodese am OSG nach Zimmermann durch Zugschrauben berichtet. Von dieser KompressionsArthrodese lässt sich in abgewandelter Form häufiger lesen (Lance et al. 1979; Scranton et al. 1980; Ahlberg und Henricson 1981; Hagen 1986; Kitaoka 1991; Stuhler 1994; Wülker et al. 1997).

Bei der Evaluierung der Operationsergebnisse ist es sinnvoll subjektive und objektive Daten zusammen zu betrachten. Hierzu wurden die drei oben genannten Scores und die Angaben von v.a. Zufriedenheit und Schmerzen betrachtet. In der Literatur wird bisweilen bei den Ergebnissen von sehr gut 
(excellent), gut (good), befriedigend (fair) und schlecht (poor) gesprochen. Nicht selten lassen diese Autoren Ergebnisevaluierungen durch Scores etc. vermissen (Boobbyer 1981; Morgan et al. 1985).

Bei der Evaluierung der Sprunggelenksverletzungen sowie des Fußes wird der Score von Mazur et al. 1979 häufig gebraucht (Dennis et al. 1990; Helm 1990; Schaap et al. 1990; Anderson et al. 2005).

Neben diesem Score von Mazuret al. 1979 (Boston children's Hospital ankle scoring system) wurde der Score von Kitaoka et al. 1994 (Ankle-HindfootScore/AOFAS) und der McGuire et al. 1988 Score verwendet. Der It. Literatur am häufigsten verwendete Score ist der Kitaoka et al. 1994 Score (Rehart et al. 1999; Trnka et al. 2001; Buchner und Sabo 2003 a,b; Horn 2003; Greisberg et al. 2004; Anderson et al. 2005; Fluckiger und Weber 2005; Trieb et al. 2005; Vienne 2005; Hopgood et al. 2006).

In der vorliegenden Auswertung sind bei der Gegenüberstellung von KDA und Kompressions-Arthrodese, unabhängig von OSG oder USG, bessere Ergebnisse bei den KDA Patienten zu sehen. Obwohl in der KDA-Gruppe die Patienten im Schnitt etwas jünger sind und das Kollektiv eine etwas größere Anzahl an Fällen aufweist. Diese besseren Ergebnisse korrelieren gut mit der in der KDA-Gruppe vorherrschenden etwas höheren Zufriedenheit.

Der Vergleich der beiden Kollektive von Patienten mit KDA bzw. KompressionsArthrodese bleibt ohne signifikante Unterschiede.

Bei der Betrachtung der KDA und Kompressions-Arthrodese am OSG können jedoch signifikante Unterschiede beobachtet werden.

Hierbei fallen alle drei Scores bei der Gruppe mit den KDA-OSG-Patienten deutlicher besser aus, als bei den OSG-Kompressions-Patienten.

Auf einem Signifikanzniveau von Alpha $=5 \%$ fallen die Unterschiede bei dem McGuire-Score sogar signifikant besser aus. Weiter ist zu beobachten, dass dieses signifikante Ergebnis bei dem objektiven Score in der subjektiven Zufriedenheit bestätigt wird. 
Patienten mit einer KDA am OSG sind signifikant zufriedener, als Patienten mit einer OSG-Kompressions-Arthrodese.

Signifikante Gründe hierfür können nicht nachgewiesen werden. Zu sehen ist allerdings, dass in der OSG-KDA-Gruppe die Schmerzen bzw. die schmerzfreie Gehstrecke besser ausfallen, als in der OSG-Kompressions-Gruppe. Dies kann ein Grund für die höhere Zufriedenheit in der OSG-KDA-Gruppe sein.

Bei der Untersuchung, ob die postoperativen Ergebnisse bei der KDA am oberen oder aber am unteren Sprunggelenk Unterschiede aufweisen, zeigen sich signifikante Ergebnisse.

In der Gruppe der OSG-KDA sind deutlich bessere Score-Werte als beim unteren Sprunggelenk mit KDA zu finden. Die Ergebnisse sind bei dem KitaokaScore im OSG sogar signifikant besser als beim USG.

Dieser objektiv positive Trend zu Gunsten der OSG-KDA kann hier im Bereich der subjektiven Zufriedenheit bestätigt werden.

Hiernach sind die Patienten mit einer KDA am OSG signifikant zufriedener als diejenigen mit einer KDA am USG.

Bei der Interpretation sollte bedacht werden, dass zwischen beiden Kollektiven ein signifikanter Altersunterschied besteht.

\section{Das Durchschnittsalter von 38,87 $\pm 9,69$ [23-61] Jahren der Patienten mit} KDA am OSG ist signifikant geringer, als das durchschnittliche Alter von 47,12+11,13 [20-58] Jahren in der KDA-USG-Gruppe.

Das geringere Alter in der OSG-Gruppe könnte dadurch bedingt sein, dass die jüngeren Patienten eher OSG-Frakturen und Pilon-tibiale Frakturen etc. durch Sport-, Berufs- und Verkehrsunfälle erleiden, als ältere Menschen. Aus diesen Diagnosen ergeben sich die posttraumatischen Arthrosen, die zur Arthrodese führen können.

Bei älteren Menschen hingegen ist zu vermuten, dass bevorzugt Stürze im Haushalt oder bei der Gartenarbeit zu Verletzungen des Kalkaneus bzw. des 
unteren Sprunggelenkes führen. Hieraus kann gegebenenfalls die Indikation für eine USG-Arthrodese entstehen.

Über langfristig bestehende Schmerzen nach einer Arthrodese ist nur wenig zu lesen. Es wurde bei vielen Autoren die Kategorie Schmerzen nur als Bestandteil eines Scores berücksichtigt (Goebel et al. 2003; Fluckiger und Weber 2005; Eberl et al. 2006).

In manchen Arbeiten wurde ein Schmerzvergleich von präoperativ zu postoperativ vollzogen (Boack 2005; Klaue und Bursic 2005).

Thermann et al. (1999) verglichen die Schmerzen zwischen konservativen und operativen Therapien.

Über die Darstellung von u.a. Schmerzen bei der operativen Versteifung von Rückfußgelenken und OSG anhand eines Fragebogens prä- und postoperativ konnte bei Wülker et al. (1997) gelesen werden.

Von den KDA-Patienten haben immerhin fast die Hälfte mit 45,83\% postoperativ langfristig keine oder nur leichte Schmerzen. In der Kompressionsgruppe fällt dieses Ergebnis mit 36,36\% der Patienten mit leichten Schmerzen etwas schlechter aus.

Die schmerzfreie Gehstrecke nach Arthrodese wird nur selten validiert. Goebel et al. (2003) fragten ihre Patienten nach anterograder oder retrograder Marknagelarthrodese, ob eine schmerzfreie Gehstrecke >200 Meter möglich ist. Günter et al. (2002) untersuchten in einer Studie u.a. die beschwerdefreie Gehstrecke vor und nach Sprunggelenksarthrodese.

Fast 60\% (58,33\%) aller KDA-Patienten haben eine schmerzfreie Gehstrecke von länger als einer Stunde. Hier schneiden die Kompressions-Patienten mit einer geringeren schmerzfreien Gehstrecke deutlich schlechter ab. In diesem Kollektiv können lediglich 18,18\% länger als eine Stunde gehen.

Zu möglichen Komplikationsraten lassen sich einige Ergebnisse finden. Moeckel et al. (1991) berichten zum Beispiel bei Schraubenarthrodesen von einer postoperativen Komplikationsrate von 28\%. In einer Vergleichsgruppe mit Fixateur externe wird hier sogar von $61 \%$ Komplikationen berichtet. Als 
mögliche Komplikationen werden hier nicht oder späte Fusion, Stressfrakturen, Pininfekt oder Wundheilungsstörungen und Fehlstellung genannt.

Generell wird von bis zu 45\% Komplikationen durch Nichtfusion, Infekt und Fehlstellung etc. berichtet (Frey et al. 1994; Cooper 2001). Ein besonderes Problem nach einer Arthrodese stellt die Anschlussarthrose dar. Hierzu berichten Buchner und Sabo (2003 a), dass bei 47\% ihrer 48 nachuntersuchten Patienten nach durchschnittlich 9,3 Jahren eine Anschlussarthrose (subtalar) stattgefunden habe. Allgemein wird davon berichtet, dass nach genügend langer Nachuntersuchungszeit nach OSG-Arthrodesen v.a. Anschlussarthrosen des Subtalargelenkes auftreten können (Kitaoka 1991; Scranton 1991; Wülker et al. 1997; Zwipp et al. 1999).

Insgesamt wird berichtet, dass bei externen Verfahren deutlich mehr Komplikationen zu erwarten sind, als bei internen (Zwipp et al. 1999).

Diese, in der Literatur beschriebenen Zahlen, werden in dem untersuchten Kollektiv bestätigt. In dem Kollektiv von KDA-Patienten wird eine Rate an Komplikationen bzw. Folgeproblemen wie Anschlussarthrosen von 41,67\% beobachtet. Das bedeutet im Umkehrschluss, dass 58,33\% aller KDA-Patienten ohne jegliche Komplikationen und Anschlussprobleme leben können. In der Gruppe der Kompressions-Arthrodese Patienten sieht es dem allgemeinen Trend entsprechend etwas ungünstiger aus. Hier haben nur $45,45 \%$ der Patienten keine Komplikationen oder Anschlussprobleme. Bei der Betrachtung des OSG, kann festgestellt werden, dass hier die KDA im Vergleich zur Kompressions-Arthrodese mit 61,54\% komplikationslosen Fällen deutlich besser abschließt, als die Kompressions-Arthrodese-Patienten am OSG.

Die Frage ob die KDA am OSG häufiger ohne Komplikationen bleibt als am USG kann bejaht werden. 61,54\% der KDA des OSG bleiben ohne Komplikationen oder Folgeprobleme. Am USG kommt die KDA auf 54,55\% komplikationslose Fälle. All dies sind jedoch statistisch nicht signifikante Ergebnisse. Aber ein hinweisender Trend ist zu sehen. 
Bei einem Vergleich von offenen und arthroskopisch assistierten Arthrodesetechniken (im Folgenden AAA genannt) wird im Verlauf beobachtet, dass die Fusionsraten in etwa gleich sind.

Es muss allerdings festgestellt werden, dass Patienten, die arthroskopisch assistiert operiert wurden, einen geringeren Blutverlust, einen schnelleren Knochendurchbau und eine kürzere Krankenhausverweildauer hatten (Myerson und Quill 1991).

So kann gesagt werden, dass sich in der Literatur in der Hauptsache die (interne) Kompressions-Arthrodese und die Arthrodese mit Verriegelungsspan als Arthrodeseverfahren durchgesetzt haben (Mittelmeier et al. 1975; Mittelmeier und Nizard 1981; Dennis et al. 1990; Gruen und Mears 1991; Katcherian 1998).

\section{Kernaussagen:}

Anhand unserer Patientendaten kann geschlussfolgert werden, dass die KDA genauso gute bis bessere Ergebnisse liefert, als die Kompressions-Arthrodese.

Am OSG verdeutlicht sich dieser Trend.

Es wird beobachtet, dass die KDA am OSG besser abschneidet, als am USG.

Die Anwendung der KDA im Vergleich zur Kompressions-Arthrodese ist daher durchaus zu empfehlen.

Wenn es darum geht, das OSG zu versteifen, ist nach unserer Datenlage der KDA den Vortritt zu lassen.

Im Vergleich zum Kompressions-Arthrodese-Kollektiv zeigt sich bei den KDAPatienten, dass die Ergebnisse im Hinblick auf die postoperativen ScoreErgebnisse, die Schmerzen, die schmerzfreie Gehstrecke und die Zufriedenheit besser ausfallen. 


\section{Zusammenfassung}

Patienten, die eine Fraktur mit Gelenkbeteiligung erleiden, haben ein hohes Risiko, im Verlauf eine posttraumatische Arthrose zu entwickeln. Das Risiko steigt proportional mit der Ausprägung des Traumas. Je umfangreicher und anspruchsvoller die rekonstruierende Operation ist, desto höher wird das Risiko.

Eine manifeste posttraumatische Arthrose bedeutet für den Patienten Schmerzen, eingeschränkte Mobilität und damit eine berufliche und private Isolation.

Eine operative Gelenksversteifung ist die „ultima ratio“ um eine Reduktion der Schmerzen zu erreichen. Auf Kosten einer Bewegungseinschränkung ist es möglich, die beschwerdefreie Mobilität bis hin zu sportlichen Aktivitäten zu erhöhen.

Das bedeutet für den Patienten, dass eine Teilnahme am Leben (beruflich und privat) besser möglich ist.

Ziel der Arbeit ist es, ein Patientenkollektiv von 35 Personen mit entweder einer KDA oder einer Resektions-, Kompressionsarthrodese am OSG oder USG zu analysieren.

Die Studie sollte subjektive und objektive Ergebnisse liefern, die eine vergleichende Analyse der Operationsverfahren an den Sprunggelenken ermöglicht.

In einer Filemaker Datenbank wurden Patientendaten aus Akten, Fragebögen und klinischer Untersuchung gesammelt. 
Es wurde an 73 Patienten ein Fragebogen geschickt. Davon sind:

- 6 verstorben

- 1 Hospiz

- 1 Kur

- 4 nicht bereit zur Teilnahme

- 16 unbekannt verzogen

- 45 Fragebögen wurden zurückgesandt.

Von den 45 Personen, die den Fragebogen zurück gesendet haben, wurde mit 40 telefonisch ein Untersuchungstermin vereinbart. 5 Patienten wurden aufgrund von einer Entfernung $>300$ km nicht untersucht.

Unter diesen 40 Personen waren 35, die entweder eine isolierte OSG- oder USG-Arthrodese bekommen haben.

Der Fragebogen orientierte sich im Grundsatz an den Bereichen:

- Schmerz

- Subjektive Zufriedenheit

- Gehhilfen

- Gehstrecke

- Schmerzmitteleinnahme

- Zehenspitzenstand

- Treppensteigen

- Gehprobleme.

Anhand dreier Scores konnten die Ergebnisse dargestellt werden.

An ihnen wird die postoperative Situation auf einer Scala von 0-100 Punkten dargestellt, von „schlecht“ bis „sehr gut".

Bei der statistischen Auswertung wurden kategorielle Variablen zwischen den Operationsgruppen bzw. den Spunggelenksstellen mit dem Chi-Quadrat-Test analysiert. Die ordinalen und metrischen Daten wurden mit dem Mann-WhitneyU-Test untersucht. Die kategoriellen Ergebnisse wurden prozentual und mit 
Hilfe von Balkendiagrammen dargestellt, die Score-Werte und die Altersverteilungen wurden durch Boxplots präsentiert. Als Signifikanzniveau wurde Alpha $=5 \%$ festgelegt.

Unter den 35 analysierten Patienten sind 24 (68,6\%) mit einer KDA-Arthrodese und $11(31,4 \%)$ mit einer Resektions-, Kompressionsarthrodese versorgt worden. Es standen 24 Männern (69\%) 11 Frauen (31\%) gegenüber. 21 Patienten (60\%) bekamen eine Arthrodese am OSG und 14 (40\%) eine am USG.

Von den 24 KDA-Arthrodesen sind 13 (54,2\%) OSG-Versteifungen, während bei 11 Patienten $(45,8 \%)$ das USG versteift wurde.

Im Wesentlichen wurde folgendes analysiert:

1. KDA versus Resektions-,,Kompressionsarthrodese unabhängig vom Gelenk.

2. KDA am OSG versus KDA am USG.

3. KDA am OSG versus Resektions-, Kompressionsarthrodese am OSG.

Ergebnisse:

Zu 1. Ein Vergleich der beiden Kollektive bleibt ohne signifikante Unterschiede, obwohl ein positiver Trend zugunsten der KDA-Patienten zu sehen ist.

Zu 2. Die Analyse ergibt signifikante Unterschiede:

- Patienten mit einer OSG-KDA sind signifikant jünger, als Patienten mit USG-KDA.

- Patienten mit einer OSG-KDA erreichen im Kitaoka-Score signifikant bessere Ergebnisse, als Patienten mit USG-KDA. Die anderen beiden Scores fallen für die OSG-KDA hinweisend besser aus. 
- Die subjektive postoperative Zufriedenheit ist bei OSG-KDA-Patienten signifikant besser, als bei USG-KDA-Patienten.

Zu 3. Eine Analyse liefert folgende signifikante Unterschiede:

- Patienten mit einer OSG-KDA erreichen im McGuire-Score signifikant bessere Ergebnisse, als Patienten mit einer OSG-Resektions-, Kompressionsarthrodese.

- Die postoperative Zufriedenheit bei Personen mit einer OSG-KDA ist signifikant höher, als bei OSG-Resektions-, KompressionsarthrodesePatienten.

Abschließend kann gesagt werden, dass die Knochen-Dübel-Arthrodese ebenso gute bis bessere Ergebnisse liefert, als die Kompressions-Arthrodese.

Am oberen Sprunggelenk verdeutlicht sich dieser Trend.

Es ist festzustellen, dass die KDA am OSG besser abschneidet, als am USG.

Die Anwendung der KDA im Vergleich zur Kompressions-Arthrodese ist daher durchaus zu empfehlen.

Wenn es um eine OSG-Versteifung geht, ist der Vortritt nach unserer Datenlage der KDA zu gewähren. 


\section{Fallbeispiele}

\section{Fall 1:}

S.E., männlich, geb. 1952: KDA-

\section{OSG}

Primäre Diagnose:

Der Patient erlitt 1991 eine

traumatische

Sprunggelenksluxationsfraktur mit

Syndesmosenruptur rechts.

\section{OP 1:}

Osteosynthetische Versorgung der Sprunggelenksfraktur mit K-Draht und Draht-Cerclage 1991 (Abb.52).

Indikation zur Arthrodese:

Der Patient entwickelte nach der ersten Operation eine schmerzhafte OSG-Arthrose (Abb.53) sowohl in Ruhe, als auch bei Belastung.

\section{Röntgen:}

Es bestand eine OSG-Panarthrose mit ausgeprägten Knorpelschäden (Abb.53).

\section{OP 2:}

Nach diversen frustranen konservativen Therapieversuchen wurde im Februar 1998 eine Knochen-
Dübel-Arthrodese des OSG rechts durchgeführt. Nach Entnahme von 5 kortikospongiösen Knochendübeln (4x 12mm, 1x 11mm) aus dem Beckenkamm wurden diese in die vorgebohrten Transplantatlager eingebolzt. Zur Fixation wurden von lateral und medial jeweils 2 und von ventrolateral eine Schraube eingebracht (Abb.54/55).

Vollbelastung:

Nach 9 Wochen erfolgte die schmerzfreie Vollbelastung (Abb.56/57).

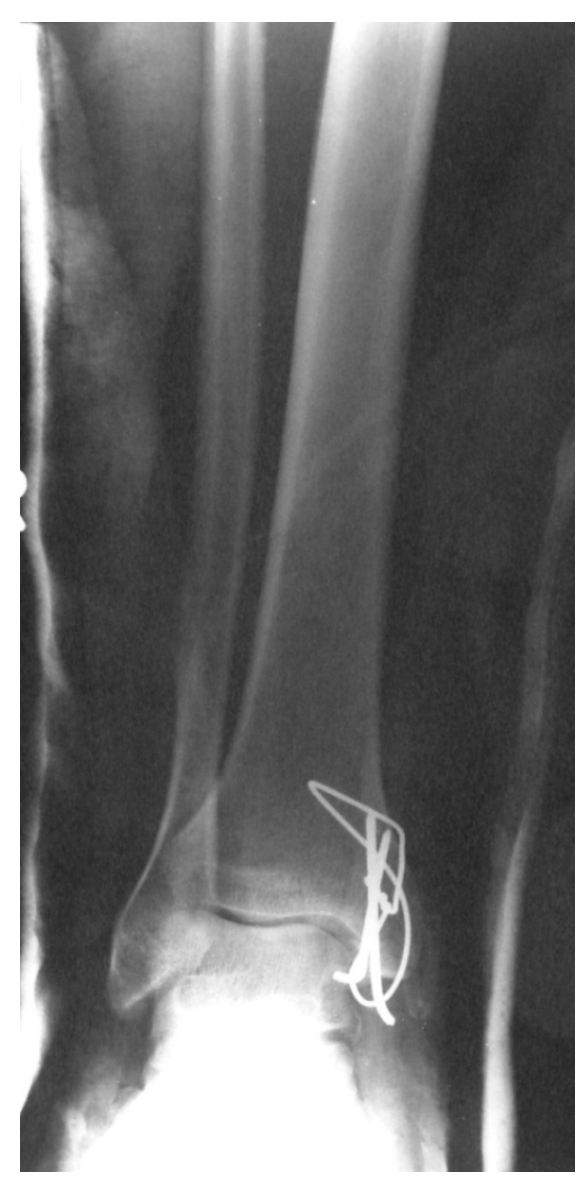

Abb.52: operativ versorgte OSGLuxationsfraktur 


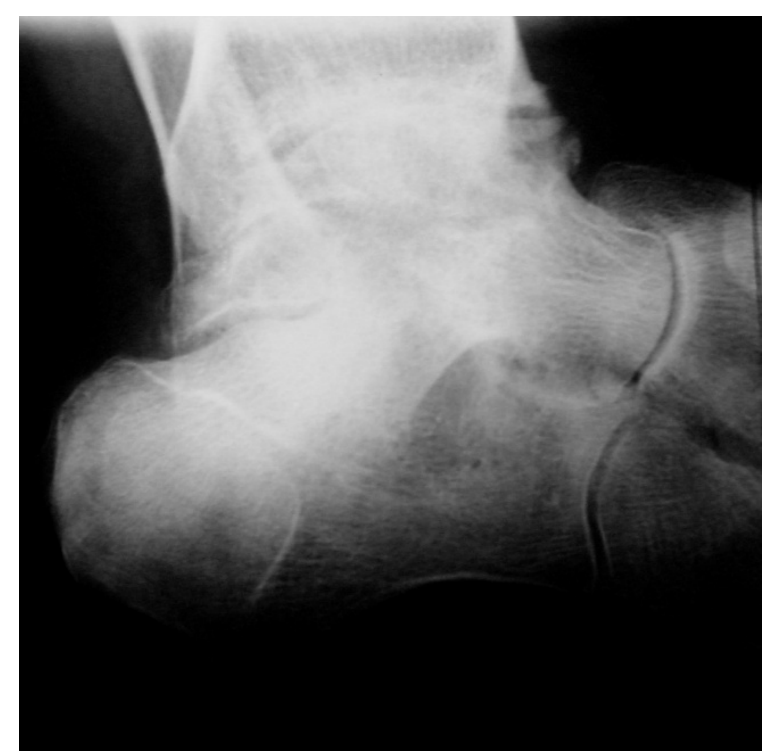

Abb.53 : posttraumatische OSG-Arthrose bei Z.n. OSG-Luxationsfraktur rechts

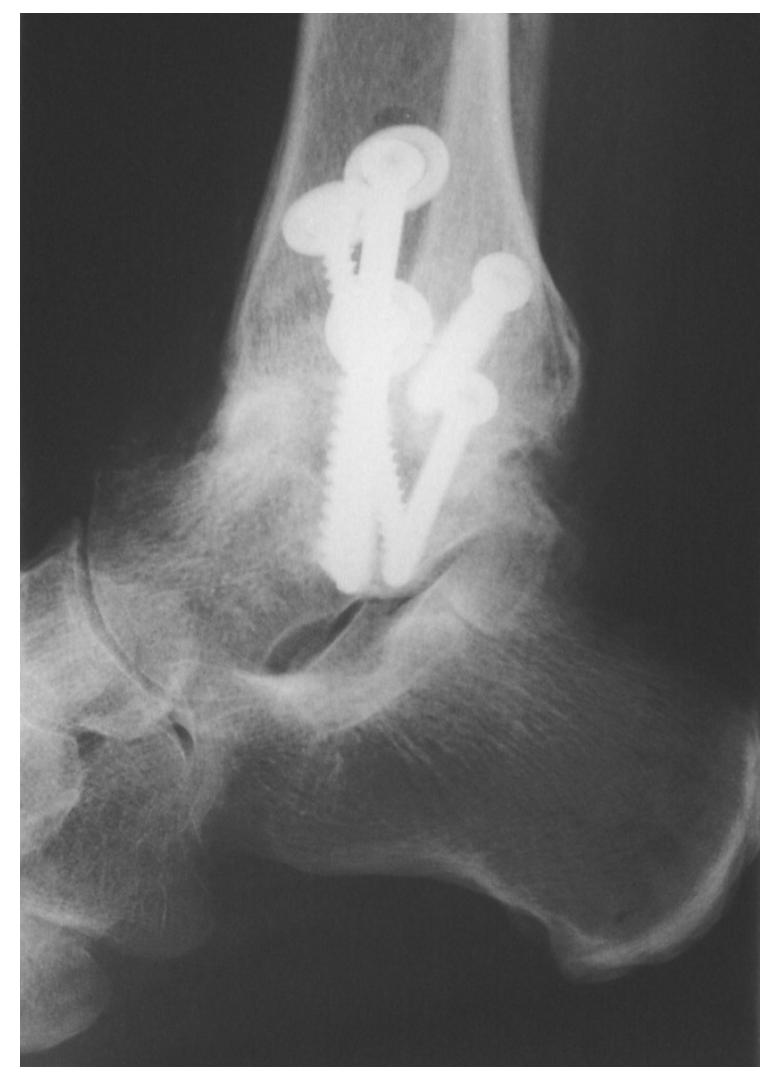

Abb.54: Z.n. OSG-KDA mit 5 eingebrachten Schrauben

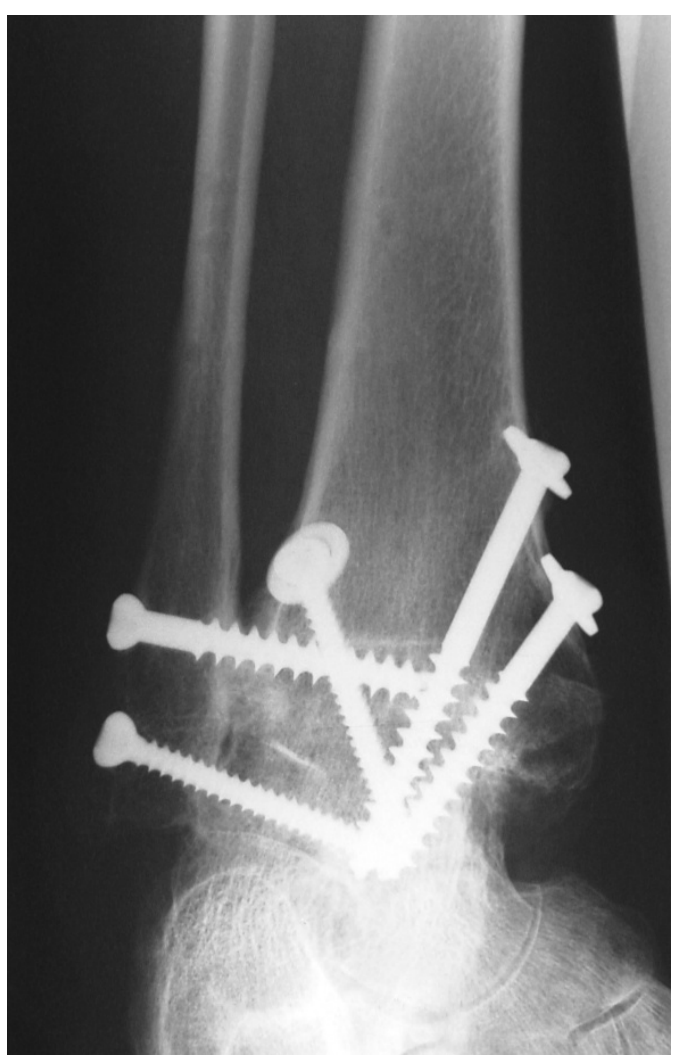

Abb.55: Z.n. OSG-KDA mit 5 eingebrachten Schrauben

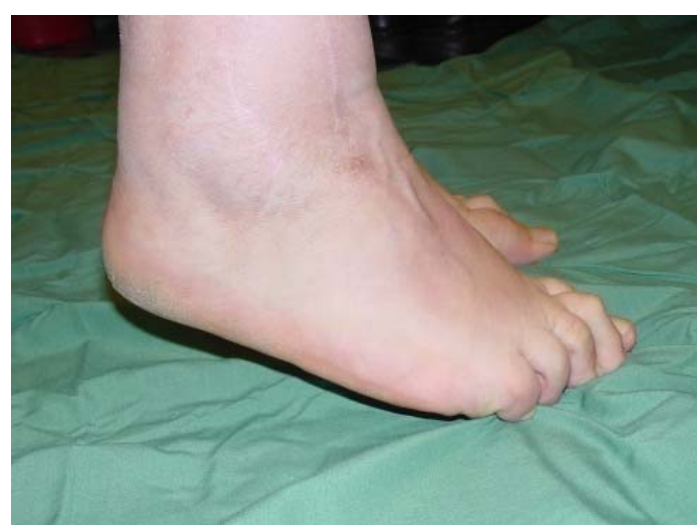

Abb.56: Zehenspitzenstand nach ausgeheilter OSG-KDA rechts 


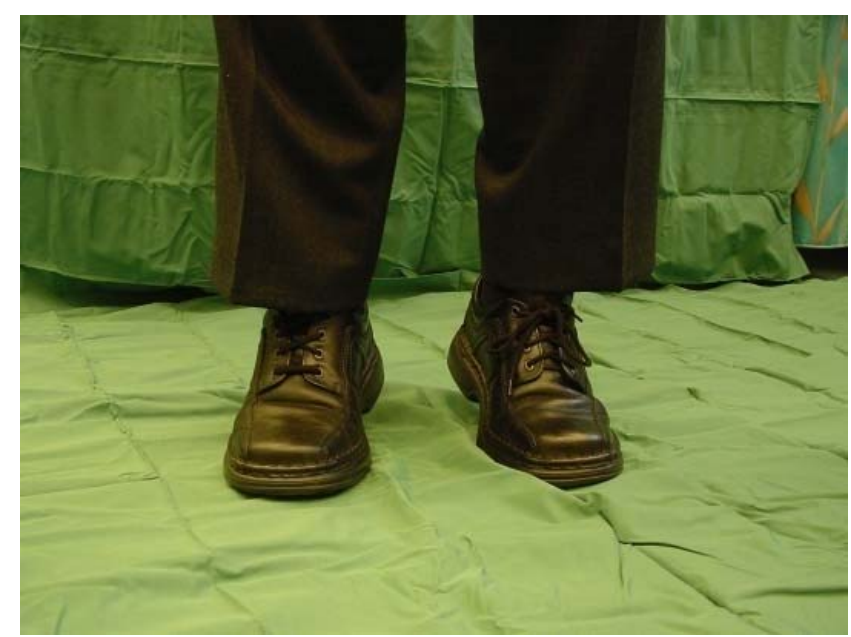

\section{Fall 2:}

Sch. F., männlich, geb.: 1959: KDA-

\section{$\underline{\text { USG }}$}

\section{Primäre Diagnose:}

Kalkaneusfraktur rechts 1993 durch

Sturz von der Leiter.

\section{OP 1:}

Initial osteosynthetische Versorgung der Kalkaneusfraktur (Abb.58).

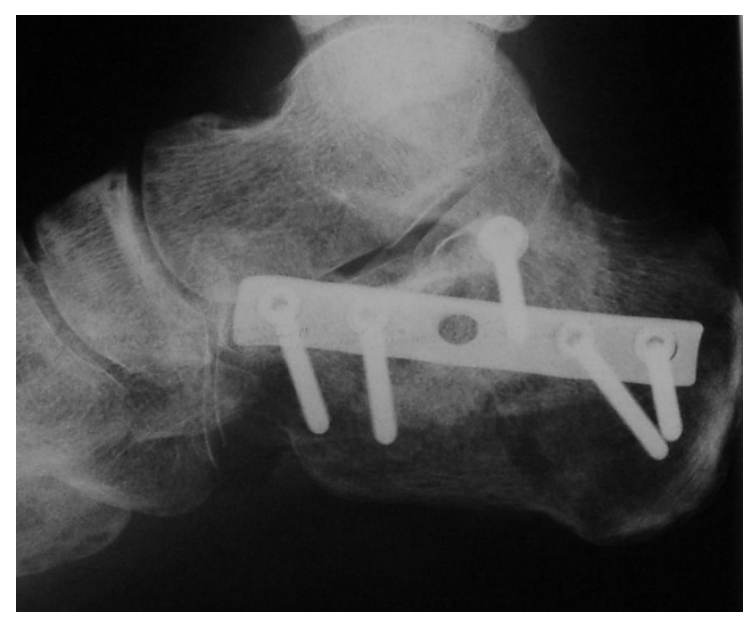

Abb.58: osteosynthetisch versorgte Kalkaneusfraktur
Abb.57: Zehenspitzenstand nach

ausgeheilter OSG-KDA rechts

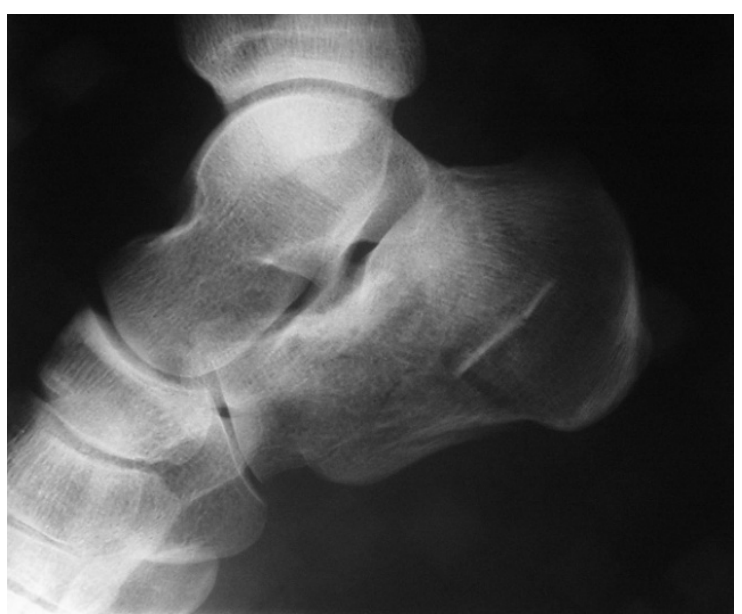

Abb.59: Z.n. osteosynthetisch versorgter Kalkaneusfraktur; nun mit erheblicher USGSchmerzhaftigkeit

Indikation zur Arthrodese:

Bestehen einer ausgeprägten USG-

Schmerzhaftigkeit und die

Schmerzfreiheit nach Infiltration von Lokalanästhetikum ins USG. 


\section{Röntgen:}

Radiologisch bestand keine

imponierende Arthrose (Abb.59).

\section{OP 2:}

KDA im Jahre 2000 (Abb.60-64).

Entnahme von 3x14 mm

Knochendübel und Spongiosa aus

dem rechten Beckankamm und

Einbolzung in die entsprechend

vorgebohrten Kanäle. (Abb.60-64)

Zur Kompression Einbringung von 3x

7,3 mm kanülierten Schrauben von

plantar (Abb.60).

Nachbehandlung:

Der Patient wurde ab dem 10.

postoperativen Tag in einer softcastlangschafts-Orthese mobilisiert. Die schmerzfreie Vollbelastung war ab der 8. postoperativen Woche bei radiologischer Konsolidierung möglich.

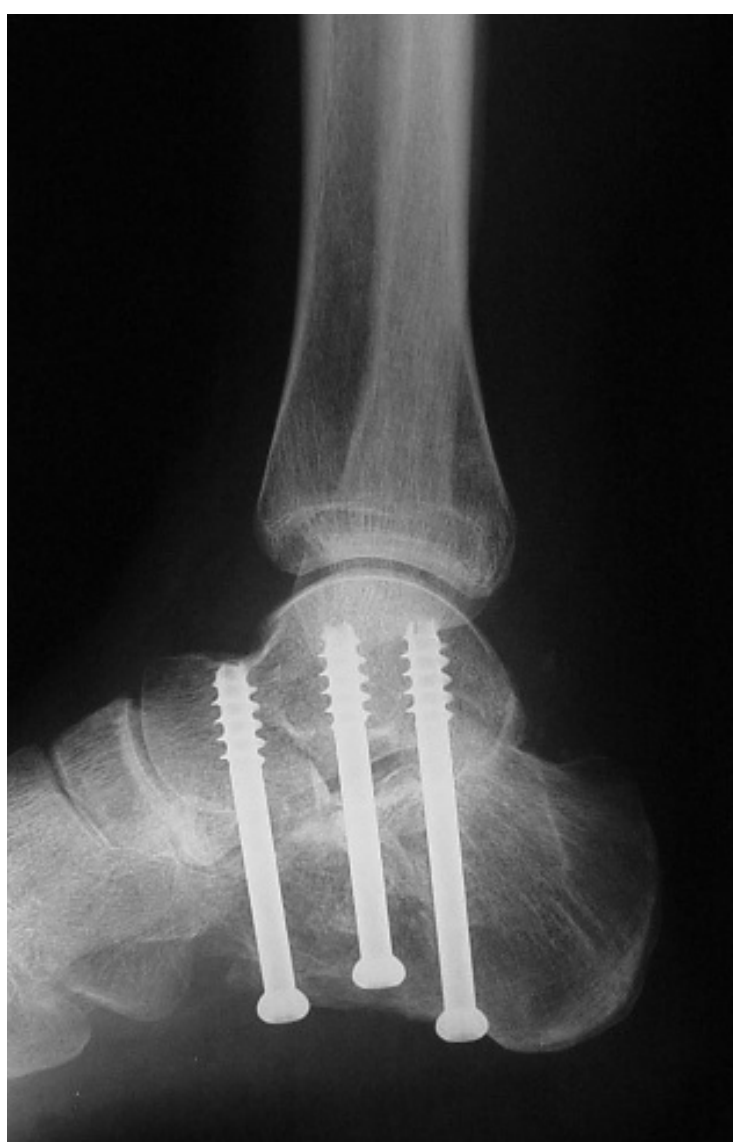

Abb.60: USG-Arthrodese mit 3 kanülierten

7,3 mm Schrauben

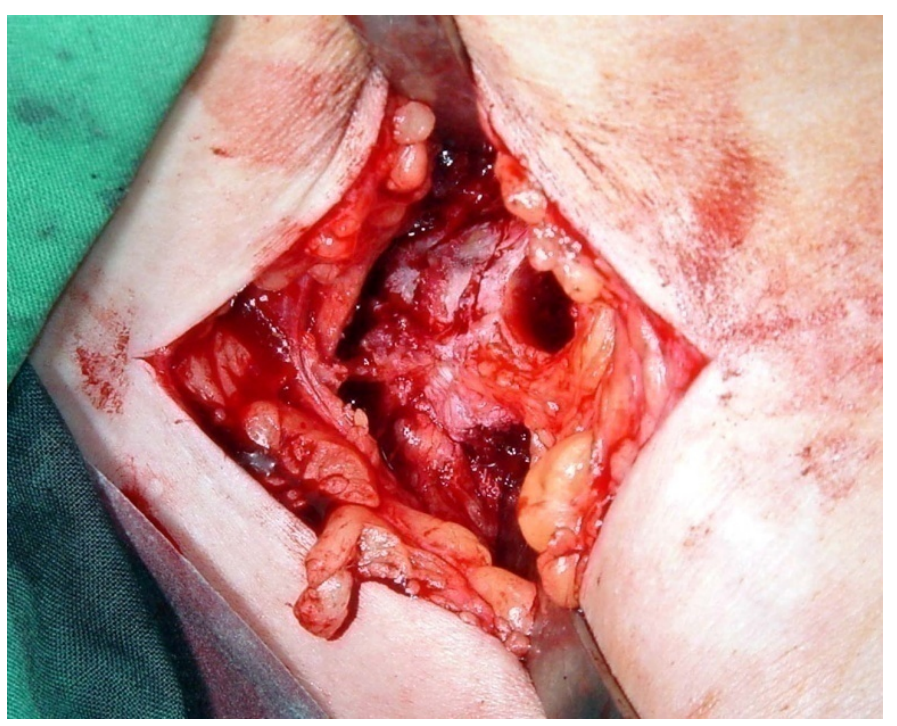

Abb.61: Entnahmestelle eines

Knochendübels am Beckenkamm 


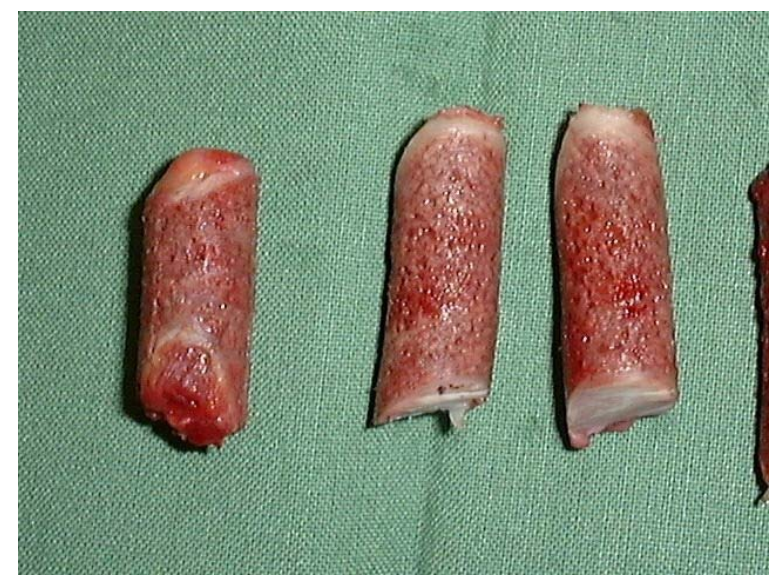

Abb.62: 3 entnommene Knochendübel aus dem Beckenkamm

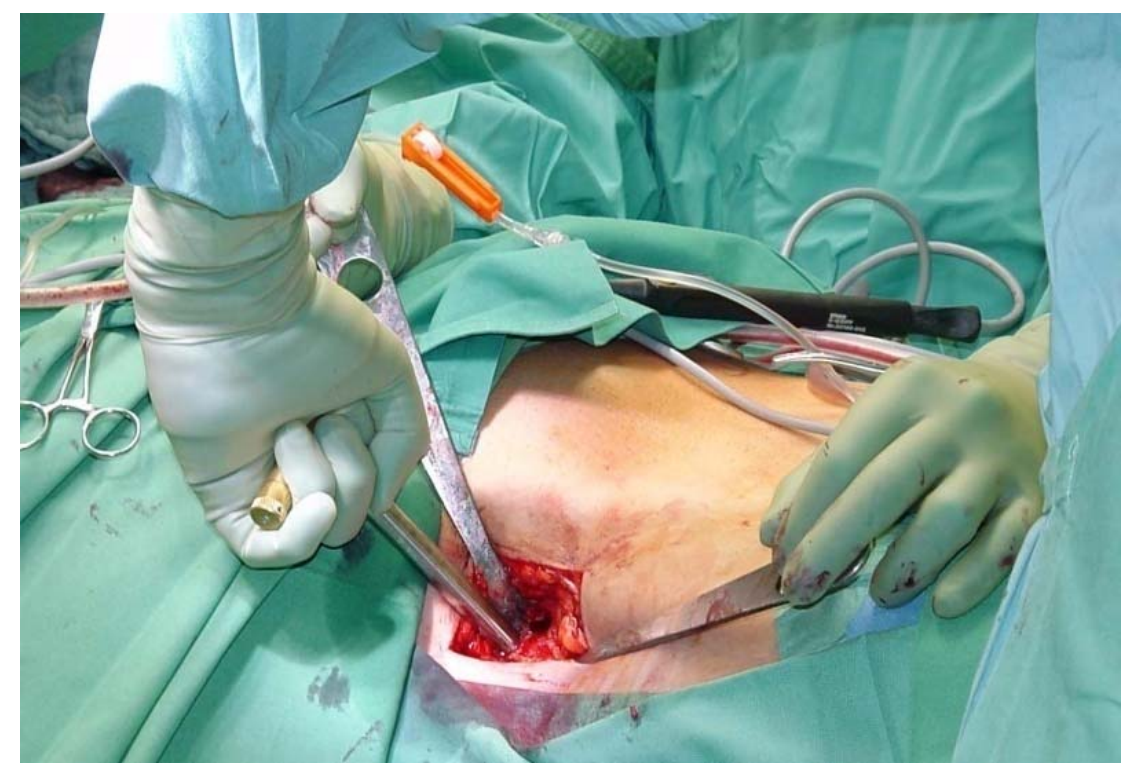

Abb. 63: Entnahme eines Knochendübels aus dem Beckenkamm

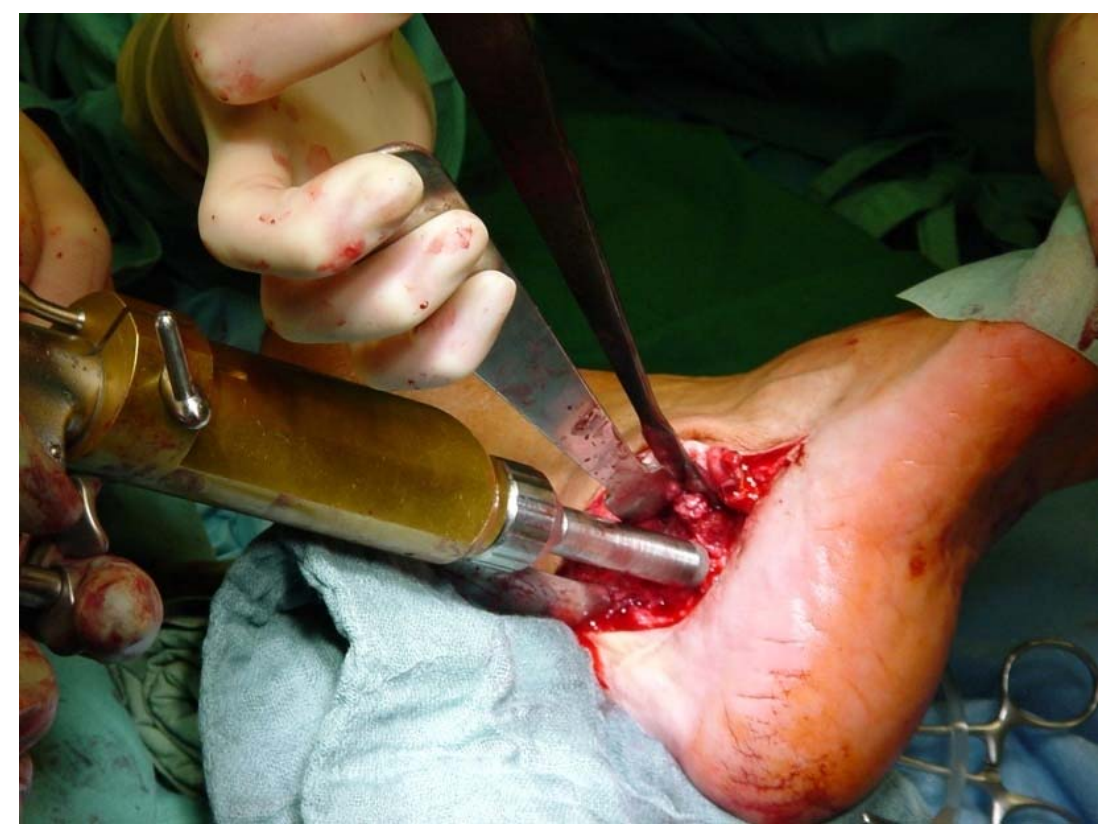

Abb.64: Fräsen eines Dübelkanales mit der DBCS-Fräse 


\section{Fall 3:}

J.B. weiblich, geb.: 1964: KDAOSG

Primäre Diagnose:

Traumatische trimalleoläre Pilontibiale Fraktur links im Jahre 2000.

\section{OP 1:}

Primär wurde eine chirurgische

Rekonstruktion und Osteosynthese des oberen Sprunggelenks durchgeführt.

\section{Röntgen:}

Radiologisch fand sich ein Jahr nach der primären OsteosyntheseOP ein fast aufgehobener Gelenkspalt, insbesondere lateral.

Indikation zur Arthrodese:

Aufgrund einer persistierenden und therapieresistenten

Schmerzsymptomatik im linken

Sprunggelenk, der massiven

Gelenkspaltverschmälerung und einer Schmerzreduktion nach Gelenksinfiltration mit Lokalanästhetikum wurde die Indikation zur Arthrodese gestellt.

\section{OP 2:}

Im Jahre 2001 wurde eine KDA am linken OSG durchgeführt. Es erfolgte eine Entnahme von $4 x$ $12 \mathrm{~mm}$ Knochendübeln aus dem Beckenkamm, die in die jeweils gefrästen Kanäle eingebolzt wurden. Zusätzlich wurde zur Stabilisierung und Kompression eine Zugschraubenosteosynthese (Spongiosaschraube) von fibular in Richtung Talus ventralseits durchgeführt.

\section{OP 3:}

Bei der Patientin bestand jedoch ein Jahr nach der Operation weiterhin eine Schmerzhaftigkeit im Bereich des linken oberen Sprunggelenks bei längerer Belastung.

Deshalb wurden trotz einer radiologisch festen und durchbauten Arthrodese zwei 7,3 mm Kompressionsschrauben von medial und lateral in den Talus eingebracht (Abb.65/66).

Seither ist die Patientin in Ruhe und bei Belastung schmerzfrei und mit ihrem Zustand sehr zufrieden. 


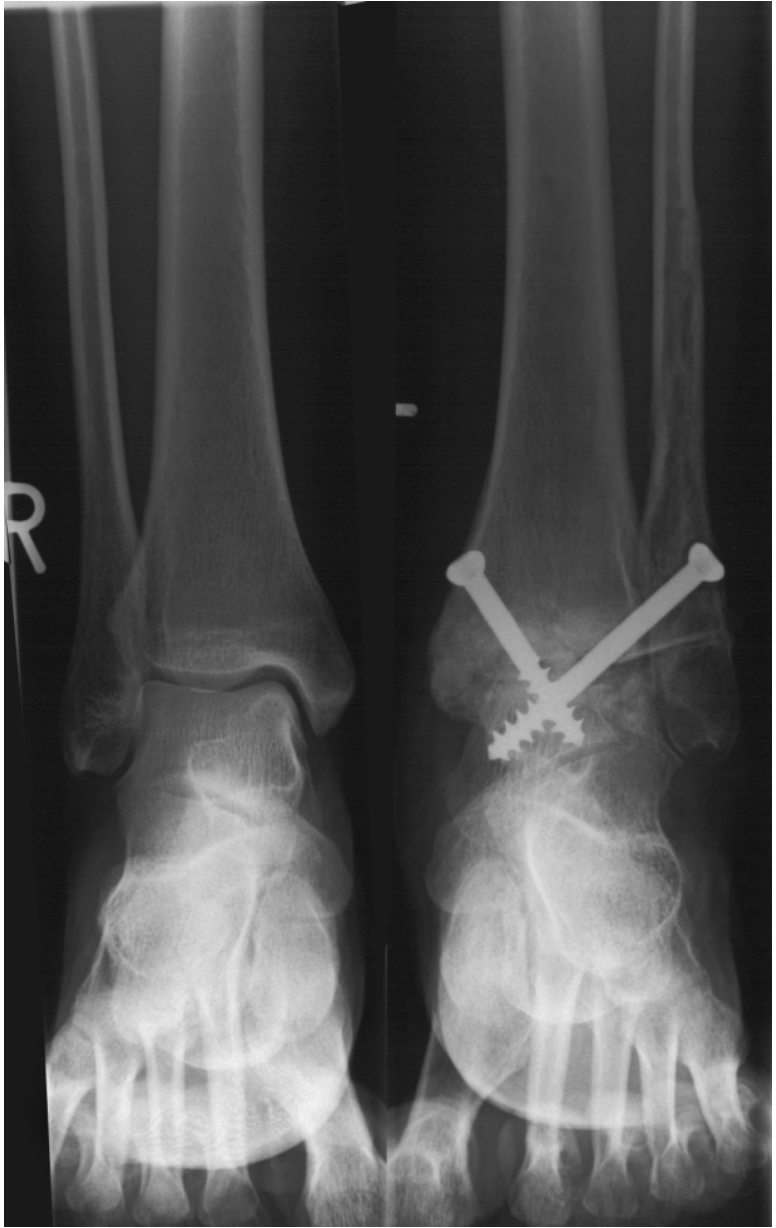

Abb.65: Z.n. KDA links mit 2

Kompressionsschrauben

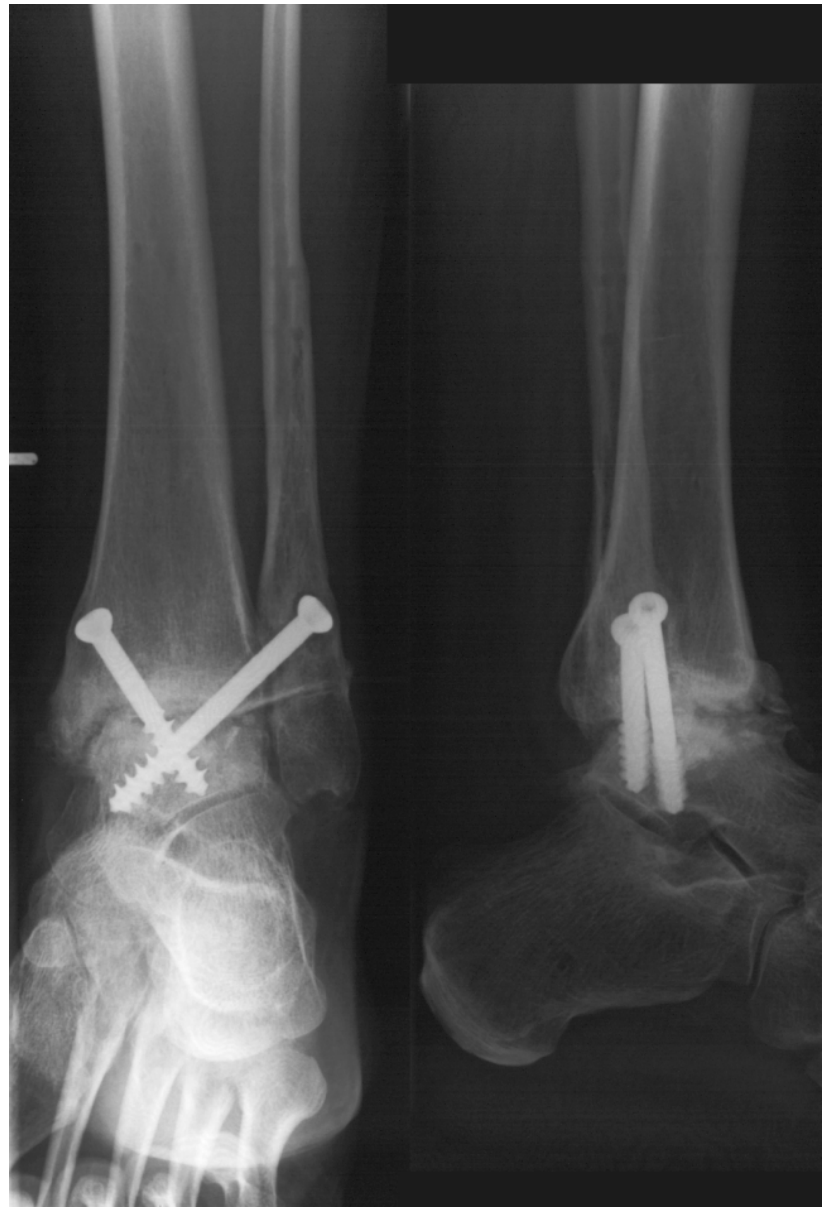

Abb.66: Z.n. KDA links in 2 Ebenen mit 2 Kompressionsschrauben 


\section{Abkürzungsverzeichnis}

a.-p.: anterior-posterior

Art:: Articulatio

Artt.: Articulationes

CT: Computertomographie

KDA: Knochen-Dübel-Arthrodese

Lig.: Ligamentum

Ligg.: Ligamenta

Lt.: laut

MRT: Magnetresonanztomographie

OSG: oberes Sprunggelenk

Proc.: Processus

USG: untere(s) Sprunggelenk(e)

Z.n.: Zustand nach 


\section{Literaturverzeichnis}

Ahlberg, A,A Henricson (1981): Late results of ankle fusion. Acta Orthop Scand $\underline{52},(1) 103-5$

Albert, E (1879): Zur Resektion des Kniegelenkes. Wien Med Press 20, 705708

Anderson, T, L Linder, U Rydholm, F Montgomery, J Besjakov,A Carlsson (2005): Tibio-talocalcaneal arthrodesis as a primary procedure using a retrograde intramedullary nail: a retrospective study of 26 patients with rheumatoid arthritis. Acta Orthop $\underline{76}$, (4) 580-7

Baciu, C,E Filibiu (1979): Rapid arthrodesis of the ankle joint via verticalisation of the joint space. Arch Orthop Trauma Surg $\underline{93}$, (4) 261-4

Bardenheuer, M, U Obertacke,C Waydhas (2000): Epidemiologie des Schwerverletzten. Unfallchirurg 103, 355-363

Bhan, S,R Malhotra (1998): Subtalar Arthrodesis for flexible hindfoot deformities in childreen. Arch Orthop Trauma Surg 117, 312-315

Boack, DH (2005): Arthroskopische in- situ -Arthrodese des Subtalar- Gelenkes. Orthopäde 24, (12) 1245-1254

Böhler, L (1931): Diagnosis, Pathology and treatment of fractures of the os calcis. J Bone Joint Surg Am 13, 75-89

Boldin, C, W Grechenig,J May (2004): Die operative Behandlung der Maisonneuve -Verletzung. Oper Orthop Traumatol 16, (4) 433-450

Bonnaire, F, R Cyffka, T Lein,R Hellmund (2001): Talusfrakturen. Trauma Beruf 3(Suppl 2), 192-200

Boobbyer, G (1981): The long-term results of ankle arthrodesis. Acta Orthop Scand $\underline{52}$, (1) 107-10

Buchner, M,D Sabo (2003 a): Ankle fusion attributable to posttraumatic arthrosis: a long-term followup of 48 patients. Clin Orthop Relat Res (406) 155-64

Buchner, M,D Sabo (2003 b): External or internal fixation for arthrodesis of the ankle -- a comparative study of perioperative and long-term results. Unfallchirurg 106(6), 472-7 
Buck, P, B Morrey,E Chao (1987): The optimum position of arthrodesis of the ankle. A gait study of the knee and ankle. J Bone Joint Surg Am $\underline{69}$, (7) 1052-62

Charnley, J (1951): Compression arthrodesis of the ankle and shoulder. J Bone Joint Surg Br $\underline{33 B}, 180-191$

Chen, Y, T Huang, H Shih, K Hsu,R Hsu (1996): Ankle arthrodesis with cross screw fixation. Good results in 36/40 cases followed 3-7 years. Acta Orthop Scand $\underline{67}$, (5) 473-8

Coester, L, C Satzmann, J Leopold,W Pontarelli (2001): Long term results following ankle arthrodesis for post traumatic arthritis. J Bone Joint Surg Am 83, 218-228

Cooper, P (2001): Complications of ankle and tibiotalocalcaneal arthrodesis. Clin Orthop Relat Res (391), 33-44

Debrunner, HU,WR Hepp: Orthopädisches Diagnostikum. 6. überarb. und erw. Aufl. Georg Thieme Verlag, Stuttgart, New York 1994, 203-222

Dennis, D, M Clayton, D Wong, R Mack,M Susman (1990): Internal fixation compression arthrodesis of the ankle. Clin Orthop Relat Res (253) 21220

Dresing, K,K Stürmer (2000): Press-fit bone dowel arthrodesis of the ankle or the subtalar joint using a diamond bone cutting system. Surgical technique and initial results in 10 patients. Unfallchirurg 103(8), 645-55

Eberl, R, A Kaminski,G Muhr (2006): Offene Talusfraktur. Trauma Beruf $\underline{8}$, 265271

Egol, K, M Amirtatharajah,N Tejwani (2004): Ankle stress test for predicting the need for surgical fixation of isolated fibular fractures. J Bone Joint Surg Am 86, 2393-2398

Essex-Lopresti, P (1993): The mechanism, reduction technique and results in fractures of the os calcis. Clin Orthop Relat Res $\underline{290}$, 3-16

Fellmann, J,H Zollinger (1996): Versteifungseingriffe am unteren Sprunggelenkwechselde Konzepte im Wandel der Zeit. Z Orthop Ihre Grenzgeb 134, 341-345

Fink, B (2005): Arthrodesen des unteren Sprunggelenks. Trauma Beruf $\underline{7},(1)$ 585-589

Fluckiger, G,M Weber (2005): The transfibular approach for ankle arthrodesis. Oper Orthop Traumatol 17(4-5), 361-79 
Frey, C, N Halikus, T Vu-Rose,E Ebramzadeh (1994): A review of ankle arthrodesis: predisposing factors to nonunion. Foot Ankle Int $\underline{15}$, (11) 581-4

Goebel, M, T Muckley, L Gerdesmeyer, M Militz,V Buhren (2003): Intramedullary nailing in tibiotalocalcaneal arthrodesis. Unfallchirurg 106(8), 633-41

Grass, R,H Zwipp (1998): Die Arthrodese des oberen Sprunggelenkes in der vier- Schrauben-Technik. Oper Orthop Traumatol 10, (2) 134-142

Grass, R, K Herzmann, A Biewener,H Zwipp (2000): Verletzungen der unteren tibiofibularen Syndesmose. Unfallchirurg 103, 520-532

Grass, R, A Blewener, S Rammelt,H Zwipp (2003): Frakturen des oberen Sprunggelenks-Klassifikation und operative Standardversorgung. Trauma Beruf 5 (Suppl 2), 272-276

Greisberg, J, M Assal, G Flueckiger,SJ Hansen (2004): Takedown of ankle fusion and conversion to total ankle replacement. Clin Orthop Relat Res (424) $80-8$

Gruen, G,D Mears (1991): Arthrodesis of the ankle and subtalar joints. Clin Orthop Relat Res (268), 15-20

Günter, U, P Jentsch,G Heller (2002): Anterograde intramedullary tibio-talo calcaneus arthrodesis (alMTCA) with spongiosaplasty in pseudarthrosis. Unfallchirurg 105(5), 474-7

Hagen, R (1986): Ankle arthrodesis. Problems and pitfalls. Clin Orthop Relat Res (202) 152-62

Helm, R (1990): The results of ankle arthrodesis. J Bone Joint Surg Br $\underline{72(1)}$, $141-3$

Hildebrand, K, R Buckley,N Mohtadi (1996): Functional outcome measures after displaced intra- articular calcaneal fractures. J Bone Joint Surg $\mathrm{Br} \underline{78}$, 119-123

Holt, E, S Hansen, K Mayo,B Sangeorzan (1991): Ankle arthrodesis using internal screw fixation. Clin Orthop Relat Res (268) 21-8

Holz, U (2006): Posttraumatische Arthrose: Arthrodese/Endoprothese. Trauma Beruf $\underline{8}, 20-25$

Hopgood, P, R Kumar,P Wood (2006): Ankle arthrodesis for failed total ankle replacement. J Bone Joint Surg Br $\underline{88}$, (8) 1032-8 
Horn, M (2003): Arthrodesen der Sprunggelenke. Trauma Beruf $\underline{5}$, 179-185

Jerosch, J (2003): Arthroskopisch assistierte Arthrodese AAA des oberen Sprunggelenkes. FussSprungg $\underline{1}$, 66-75

Jerosch, J,O Vollmert (2005): Arthrodeseverfahren am oberen Sprunggelenk. Trauma Beruf $\underline{7}, 78-84$

Jerosch, J, J Steinbeck, M Schroder,R Reer (1996): Arthroscopically assisted arthrodesis of the ankle joint. Arch Orthop Trauma Surg 115(3-4), 182-9

Jerosch, J, H Fayaz,H Senyurt (2006): Ankle arthrodesis versus ankle replacement: a comparison. Orthopäde 35(5), 495-505

Katcherian, D (1998): Treatment of ankle arthrosis. Clin Orthop Relat Res (349), 48-57

Kennedy, J, J Harty, K Casey, W Jan,W Quinlan (2003): Outcome after single technique ankle arthrodesis in patients with rheumatoid arthritis. Clin Orthop Relat Res (412), 131-8

Kitaoka, H (1991): Salvage of nonunion following ankle arthrodesis for failed total ankle arthroplasty. Clin Orthop Relat Res (268) 37-43

Kitaoka, H,G Patzer (1996): Clinical results of the mayo total ankle arthroplasty. J Bone Joint Surg Am 모, 1658-1664

Kitaoka, H, I Alexander, R Adelaar,J Nunley (1994): Clinical rating system for the ankle- hindfoot, midfoot, hallux and lesser toes. Foot Ankle $\underline{15}$, 349353

Klaue, K,D Bursic (2005): [The dorsolateral approach to the ankle for arthrodesis]. Oper Orthop Traumatol 17(4-5), 380-91

Koebke, J (2004): Klinische Anatomie und Biomechanik des Sprunggelenkes unter besonderer Berücksichtigung der Endoprothetik. FussSprungg 2, 2-6

Lance, E, A Paval, I Fries, I Larsen,RJ Patterson (1979): Arthrodesis of the ankle joint. A follow-up study. Clin Orthop Relat Res (142), 146-58

Lauge-Hansen, N (1950): Fractues of the ankle 2: Combined experimental/surgical and experimental roentgenologic investigation. Arch Surg $\underline{60}, 957-985$

Lauge-Pedersen, H (2003): Percutaneous arthrodesis. Acta Orthop Scand Suppl $\underline{74(307)}$, I, 1-30 
Mann, R, J Van Manen, K Wapner,J Martin (1991): Ankle fusion. Clin Orthop Relat Res (268) 49-55

Marcus, R, G Balourdas,K Heiple (1983): Ankle arthrodesis by chevron fusion with internal fixation and bone grafting. J Bone Joint Surg Am $\underline{65}$, 833838

Mazur, J, E Schwartz,S Simon (1979): Ankle arthrodesis. Long-term follow-up with gait analysis. J Bone Joint Surg Am $\underline{61}$, (7) 964-75

McConnell, T, W Creevy,P Tornetta (2004): Stress examination of supination external rotation- type fibular fractures. J Bone Joint Surg Am $\underline{86}$, 21712178

McGuire, M, R Kyle, R Gustilo,R Premer (1988): Comparative analysis of ankle arthroplasty versus ankle arthrodesis. Clin Orthop Relat Res (226), 17481

McRae, R: Klinisch Orthopädische Untersuchung 3. Aufl. Gustav Fischer Verlag Stuttgart, Jena, New York 1995, 213-260

Mittelmeier, H,M Nizard (1981): Technique and results of arthrodesis of the ankle joint with autocompression plates (author's transl). Z Orthop Ihre Grenzgeb 119(4), 418-21

Mittelmeier, H, W Hort,K Diehl (1975): Internal compression arthrodesis of the ankle joint (with examination of stability for comparison. Arch Orthop Unfallchir 81(1), 57-64

Moeckel, B, B Patterson, A Inglis, T Sculco (1991): Ankle arthrodesis. A comparison of internal and external fixation. Clin Orthop Relat Res (268), 78-83

Morgan, C, J Henke, R Bailey,H Kaufer (1985): Long-term results of tibiotalar arthrodesis. J Bone Joint Surg Am $\underline{67}$, (4) 546-50

Morrey, B,GJ Wiedeman (1980): Complications and long-term results of ankle arthrodeses following trauma. J Bone Joint Surg Am 62, (5) 777-84

Muckley, T, T Schutz, S Srivastava, M Goebel, O Gonschorek, V Buhren (2003): Ankle arthrodesis with intramedullary compression nailing. Unfallchirurg 106(9), 732-40

Muhle, C, L Frank,T Rand (1998): Tibiofibular Syndesmosis: High-Resolution MRI Using a Local Gradient Coil. J Comput Assist Tomogr 22, (6) 938944 
Müller, M, S Nazarian,P Koch (1987): Classification AO des fractures. Springer, Berlin, Heidelberg, New York

Müller, E, M Wick, G Muhr (1999): Surgical management of posttraumatic malalignments and arthroses in the ankle. Orthopade 28(6), 529-37

Müller-Gerbl, M (2001): Anatomie und Biomechanik des oberen Sprunggelenkes. Orthopäde $\underline{30}, 3-11$

Myerson, M,G Quill (1991): Ankle arthrodesis. A comparison of an arthroscopic and an open method of treatment. Clin Orthop Relat Res (268), 84-95

Nieny, K (1905): Zur Behandlung der Fußdeformitäten bei ausgedehnten Lähmungen. Arch Orthop Trauma Surg $\underline{3}$, (1)

Pankovich, A (1978): Fractures of the fibula proximal to the distal tibiofibular syndesmosis. J Bone Joint Surg Am 무, 335-341

Papa, J,M Myerson (1992): Pantalar and tibiotalocalcaneal arthrodesis for posttraumatic osteoarthrosis of the ankle and hindfoot. J Bone Joint Surg Am $\underline{74},(7)$ 1042-9

Paul, D, B Eckhardt, K Kühnel,H Schumann (1968): Erfahrungen mit der konservativen Behandlung der Malleolarfraktur. Arch Orthop Trauma Surg $\underline{63}, 273-287$

Phillips, W, H Schwartz,C Keller (1985): A prospective, randomizes study of the management of severe ankle fractures. J Bone Joint Surg Am 67, 67-78

Pisani, G: Fußchirurgie. Georg Thieme Verlag, Stuttgart, New York 1998, 2-96, 127-231

Platzer, W: Taschenatlas der Anatomie, Bewegungsapparat 8.Aufl. Georg Thieme Verlag, Stuttgart 2003, 222-225.

Rammelt, S, R Grass, A Biewener,H Zwipp (2003): Bandverletzung am oberen Sprunggelenk. Trauma Beruf $\underline{5}, 170-178$

Rammelt, S, R Grass, A Biewener,H Zwipp (2004): Anatomie, Biomechanik und Klassifikation der Sprunggelenkfrakturen. Trauma Berufskrankh $\underline{6(4)}$, (4) 384-392

Rammelt, S, R Grass,H Zwipp (2007): Frakturen des oberen Sprunggelenks. FussSprungg $\underline{5}$, (2) 88-103

Rammelt, S, R Grass,H Zwipp (2008): Sprunggelenkfrakturen. Unfallchirurg 111, 421-438 
Rehart, S, A Peters,F Kerschbaumer (1999): Arthrodesen am unteren Sprunggelenk beim Erwachsenen. Orthopäde 28, 770-777

Reiser, M, F Kuhn,J Debus: Radiologie. Georg Thieme Verlag, Stuttgart 2006

Richter, J, W Schulze,G Muhr (1999): Stabile Knöchelbrüche. Indikation zur Operation oder konservativen Therapie? Orthopäde 28, (6) 493-499

Rowan, R,K Davey (1999): Ankle arthrodesis using an anterior AO T plate. J Bone Joint Surg Br $\underline{81}$, (1) 113-6

Rüedi, T, R Buckley,C Moran: AO Principles of Fractures Managements. Georg Thieme Verlag, Stuttgart, New York 2007

Russotti, G, J Cass,K Johnson (1988): Isolated talocalcaneal arthrodesis. A technique using moldable bone graft. J Bone Joint Surg Am $\underline{70}$, 14721478

Sanders, R (1992): intra-articular fractures of the calcaneus: present state of the art. J Orthop Trauma $\underline{6}$, (2) 252-265

Sanders, R (2000): Displaced íntra-articular fractures of the calcaneus. J Bone Joint Surg Am 82, 225-250

Schaap, E, J Huy,A Tonino (1990): Long-term results of arthrodesis of the ankle. Int Orthop 14, (1) 9-12

Schuh, H,M Hausel (2000): Die Schwierigkeiten in der Beurteilungen von Nachuntersuchungsergebnissen bei plattenosteosynthetisch versorgter Kalkaneusfraktur. Unfallchirurg 103, (4) 295-300

Schulze, W, J Richter, O Russe, P Ingelfinger,G Muhr (2002): Surgical treatment of talus fractures: a retrospective study of 80 cases followed for 1-15 years. Acta Orthop Scand $\underline{73}$, (3) 344-51

Scranton, PJ (1985): Use of internal compression in arthrodesis of the ankle. J Bone Joint Surg Am $\underline{67}$, (4) 550-5

Scranton, PJ (1991): An overview of ankle arthrodesis. Clin Orthop Relat Res (268), 96-101

Scranton, PJ, F Fu,T Brown (1980): Ankle arthrodesis: a comparative clinical and biomechanical evaluation. Clin Orthop Relat Res (151), 234-43

Seiler, H (1999): Das obere Sprunggelenk. Biomechanik und funktionelle Anatomie. Orthopäde $\underline{28}, 460-468$

Stuhler, TH: Arthrodesen. Georg Thieme Verlag, Stuttgart 1994, 229-236 
Thermann, H, T Hufner, H Schratt, C Held,H Tscherne (1999): Subtalar fusion after conservative or surgical treatment of calcaneus fracture. A comparison of long-term results. Unfallchirurg 102(1), 13-22

Thordarson, D (1993): Ankle fusion with internal fixation. Techniques in Orthop 8, (1) $44-49$

Thordarson, D, K Markolf,Ar Cracchiolo (1992): Stability of an ankle arthrodesis fixed by cancellous-bone screws compared with that fixed by an external fixator. A biomechanical study. J Bone Joint Surg Am 74(7), 1050-5

Thordarson, D, S Motamed, T Hedman, E Ebramzadeh,S Bakshian (1997): The effect of fibular malreduction on contact pressures in an ankle fracture malunion model. J Bone Joint Surg Am $\underline{79}$, 1809-1815

Trieb, K, D Wirtz, H Durr,D Konig (2005): Results of arthrodesis of the upper ankle joint. Z Orthop Ihre Grenzgeb 143(2), 222-6

Trnka, H, M Easley, P Lam, C Anderson, L Schon,M Myerson (2001): Subtalar distraction bone block arthrodesis. J Bone Joint Surg Br $\underline{83}$, (6) 849-54

Vienne, P (2005): Interposition arthrodesis of the ankle. Oper Orthop Traumatol $\underline{17(4-5)}, 502-17$

Weber, B: Verletzungen des oberen Sprunggelenkes. Aktuelle Probleme der Chirurgie, Bd 3. Huber, Bern, Stuttgart 1996

Wenzl, M, S Fuchs, C Jürgens,D Wolter (2001): Kalkaneusfrakturen. Trauma Beruf $\underline{3}$, (2) $223-226$

Wülker, N, C Flamme, A Müller,C Wirth (1997): 10 years follow-up of athrodeses of the hindfoot joints and upper ankle joint. Z Orthop Ihre Grenzgeb 135(6), 509-15

Xenos, J, W Hopkinso, M Mulligan,E Olson (1995): The tibiofibular syndesmosis. Evaluation of the ligamentous structures. Method of fixation, and radiographic assessment. J Bone Joint Surg Am $\underline{77}$, 847856

Zwipp, H: Chirurgie des Fußes. Springer, Wien, New York 1994

Zwipp, H,R Grass (2005): Ankle arthrodesis after failed joint replacement. Oper Orthop Traumatol 17(4-5), 518-33

Zwipp, H, R Grass, S Rammelt,C Dahlen (1999): Arthrodesis - non-union of the ankle. Arthrodesis failed. Chirurg $\underline{70(11)}, 1216-24$ 
Zwipp, H, S Rammelt,S Barthel (2005): Kalkaneusfraktur. Unfallchirurg $\underline{108}$, 737-748 


\section{Anhang}

\subsection{Traumatologische Klassifikationen}

\subsubsection{Klassifikation nach Lauge-Hansen}

1950 wurde von Lauge-Hansen (1950) durch experimentelle Untersuchungen bzw. detaillierte klinische Beobachtung ein Zusammenhang zwischen UnfallPathomechanismus und Frakturmorphologie erkannt.

Auf diesen Ergebnissen beruht die heute noch existente Lauge-HansenKlassifikation.

Mit dieser ist es möglich, die Indikation für eine adäquate Therapie zu stellen. Die Entscheidung beruht auf der Kenntnis von Frakturmorphologie und Stabilität.

Grundlage, mit der der dänische Chirurg Lauge-Hansen seine unfallabhängige Klassifikation erstellte, ist zum Einen die Kenntnis der Stellung des Fußes kurz vor bzw. zu Beginn des Unfallmechanismus und zum Anderen die Art bzw. Richtung der Krafteinwirkung während des Unfalles.

Wichtig ist bei dieser Klassifikation, dass Lauge-Hansen mit Eversion die Außenrotation des Talus bzw. des Fußes in der Malleolengabel meint (Rammelt et al. 2008). Im englischen Sprachraum wurde dafür der Begriff „external rotation" geprägt (Pankovich 1978).

Die Stellung des Fußes vor dem Unfall kann grob gesagt in Pro- und Supination unterteilt werden.

Wenn der Fuß in Pronationsstellung ist, beginnt die Zerstörung am Innenknöchel. Anders verhält es sich bei supiniertem Fuß, wo der Außenknöchel zuerst zu Schaden kommt (Rammelt et al. 2008).

Wenn sich der Fuß in Pronationsstellung befindet, ergibt sich automatisch eine Dorsalextension. Das führt dazu, dass sich der breite Teil der Trochlea tali in 
der Malleolengabel einstellt. Daraus ergibt sich eine gewisse Vorspannung der Syndesmose.(Rammelt et al. 2008)

In Dresden wurden von 1958 - 1965364 Malleolarfrakturen behandelt und nachuntersucht. Die Behandlung erfolgte "streng konservativ". Nach einer Reposition in Allgemeinanästhesie und dem Anlegen eines ungepolsterten und gespaltenen Gipses, wurde das Bein bereits in der 2. Woche in einem Unterschenkelgehgips belastet. In der nachfolgenden Analyse wurden die Frakturen nach Lauge-Hansen klassifiziert. $61,1 \%$ der Frakturen waren Supinations-Eversions-Frakturen.

15,8\% nahmen die Pronations-Abduktions-Frakturen und 15,9\% die PronationsEversions-Frakuren ein. Nur 7,3\% waren Supinations-Adduktions-Frakturen (Paul et al. 1968). So ergeben sich in dieser Klassifikation vier verschiedene Frakturtypen, auf die im Folgenden genauer eingegangen wird.

\subsubsection{Supinations-Eversions-Fraktur}

Bei der Supinations-Eversions Fraktur befindet sich der Fuß in Supinationsstellung. Diese ist mit ca. 2/3 die häufigste Frakturform (Rammelt et al. 2008).

Hierbei kommt es zu einer forcierten Torsion des Talus nach außen durch eine axiale Krafteinwirkung. Bedingt wird dies durch eine Außentorsion des Unterschenkels.

In diesem supinierten Zustand befindet sich die Trochlea in einer zur Malleolengabel quer eingestellten Position.

Im Stadium 1 kommt es zu einer interligamentären Ruptur des Lig. tibiofibulare anterius mit oder ohne knöchernem Ausriss. Ein knöcherner Ausriss der vorderen Syndesmose an der Tibia wird als Tubercule de Chaput bezeichnet. Wenn die Syndesmose an der Fibula knöchern ausreißt, wird dies WagstaffFragment genannt. Ein knöcherner Ausriss der hinteren Syndesmose an der Tibia geht mit einem Ausriss des Tuberculum tibiae posterior (fälschlicher Weise Vollkmann'sches Dreieck genannt) einher (Zwipp 1994; Rammelt et al. 2008). 
Es muss festgehalten werden, dass eine singuläre Ruptur des Lig. tibiofibulare anterius noch keine mechanisch bedeutsame Instabilität der Malleolengabel ausmacht (Xenos et al. 1995; Grass et al. 2000; Rammelt et al. 2008).

Eine pathologische Beweglichkeit der Knochengabel entsteht erst, wenn zusätzlich das Lig. tibiofibulare interosseum und/oder das Lig. tibiofibulare posterius oder die Membrana interossea verletzt sind (Xenos et al. 1995).

In dem Stadium 2 kommt es zu einer schrägen, spiralförmigen Fraktur des Außenknöchels auf Höhe der Syndesmose. Bedingt wird dies durch eine zunehmende Eversion des Talus. Hierbei bleiben das Lig. tibiofibulare interosseum und die Membrana interossea unbeschädigt.

Durch zunehmende Außentorsion des Talus kann es im 3. Stadium zu einem Ausriss des Volkmann-Dreiecks (Tuberculum posterius tibiae) oder zu einer Ruptur des Lig. tibiofibulare posterius kommen.

Im Stadium 4 frakturiert zusätzlich der Innenknöchel unter Umständen mit einer zusätzlichen Deltabandruptur (Grass et al. 2003; Rammelt et al. 2008).

\subsubsection{Pronations-Abduktions-Fraktur}

Die Pronations-Abduktions-Fraktur ist eine instabile Situation. Ein sich in Pronations- und Abduktionsstellung befindlicher Fuß wird durch axiale Kräfte belastet.

Hierbei kommt es im ersten Stadium zu einem Riss des Lig. collaterale mediale und eventuell zu einem zusätzlichen knöchernen Ausriss des Malleolus medialis.

Im Stadium 2 reißt das Lig. tibiofibulare anterius und/oder posterius eventuell knöchern aus.

Es kann zu einer Fraktur des Volkmann-Dreiecks kommen.

Wenn die Fibula auf Höhe der Syndesmose mehrfragmentär gebrochen ist, wird von einem Stadium 3 gesprochen. Das Lig. tibiofibulare interosseum ist dabei nur selten verletzt (Grass et al. 2003; Rammelt et al. 2008). 


\subsubsection{Pronations-Eversions-Fraktur}

Beim Pronations-Eversions-Trauma steht der Fuß unter axialer Belastung in Pronationstellung und ist dabei evertiert. Zusätzlich ist eine Innentorsion des Unterschenkels vorhanden, die den Talus in der Malleolengabel quer einstellt. Durch diesen Mechanismus kommt es im ersten Stadium meist zu einer Fraktur des Innenknöchels mit potentieller Ruptur des Deltabandes. Da der Talus keine mediale Führung mehr hat, kann er sich weiter nach außen drehen und sich unter der Tibia valgisch einstellen.

Im zweiten Stadium kommt es zu einer weiteren Fehlstellung des Talus bedingt durch eine vollkommene Sprengung der medialen ligamentären Führung. So sind jetzt die Ligg. tibiofibulare anterius, posterius und inerosseum sowie die Membrana interossea gerissen. Der Talus kann auf diese Weise seine Valgusfehlstellung genauso wie seine Außenrotationsbewegung fortsetzen.

Aufgrund dieser Talusfehlstellung kann es, wenn die einwirkende Kraft anhält, zu einer hohen suprasyndesmalen Spiralfraktur der Fibula kommen. Dabei wird die Membrana interossea bis auf die Höhe der Fibulafraktur komplett zerstört. Dies wird dann als Stadium 4 bezeichnet (Grass et al. 2003; Rammelt et al. 2008).

Eine besondere Form dieses Frakturtypes stellt die Maisonneuve-Fraktur da. Dabei handelt es sich um eine proximale Fibula Fraktur, die häufig subkapital verläuft und mit einer Sprengung der Malleolengabel einhergeht. Dabei reißt die Syndesmose und häufig die Membrana interossea. Es können Begleitverletzungen wie die Innenknöchelfraktur mit oder ohne Deltabandruptur oder eine Fraktur der hinteren Tibiakante vorkommen. Letzteres wird als hohe Typ-C-Weber Verletzung bezeichnet (Boldin et al. 2004; Rammelt et al. 2008). 


\subsubsection{Supinations-Adduktions-Fraktur}

Wenn eine axiale Kraft auf einen sich in Supinations- und Adduktionsstellung befindlichen Fuß einwirkt und diesem einen Schaden zufügt, wird von einer Supinations-Adduktions Fraktur gesprochen.

Im ersten Stadium kann ein Ausriss der Ligg. fibulotatare anterius und/oder posterius und/oder fibulocalcaneare diagnostiziert werden. Bei zusätzlichem Ausriss des lateralen Knöchels liegt eine Weber-A-Fraktur vor.

Wenn der Supinationsstress weiter anhält, kommt es im zweiten Stadium zu einer schrägen Fraktur des Innenknöchels oder einer Ruptur des Lig. deltoideum. Hiernach ist die Malleolengabel instabil (Grass et al. 2003; Rammelt et al. 2008). 


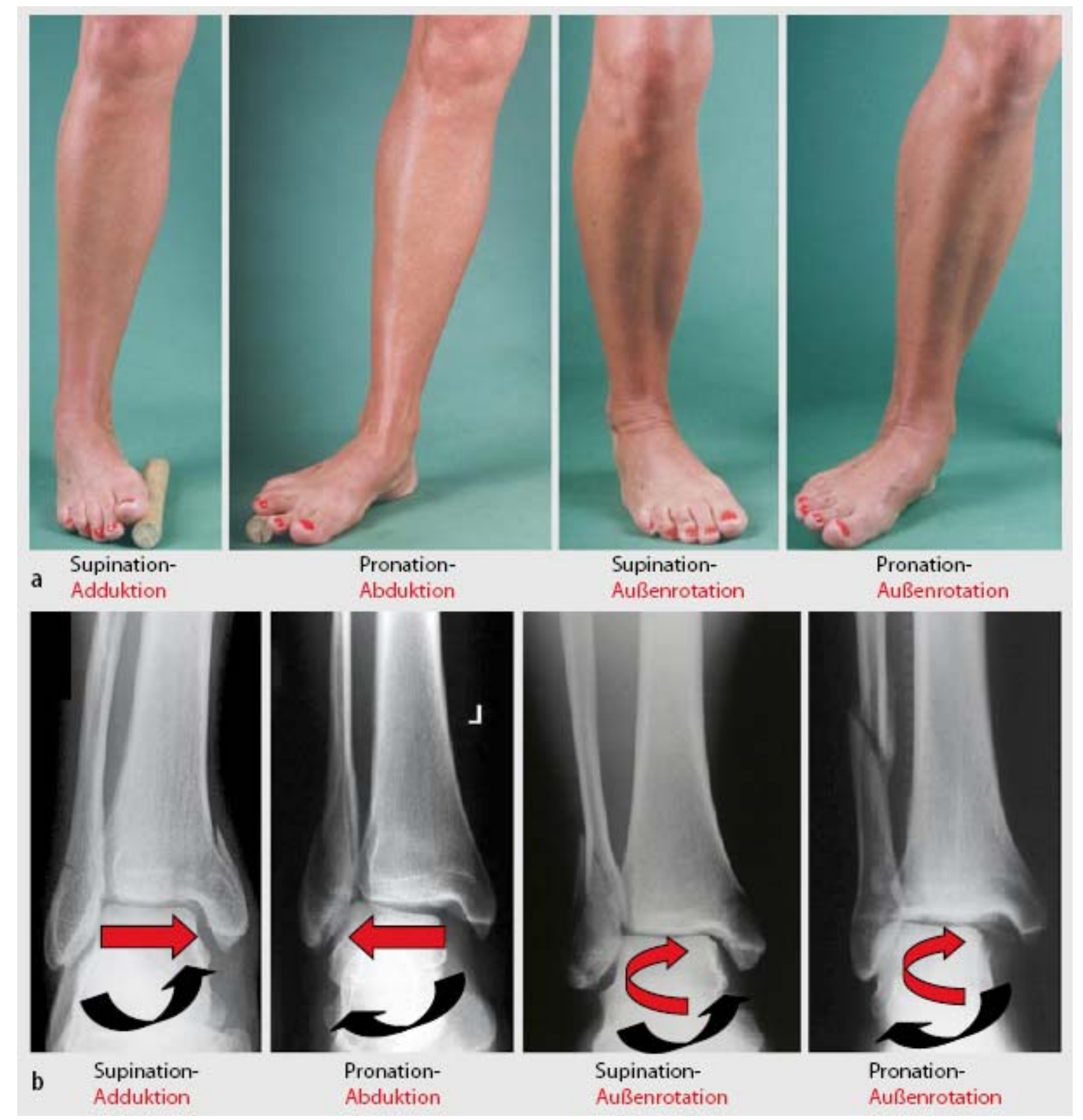

Abb.1 Entstehung der vier verschiedenen Frakturtypen nach Lauge-Hansen. Schwarz ist die Stellung des Fußes zum Unfallzeitpunkt. Rot ist die Richtung der Gewalteinwirkung (Rammelt et al. 2008, S.424). 


\subsubsection{Klassifikation nach Dennis Weber}

In dieser Klassifikation wird die Frakturhöhe der Fibula ausschließlich in Bezug zum Syndesmosenkomplex gesehen.

Typ-A ist eine infrasyndesmale, stabile Fibulafraktur. Bei dem Typ-B handelt es sich um eine transsyndesmale Fibulafraktur, bei der die Syndesmose nicht obligat verletzt und somit nicht immer instabil ist. Typ-C hingegen ist immer instabil, da dies eine suprasyndesmale Fibulafraktur mit obligat zerstörter Syndesmose darstellt (Weber 1966; Rammelt et al. 2008).

Bezüglich der Aussagen zur Stabilität und der Operationsindikation kann diese Klassifikation jedoch nur eingeschränkt verwendet werden, da die hierfür notwendige Kenntnis von dem Zustand der medialen Strukturen unberücksichtigt bleibt (Rammelt et al. 2008).

\begin{tabular}{|lll|}
\hline Frakturtyp & Höhe der Fibulafraktur & Syndesmosenstabilität \\
\hline A & Infrasyndesmal & Intakt \\
\hline B & Transsyndesmal & Fraglich intakt \\
\hline C & Suprasyndesmal & Nicht intakt \\
\hline
\end{tabular}

Abb.3 Klassifikation nach Weber (Rammelt et al. 2008, S.428) 


\subsubsection{AO-Klassifikation}

Die Benennung erfolgt anhand von 4 Kategorien:

1.Körperregion Bsp.: 6=Becken, $8=F u ß, 3=$ Oberschenkel

2.Position Bsp.: 1=proximal, 2=diaphysär, 3=distal

3.Kompliziertheit Bsp.: A=einfache Fraktur, $B=$ Fraktur mit partieller Gelenkbeteiligung oder Keilfraktur, $\mathrm{C}=$ komplexe Gelenkfraktur oder komplexe Fraktur

4.Schwere Bsp.: 1=leicht, 2=mittel, 3=schwer

(Müller M et al. 1987)

Beispiel: 33A1: einfache distale Femurschaftfraktur

So können zum Beispiel Weber-B-Frakturen in stabil und instabil unterteilt werden. B1-Verletzungen sind reine Außenknöchelfrakturen, während B2Verletzungen am Innenknöchel diagnostiziert werden und instabil sind (Müller $M$ et al. 1987). Auf diese Weise kann mit den Weber-A-Frakturen verfahren werden. Eine A1-Fraktur ist eine stabile Verletzung ohne Innenseiten-Schaden. Eine A2-Verletzung ist instabil, da der mediale Knöchel frakturiert ist. Die Frakturgenese kann in den Untergruppen nicht genau verfolgt werden. In der alltäglichen Klinik hat sich eine rein deskriptive Beschreibung durchgesetzt. Hiernach werden die Sprunggelenkfrakturen in uni-, bi- und trimalleoläre Formen eingeteilt.

Die kräftige hintere Tibiakante entspricht hierbei dem „dritten Knöchel“. Wenn die hintere Syndesmose knöchern ausreißt, ist dies das entsprechende Fragment. Dieses Fragment wurde 1828 von Earle beschrieben. In Deutschland wird dies fälschlicher Weise als "hinteres Volkmann Dreieck" bezeichnet, weil Volkmann ein anterolaterales Kantenfragment beschrieben hatte (Zwipp 1994; Rammelt et al. 2008). 


\subsubsection{Pathogenese der Typ-A-Fraktur}

Der Frakturmechanismus ist wie bei einem Supinations-Adduktions-Trauma.

Der Schaden tritt zuerst auf der lateralen Seite auf. So reißt hier meistens das Lig. talofibulare anterius zuerst. Bei weiterer Belastung führt dies zu einem osteoligamentären Ausriss oder direkt zu einer Frakturierung des lateralen Malleolus auf Höhe oder unterhalb des tibialen Gelenkplateaus.

Wenn die einwirkende Kraft weiter anhält, adduziert bzw. supiniert der Fuß weiter nach medial und führt zu einer Kompressionsfraktur des medialen Malleolus (Rüedi et al. 2007).
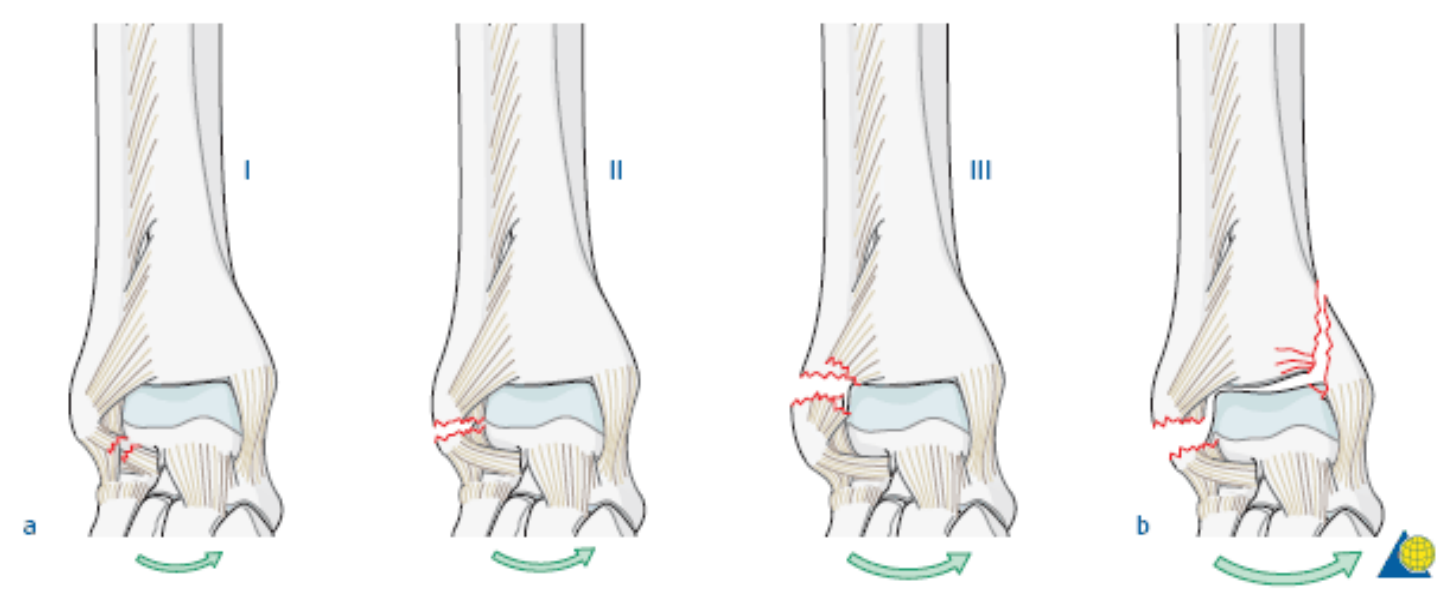

Abb.4 Pathogenese der Typ-A-Fraktur von links nach rechts ansteigend

a/1: Riss des Lig. tibiofibulare anterius

2: osteoligamentärer Ausriss

3: horizontale Fibulafraktur

b: durch Zunahme der Kraft: Kompressionsfraktur des Malleolus medialis

Abb.: AO-Foundation Homepage 


\subsubsection{Pathogenese der Typ-B-Fraktur}

Bei der Typ-B-Fraktur wirkt eine axiale Kraft auf den supinierten Fuß.

Dabei verläuft die Fraktur des lateralen Außenknöchels schräg auf Höhe der Syndesmose von anterior nach posterior. Die Begründung liegt in der schrägen Einstellung des Talus in der Malleolengabel und der Außenrotationsbewegung (Eversion) des Fußes. Ohne weitere Krafteinwirkung liegt eine stabile und nicht dislozierte Fraktur vor. Hält die einwirkende Kraft jedoch an, so verstärkt sich die Fehlstellung des Talus und die Fibulafraktur wird disloziert.

Bei weiterer Rotation des Talus frakturiert zusätzlich das Volkmann'sche Dreieck und des Weiteren können das Lig. deltoideum bzw. der Malleolus medialis verletzt werden (Rüedi et al. 2007).
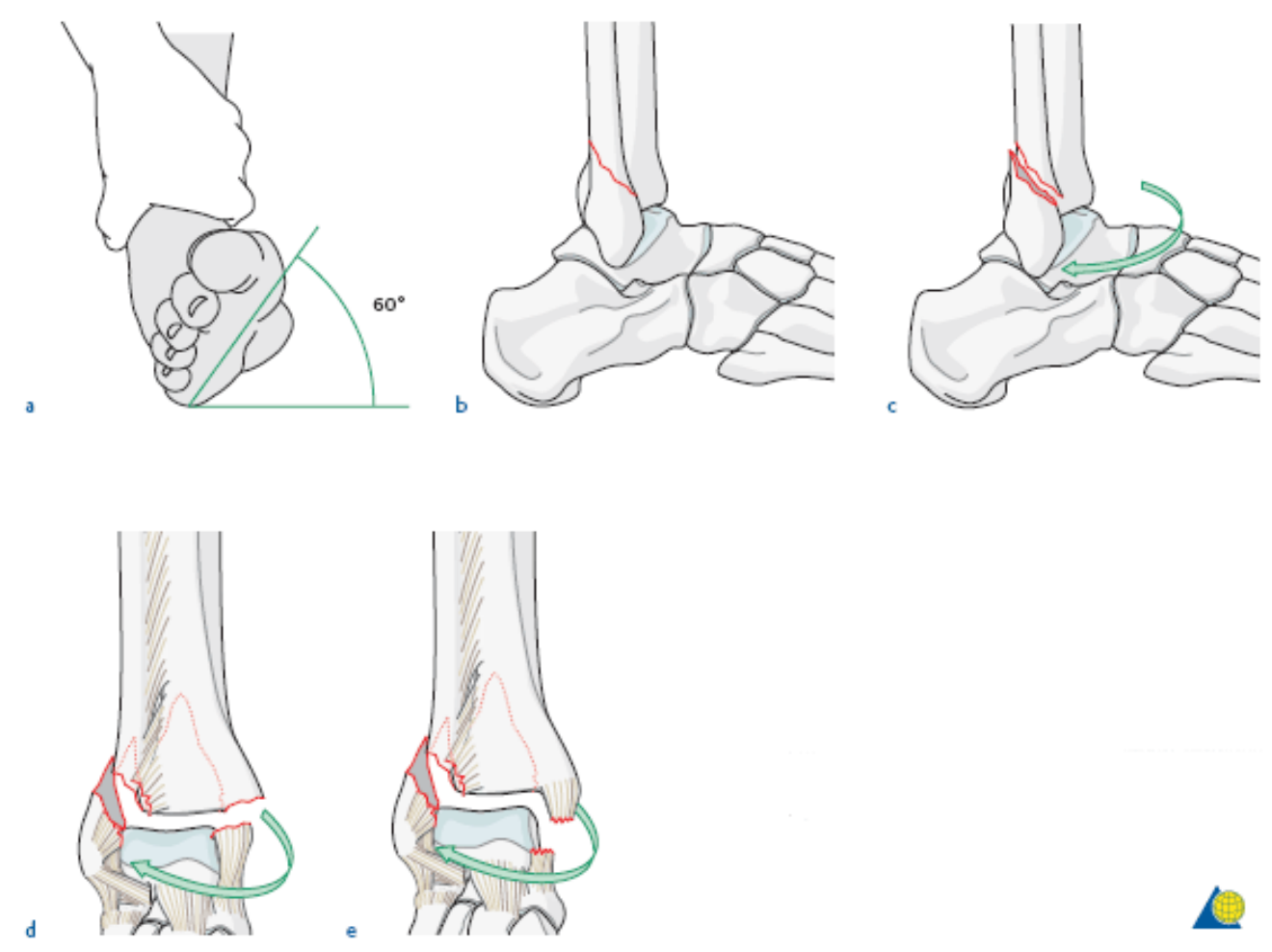

Abb.5 Pathogenese der Typ-B-Verletzung

a: supiniert und adduzierter Fuß

b: schräge Fraktur der Fibula

c: Dislokation der Fibulafraktur

d-e: Verletzung des Volkmann'schen Dreieck, Lig. deltoideum und Malleolus medialis

Abb.: AO-Foundation Homepage 


\subsubsection{Pathogenese der Typ-C-Fraktur}

Die Typ-C-Fraktur ist eine Form der Pronations-Eversions-Fraktur.

Hierbei ist der Fuß in Pronationsstellung und die medialen Bänder stehen unter Spannung. Darauf folgt durch die einwirkende Kraft eine Außenrotation des Fußes.

Der erste Schaden tritt hierbei auf der medialen Seite auf. Meist kann ein partieller bis totaler Riss des Lig. deltoideum und/oder eine Fraktur des medialen Malleolus diagnostiziert werden. Die Außenrotation des Talus führt sekundär zu einer Ruptur des Lig. tibiofibulare anterius (anteriore Syndesmose) und als nächstes zu einem Riss der interossären Syndesmose. (Lig. tibiofibulare interosseum)

Bei weiterem Anhalten der einwirkenden Kraft führt der Rotationsstress zu einer Fraktur der Fibula. Die Höhe der Frakturierung hängt davon ab wie weit proximal die Membrana interossea rupturiert (Rüedi et al. 2007).
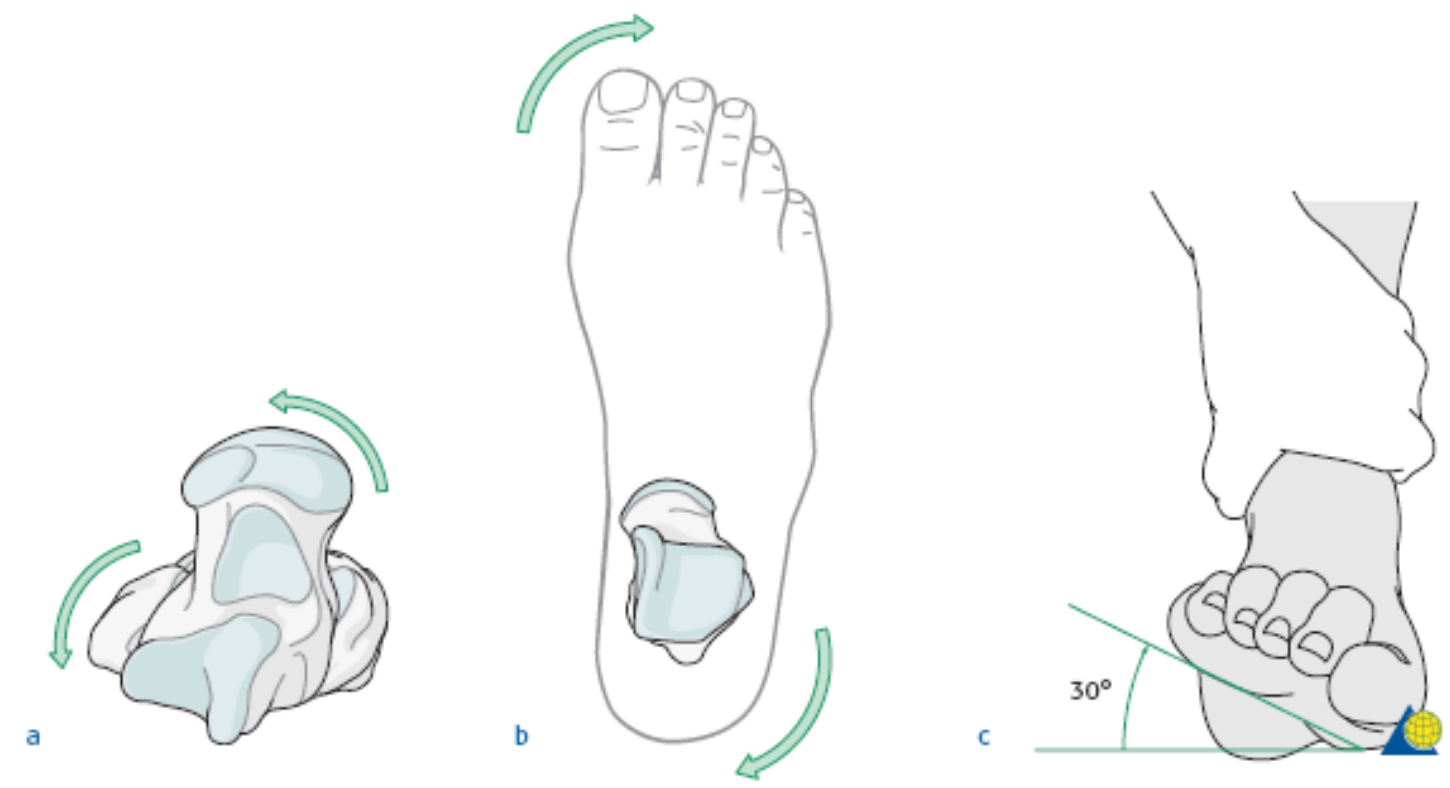

Abb. 6 Pathogenese der Typ-C-Fraktur a-c: der Fuß steht in Pronationsstellung, während er in Eversionsstress gerät. Abb.: AO-Foundation Homepage 


\subsubsection{Klassifikation und Pathogenese der Kalkaneusfrakturen}

Eine anatomische Klassifikation der Fersenbeinfraktur konnte Böhler (1931) schon 1931 einführen. Er unterschied 8 verschiedene Kalkaneusfraktur-Typen basierend auf anatomisch-pathologischen Beobachtungen.

Die Frakturen des Kalkaneus können in intraartikuläre und extraartikuläre Formen eingeteilt werden. Zu ersterer gehört die von Essex-Lopresti (1993) 1952 beschriebene klassische Einteilung, die sich weiter in ,joint depressiontype" und „tongue-type" unterteilen lässt. In aller Regel entsteht die klassische intraartikuläre Fersenbeinfraktur durch eine axiale Stauchung wie zum Beispiel der Sturz aus großer Höhe. Wenn eine intraartikuläre Fraktur vorliegt, wird der Processus lateralis tali wie ein Keil in den Kalkaneus eingetrieben. Daraufhin zerbricht der Kalkaneus in zwei Fragmente durch eine primäre Frakturlinie. Zum Einen in ein hinteres posterolaterales und in ein vorderes anteromediales (oder superior-mediales), das Sustentaculum tragende Stück (Zwipp et al. 2005; Reiser et al. 2006).

Wenn eine höhere Kraft einwirkt kommt es zu einer sekundären Frakturlinie. Dabei wird vom posteolateralen Fragment eine posteriore Facette abgetrennt. Daraufhin entsteht eine sekundäre Frakturlinie. Diese kann einerseits die subtalare Gelenkfläche umrundend nach kranial verlaufen und wird dann ,joint depression-type" genannt. Andererseits kann die sekundäre Frakturlinie horizontal zum Tuber calcanei verlaufen. Diese Frakturvariante wird als „tongue-type“ bezeichnet. Morphologisch resultiert daraus eine Höhenminderung und eine Verbreiterung des Kalkaneus (Reiser et al. 2006).

Des Weiteren kann die relativ dünne laterale Kalkaneuswand („lateral bulge“) ausbrechen und die Peronealsehnen inkarzerieren. Des Weiteren kann es zu einem Anstoß des äußeren Malleolus an den Kalkaneus kommen (Wenzl et al. 2001; Zwipp et al. 2005). 


\subsubsection{Klassifikation nach Sanders}

Sanders (1992) etablierte eine Klassifikation, die auf der Anzahl der Frakturlinien auf dem koronaren $\mathrm{CT}$ auf Höhe der posterioren Facette beruht. Dies ist im nordamerikanischen Raum eine bevorzugte Klassifikation zur praxisorientierten und therapiegerichteten Evaluation. Hier gilt:

Typ 1: alle nicht dislozierten Frakturen (unabhängig von der Zahl der Frakturlinien)

Typ 2: $\quad$ eine Frakturlinie in der posterioren Facette

Typ 3: $\quad$ zwei Frakturlinien in der posterioren Facette

Typ 4: drei oder mehr Frakturlinien in der posterioren Facette (Trümmerfraktur)

(Sanders 1992). 


\subsection{Anschreiben}

Sehr geehrte(r) Herr/Frau NN

Sie wurden im Rahmen einer Versteifungsoperation (Arthrodese) am Sprunggelenk im Universitätsklinikum Göttingen von uns behandelt.

Im Rahmen einer Forschungsarbeit untersuchen wir die Ergebnisse nach Arthrodesen am Sprunggelenk. In Göttingen wurden verschiedene Operationsverfahren für Versteifungsoperation am Sprunggelenk in den letzten Jahren durchgeführt.

Ziel der Arbeit ist es, aus den Ergebnissen Schlüsse für evtl. neue Operationsverfahren zu erhalten. Mit Ihren Angaben helfen Sie dieses Ziel zu erreichen.

Um dies tun zu können, benötigen wir einen möglichst genauen Eindruck über Ihr derzeitiges Befinden bzw. Ihren Zustand. Dazu finden Sie beiliegend einen Fragebogen, der nur wenige Minuten Ihrer Zeit in Anspruch nimmt.

Zusätzlich würden wir uns gern einen persönlichen Eindruck von Ihnen machen (dabei können wir Ihnen auch gern mit dem Fragebogen behilflich sein). Hierzu werden wir uns telefonisch bei innen melden, um mit Ihnen einen Termin zu vereinbaren. Die Teilnahme an der Studie insgesamt ist freiwillig. Risiken bestehen für Sie durch Teilnahme an der Studie nicht.

Wir möchten Sie freundlich bitten, den ausgefüllten Fragebogen bis zum NN. Monat 2010 an uns zurück zu senden. Ein frankierter Rückumschlag liegt bei.

Bitte beachten Sie: Selbstverständlich werden Ihre Daten im Rahmen der ärztlichen Schweigepflicht anonym und vertraulich behandelt. Ihre Daten werden vollständig anonymisiert, so dass man später nicht zurückverfolgen kann, von wem die Daten sind. Bis zur Komplettierung der Datensätze werden die Datensätze pseudonymisiert. Dies bedeutet, dass die Daten mit einer Verschlüsselung ohne Namensnennung nur mit Nummern versehen werden. Die Zuordnung der Daten ist nur möglich, wenn hierfür der Schlüssel eingesetzt wird, mit dem die Daten pseudonymisiert wurden. Die Personen bezogenen Daten werden unter besonderen Schutzvorkehrungen getrennt von den pseudonymisierten Daten aufbewahrt. Eine Entschlüsselung ist nur durch den verantwortlichen Studienarzt möglich. Dritte erhalten keinen Einblick in die Originalunterlagen.

Wir weisen Sie darauf hin, dass die Teilnahme vollkommen freiwillig ist, aber Sie helfen uns, mit Ihrer Mitarbeit die Behandlung zukünftiger Patienten zu verbessern. Sie können Ihre Teilnahme und das Einverständnis zur Datennutzung und -speicherung jederzeit ohne Angabe von Gründen widerrufen. 
Vielen Dank für ihre Mitarbeit

Mit freundlichen Grüssen

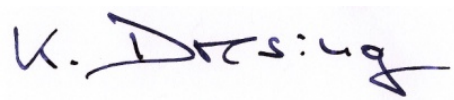

Prof. Dr. Klaus Dresing

Ltd. Oberarzt

Unfallchirurgie, Plastische und Wiederherstellungschirurgie

11.3 Patientenfragebogen

\section{Fragebogen zu Ihrer Arthrodese}


Dieser Fragebogen wird zur Auswertung pseudonymisiert!

\section{Zufriedenheit:}

O Ich bin mit meinem Zustand bzw. Operationsergebnis sehr zufrieden

$\mathrm{O}$ Ich bin mit meinem Zustand bzw. Operationsergebnis etwas zufrieden

$\mathrm{O}$ Ich bin mit meinem Zustand bzw. Operationsergebnis kaum zufrieden

O Ich bin mit meinem Zustand bzw. Operationsergebnis nicht zufrieden

Wenn Sie hierzu noch etwas anmerken

wollen:

\section{Schmerzen:}

O Ich habe keine Schmerzen

O Ich habe leichte, gelegentliche Schmerzen

O Ich habe erträgliche, tägliche Schmerzen

O Ich habe starke, fast ständige Schmerzen

O Ich habe Anlaufschmerzen

O Meine Schmerzen sind nie belastungsabhängig

O Ich habe gelegentlich Schmerzen unter Belastung

O Ich habe kontinuierlich Schmerzen unter Belastung

O Ich habe Ruhe- oder Spontanschmerzen

\section{Gehhilfen:}


O Ich benötige keine Gehhilfe

O Ich benötige einen Stock als Gehhilfe

O Ich benötige eine Gehilfe mit Unterarmstütze

O Ich benötige einen Rollator als Gehhilfe

O Ich benutze einen Rollstuhl

\section{Allägliche Aktivität:}

O Ich bin nicht eingeschränkt und benötige keine Unterstützung in meinem Alltag

O Ich bin bei sportlicher Aktivität eingeschränkt, im Alltag jedoch nicht

O Ich bin im Alltag eingeschränkt

O Ich bin im Alltag stark eingeschränkt

Wenn Sie hierzu noch etwas anmerken möchten:

\section{Laufen/ Rennen:}

O Ich kann rennen, aber eingeschränkt

O Ich kann nicht rennen

O Ich kann rennen so lange ich will

O Ich kann ohne Einschränkung („stundenlang“) gehen

O Ich kann maximal: etwa 2 Std. (ca. 8-10 km) gehen

O Ich kann maximal: etwa 1 Std. (ca. 3-7 km) gehen

O Ich kann maximal: etwa 30 min (ca. 1-2 km) gehen

O Ich kann maximal: etwa 15 min (weniger als 1 km) gehen

O Ich kann nur in Etappen gehen, es sind immer wieder Pausen nötig

O Ich gehe nur noch bei mir zu Haus

O Ich gehe gar nicht mehr 


\section{Schmerzmitteleinnahme:}

O Ich nehme nie Schmerzmittel ein

O Ich nehme Schmerzmittel 1-2 Mal pro Woche ein

O Ich nehme Schmerzmittel 3-5 Mal pro Woche ein

O Ich nehme täglich Schmerzmittel ein

Welche Schmerzmittel nehmen sie, und in welcher Dosierung?

\section{Auf Zehenspitzen stehen:}

O Ich kann $10 \mathrm{Mal}$ hintereinander auf Zehenspitzen stehen

O Ich kann $5 \mathrm{Mal}$ hintereinander auf Zehenspitzen stehen

O Ich kann 1 Mal auf Zehenspitzen stehen

O Auf Zehenspitzen zu stehen ist nicht möglich

\section{Treppen steigen:}

O Normales Treppen steigen ist möglich

O Treppensteigen ist nur mit Geländer möglich

O Treppensteigen ist nur mit dem nicht betroffenen Fuß möglich

O Normales Treppensteigen ist nicht möglich

\section{Treppabwärtsgehen:}

O Normales Treppabwärtsgehen ist möglich

O Treppabwärtsgehen ist nur mit Geländer möglich

O Treppabwärtsgehen ist nur mit dem nicht betroffenen Fuß möglich

O Normales Treppabwärtsgehen ist nicht möglich 


\section{Gehprobleme:}

O Ich habe keine Gehprobleme auf welchem Untergrund auch immer

O Ich habe einige Gehprobleme auf unebenem Untergrund (z.B. Schotter)

O Ich habe erhebliche Gehprobleme auf unebenem Untergrund (z.B. Schotter)

O Ich habe leichte Probleme beim Besteigen von Leitern

O Ich habe erhebliche Probleme beim Besteigen von Leitern

Vielen Dank für Ihre Mitarbeit!

\subsection{Patienteneinwilligung}

Klinische Ergebnisse

nach Arthrodesen an oberem und unterem Sprunggelenk 


\section{- Einverständniserklärung -}

Patient:

Geburtsdatum:

Ich, wurde vollständig über Wesen, Bedeutung und Tragweite der klinischen Prüfung mit dem o.g. Titel aufgeklärt. Ich habe den Aufklärungstext gelesen und verstanden. Ich hatte die Möglichkeit Fragen zu stellen, und habe die Antworten verstanden und akzeptiere sie. Ich wurde über die mit der Teilnahme an der Studie verbundenen Risiken und dem möglichen Nutzen informiert.

Ich hatte ausreichend Zeit, mich zur Teilnahme an dieser Studie zu entscheiden und weiß, dass die Teilnahme an dieser wissenschaftlichen Studie freiwillig ist. Ich weiß, dass ich jederzeit und ohne Angaben von Gründen diese Zustimmung widerrufen kann, ohne dass sich dieser Entschluss nachteilig auf die spätere Behandlung durch meinen Arzt auswirken wird.

Mir ist bekannt, dass meine persönlichen Daten in verschlüsselter Form gespeichert werden. Eine Weitergabe dieser Daten an Dritte wird nicht erfolgen. Die Grundsätze der ärztlichen Schweigepflicht bleiben gewahrt.

I hre Daten werden am Ende der Akquise vollständig anonymisiert, so dass man später nicht zurückverfolgen kann, von wem die Daten sind. Bis zur Komplettierung der Datensätze werden die Datensätze pseudonymisiert. Dies bedeutet, dass die Daten mit einer Verschlüsselung ohne Namensnennung nur mit Nummern versehen 
werden. Die Zuordnung der Daten ist nur möglich, wenn hierfür der Schlüssel eingesetzt wird, mit dem die Daten pseudonymisiert wurden. Die Personen bezogenen Daten werden unter besonderen Schutzvorkehrungen getrennt von den pseudonymisierten Daten aufbewahrt. Eine Entschlüsselung ist nur durch den verantwortlichen Studienarzt möglich. Dritte erhalten keinen Einblick in die Originalunterlagen.

Ich habe eine Kopie der Patienteninformation erhalten. Ich erkläre hiermit meine freiwillige Teilnahme an dieser klinischen Studie.

Göttingen, den

Unterschrift des Patienten

Unterschrift des Arztes

/Doktorand

\section{Danksagung}

Mein ganz besonderer Dank gilt meinem Doktorvater, Herrn Prof. Dr. med. Klaus Dresing, leitender Oberarzt der Abteilung Unfallchirurgie, Plastische und Wiederherstellungschirurgie, für die Überlassung des Dissertationsthemas. Ich danke ihm besonders für die freundliche und 
geduldige Unterstützung bzw. Anleitung. Besonders bedanken möchte ich mich auch für die immer sehr zeitnahe Korrektur meiner Anfertigungen/ bzw. der endgültigen Arbeit.

Des Weiteren möchte ich mich bei den Mitarbeiter/innen der Abteilung für Medizinische Statistik der Universität Göttingen bedanken, die mich bei der Fertigstellung der Auswertung sehr unterstützt haben. Hervorheben möchte ich hierbei vor allem den wissenschaftlichen Mitarbeiter Herrn Dr. Klaus Jung sowie den Mathematikstudenten und Hilfswissenschaftler Herrn Tobias Lose.

Bedanken möchte ich mich ebenfalls bei den Mitarbeiter/innen des Zentralarchivs des Universitätsklinikums Göttingen, die bei der Herausgabe der Patientenakten stets zuvorkommend und freundlich waren.

\section{Lebenslauf}

Ich wurde am 15.03.1983 als Sohn des Zahnarztes Dr. med. dent. Peter Anger und seiner Ehefrau Susanne Anger, geborene Wehner, Physiotherapeutin, in Lübeck geboren. 
Von 1990 bis 1994 besuchte ich die Grundschule in Zarpen. Anschließend besuchte ich das Carl-Jacob-Burckhardt-Gymnasium in Lübeck von 1994 bis 1996.

Ab 1997 besuchte ich das Ostsee-Gymnasium Timmendorfer Strand, wo ich 2003 die allgemeine Hochschulreife erwarb.

Vom 01.10.2003 bis zum 30.06.2004 leistete ich meinen Wehrdienst beim

6. Panzerflugabwehrkanonenlehrbataillon in Lütjenburg ab.

Meine vorklinischen Krankenpflegepraktika leistete ich im August 2003 im Marienkrankenhaus in Lübeck, von Mitte Februar bis Mitte März 2005 in der Ostsee Praxis Klinik in Bad Schwartau und im September 2005 im Klinikum Neustadt ab.

Am 01.10.2004 begann ich das Studium der Humanmedizin an der GeorgAugust-Universität in Göttingen. Nach 4 vorklinischen Semestern bestand ich im September 2006 den ersten Abschnitt der ärztlichen Prüfung.

Zum Wintersemester 2006/07 setzte ich dann das Humanmedizinstudium in Göttingen mit dem klinischen Teil fort. Im Sommersemester 2007 begann ich dann die vorliegende Dissertation.

Meine klinischen Blockpraktika absolvierte ich an der Universitätsklinik Göttingen, sowie an den angeschlossenen Lehrkliniken und -Praxen.

In meinen Famulaturen wählte ich die Fächer Neurologie (Universitätsklinik Göttingen), Neurochirurgie (Universitätsklinik Göttingen), Unfallchirurgie (Universitätsklinik Göttingen), Mund-Kiefer-Gesichts-Chirurgie (Praxis Dr. Dr. Viehmann/ Bock in Lübeck) Allgemeinmedizin (Praxis Dr. med. F. Meisel in Lübeck) und Hals-Nasen-Ohren-Heilkunde (Universitätsklinik Göttingen). 
Im August 2009 begann ich mit der Ableistung des ersten Tertials des Praktischen Jahres in der Chirurgie des Weender Krankenhauses Göttingen. Seit Dezember absolviere ich mein zweites Tertial in der Hals-Nasen-OhrenHeilkunde der Universitätsklinik Göttingen.

Ab April 2010 werde ich mein letztes Tertial für Innere Medizin im Weender Krankenhaus in Göttingen verbringen. 